Analysis of Transient Plasmas for Pulsed Laser Deposition using Spatiotemporally Resolving Laser-Induced Fluorescence Spectroscopy 


\section{ANALYSIS OF TRANSIENT PLASMAS FOR PULSED LASER \\ DEPOSITION USING \\ SPATIOTEMPORALLY RESOLVING LASER-INDUCED FLUORESCENCE SPECTROSCOPY}

ANALYSE VAN VERANDERLIJKE PLASMA'S VOOR GEPULSTE LASERDEPOSITIE DOOR MIDDEL VAN RUIMTE- EN

TIJDSOPLOSSENDE LASER-GEÏNDUCEERDE FLUORESCENTIESPECTROSCOPIE 


\section{Ph.D. graduation committee:}

Chairman \& secretary

Prof. dr. ir. J.W.M. Hilgenkamp

\section{Promotors:}

Prof. dr. K.-J. Boller

Prof. dr. ing. A.J.H.M. Rijnders

Co-promotor:

Dr. ing. H.M.J. Bastiaens
University of Twente, The Netherlands

University of Twente, The Netherlands

University of Twente, The Netherlands

University of Twente, The Netherlands

\section{Members:}

Prof. S. Amoruso

Dr. ir. J. van Dijk

Prof. dr. J.L. Herek

Prof. dr. P.W.H. Pinkse
Università degli Studi di Napoli Federico II, Italy University of Technology Eindhoven, The Netherlands University of Twente, The Netherlands University of Twente, The Netherlands

Cover: Stylized photo of laser-induced fluorescence of yttrium in a plasma generated by ablating a $\mathrm{Y}_{2} \mathrm{O}_{3}$ target. The picture is taken in-plane with the excitation light sheet, which was tuned to an excitation wavelength of $Y$ at $294.85 \mathrm{~nm}$ (invisible in this picture). The excitation by the UV light results in fluorescence at several wavelengths between 535 and $565 \mathrm{~nm}$, visible in this picture as the thin green line through the plasma.

Copyright (c) K. Orsel (2016)

Analysis of Transient Plasmas for Pulsed Laser Deposition using Spatiotemporally Resolving Laser-Induced Fluorescence Spectroscopy

Ph.D. thesis, University of Twente, Enschede, The Netherlands

Illustrated - With references - With summary in English and Dutch

ISBN: 978-90-365-4052-0

DOI: http://dx.doi.org/10.3990/1.9789036540520

Printed by Gildeprint, Enschede, The Netherlands. 


\title{
ANALYSIS OF TRANSIENT PLASMAS FOR PULSED LASER \\ DEPOSITION USING \\ SPATIOTEMPORALLY RESOLVING LASER-INDUCED FLUORESCENCE SPECTROSCOPY
}

\section{DISSERTATION}

\author{
to obtain \\ the doctor's degree at the University of Twente, \\ on the authority of the rector magnificus, \\ prof. dr. H. Brinksma, \\ on account of the decision of the graduation committee, \\ to be publicly defended \\ on Thursday, Februari $11^{\text {th }}, 2016$ at 16:45
}

by

Kasper Orsel

born on December $11^{\text {th }}, 1984$

in Hengelo, Overijsel, The Netherlands 
This thesis has been approved by:

Promotors:

Prof. dr. K.-J. Boller

Co-promotor:

Prof. dr. ing. A.J.H.M. Rijnders

Dr. ing. H.M.J. Bastiaens

The work described in this thesis was performed at the Laser Physics and Non-linear Optics group, Department of Science and Technology, MESA+ Institute for Nanotechnology, University of Twente, P.O. Box 217, 7500 AE Enschede, The Netherlands. This research is supported by the Dutch Technology Foundation STW, which is part of the Netherlands Organization for Scientific Research (NWO) and partly funded by the Ministry of Economic Affairs (project number 10760). 
"Any sufficiently advanced technology is indistinguishable from magic."

- Arthur C. Clarke, Profiles of the Future, 1962

\section{To my family.}




\section{SUMMARY}

The goal of the work presented in this thesis is to realize a spectroscopic spatiotemporal mapping of selected species in laser-induced plasmas used in pulsed laser deposition (PLD), for a better understanding of the internal plasma dynamics and chemistry. We are especially interested in the influence of external parameters on the plasma dynamics and chemistry, such as the fluence of the ablation laser and the composition of the background gas and its pressure, as this can be the key to an improved understanding and control of stoichiometric film growth.

We have chosen a combination of laser-induced fluorescence (LIF) and absorption spectroscopy (AS). LIF enables the detection of plasma constituents even in "dark" plasmas, i.e., also when the plasma plume has cooled down and no longer spontaneously fluoresces. AS provides a means to calibrate the relative density distribution maps obtained from LIF measurements for obtaining absolute density distributions.

This research was carried out in parallel and in close collaboration with a second set of investigations performed by a colleague $\mathrm{PhD}$ student (Rik Groenen) in the IMS group (UT) who focused on investigating the properties of grown films, both during deposition with RHEED, as well as after film growth using X-ray diffraction measurements and atomic force microscopy.

The combination of spectroscopic (also called chemical) in-situ spatiotemporal mapping of individual plasma constituents with a detailed analysis of the grown films has enabled an unparalleled insight in the influence of the external PLD parameters on the plasma propagation, chemical evolution and material deposition. Having investigated different types of plasmas for growing different types of perovskites has shown that the choice of materials and background gas is of decisive influence for the growth of defect-free and stoichiometric thin films.

By applying two-dimensional and temporally resolved LIF, we have mapped, in space and time, the ground state populations of $\mathrm{Al}$ and $\mathrm{AlO}$ in plasma plumes generated by ablation of $\mathrm{LaAlO}_{3}$ (LAO) in both a pure $\mathrm{O}_{2}$ and a pure Ar background. Around a specific distance from the target, we observe a simultaneous vanishing of $\mathrm{Al}$ and appearance of $\mathrm{AlO}$ in an $\mathrm{O}_{2}$ background. This can be explained by the oxidation of $\mathrm{Al}$ in the background atmosphere occurring after a sufficient slowing of an expanding front of $\mathrm{Al}$ atoms. This is consistent with the observed absence of such effects when pure argon is taken as background. 
By applying two-dimensional LIF also on $\mathrm{Ti}$ and $\mathrm{TiO}$, we are able to directly link the oxidation of plasma species in $\mathrm{SrTiO}_{3}$ (STO) plasma for PLD to the stoichiometry and quality of the thin films grown. With spatiotemporal LIF mapping of the plasma species in various background gas compositions, we find that $\mathrm{Ti}$ and Sr have to be fully oxidized for a stoichiometric growth of crystalline thin films, which gives new input for modelling surface growth. The results show also that optical (LIF) monitoring might be applied for providing additional process control over the exact degree of stoichiometry of thin films.

For the third material investigated, we chose $\mathrm{YBiO}_{3}$ (YBO), a much less understood material compared to STO and LAO. YBO has piqued interest lately, but has only been successfully grown a few times using PLD, and also appears problematic with regards to epitaxial growth. By mapping Y, YO and Bi distributions in a YBO plasma, we can directly link the influence of oxygen present in the background gas during PLD on the oxidation of plasma species as well as the formation of stoichiometric perovskite films. With these particular materials, and in contrast to LAO and STO, we find that there is little direct chemical interaction taking place between the plasma plume constituents and the background gas. However, a strong influence of the background gas composition can still be seen on the YBO film growth, as well as a strong correlation between the oxygen fraction in the background gas and the amount of YO in the plasma plume. This observation is consistent with a plasma and growth dynamics where interaction takes place between the background gas and the target as well as the substrate, instead of a mixing and reaction of the plasma plume with the background gas, as observed in LAO and STO plasmas.

From the three materials we have investigated, it can already be seen that spatiotemporally resolved LIF is a highly valuable analytic approach for analyzing and understanding intricate details and features in PLD growth processes. Even when only mapping a select number of plasma constituents of a deposition material, much additional insight can be obtained. Having applied LIF in various different material systems, here, STO on LAO, STO on STO, and YBO on Lanthanum Strontium Aluminium Tantalum Oxide (LSAT), has clearly shown that there are no universal plasma and growth dynamics. Instead, each material system, due to its individual chemical and physical properties, provides its own dynamics in the plasma, both at the substrate and at the target. This observed wide span in variety promises that there is much more to discover and understand through LIF and possibly also other types of spectroscopy. Also, it might be attractive to consider installing LIF equipment as an additional standard monitoring tool, e.g., for achieving additional control in growth or for upscaling PLD growth in speed or area. 


\section{SAMENVATTING}

Het werk beschreven in dit proefschrift heeft als doel het in kaart brengen van specifieke bestandsdelen van laser-geïnduceerde plasma's, zoals gebruikt in gepulste laserdepositie (PLD), door middel van ruimte- en tijdsoplossende spectroscopie, voor een verbeterd begrip van de interne dynamiek en chemie van het plasma. In het bijzonder zijn we geïnteresseerd in de invloed van de externe PLD parameters op de plasma dynamiek en chemie, zoals de fluentie van de ablatie laser en de samenstelling en druk van het achtergrond gas, omdat deze een sleutelrol kunnen spelen in het verbeteren van het begrip van en controle over stoichiometrische filmgroei.

Wij hebben gekozen voor een combinatie van laser-geïnduceerde fluorescentie (LIF) en absorptiespectroscopie (AS). LIF stelt ons in staat om plasmabestandsdelen te detecteren in "donkere" plasma's, dat wil zeggen in plasma's die afgekoeld zijn en daardoor geen spontane fluorescentie meer uitzenden. AS geeft ons de mogelijkheid om de relatieve dichtheidsverdelingen die met LIF gemeten worden op een absolute schaal te kalibreren, wat leidt tot absolute dichtheidsverdelingen van de plasmabestandsdelen.

Dit onderzoek is in nauwe samenwerking uitgevoerd met Rik Groenen van de IMS groep (UT), wiens onderzoek gericht was op de eigenschappen van de gegroeide films, zowel tijdens als na afloop van de filmgroei.

De combinatie van het in-situ spectroscopisch (of chemisch) in kaart brengen van de plasmabestandsdelen met een gedetailleerde analyse van de gegroeide films zorgt voor een ongeëvenaard inzicht in de invloed van de externe PLD parameters op de plasma propagatie, chemische evolutie en materiaaldepositie. Uit het onderzoeken van verschillende plasmasamenstellingen voor het groeien van verschillende perovskiettypes is gebleken dat de keuze van materiaal en achtergrondgas van doorslaggevend belang is voor de groei van defectvrije en stoichiometrische dunne films.

Door het toepassen van tweedimensionale tijdsopgeloste LIF hebben we onderzoek gedaan naar de tijdsafhankelijke dichtheidsverdeling van $\mathrm{Al}$ en $\mathrm{AlO}$ in de grondtoestand in plasma pluimen die gegenereerd zijn door $\mathrm{LaAlO}_{3}$ (LAO) te ableren in zowel een puur $\mathrm{O}_{2}$ als een puur Ar achtergrondgas. Rond een specifieke afstand van het target nemen we weer dat het $\mathrm{Al}$ verdwijnt en tegelijkertijd AlO verschijnt in een $\mathrm{O}_{2}$ achtergrondgas. Dit kan verklaart worden door de oxidatie van $\mathrm{Al}$ in 
het achtergrond gas, nadat de $\mathrm{Al}$ atomen in het expanderende voorfront van de pluim voldoende vertraagd zijn. Deze verklaring wordt verder ondersteund door de afwezigheid van oxidatie van $\mathrm{Al}$ in een pure Ar achtergrond.

Door tweedimensionale LIF ook toe te passen op $\mathrm{Ti}$ en $\mathrm{TiO}$ kan een direct verband vastgesteld worden tussen de oxidatie van de plasmabestandsdelen van een $\mathrm{SrTiO}_{3}$ (STO) plasma en de stoichiometrie en kwaliteit van de gegroeide dunne films. Door het ruimte- en tijdsopgelost in kaart brengen van plasmabestandsdelen in verschillende samenstellingen van het achtergrondgas m.b.v. LIF, hebben we vastgesteld dat Ti en Sr volledig geoxideerd moeten zijn voor het stoichiometrisch groeien van kristallijne dunne films. Deze waarneming geeft nieuw inzicht voor het modelleren van de groei van dunne films aan kristallijne oppervlaktes. Deze resultaten laten zien dat optische analyse (d.m.v. LIF) van het depositieproces kan leiden tot een betere controle over de exacte stroichiomtrie van dunne films.

We hebben gekozen voor $\mathrm{YBiO}_{3}$ (YBO) als het derde materiaal, omdat dit materiaal veel minder onderzocht en begrepen is dan LAO en STO. Ondanks dat YBO nog weinig in PLD is gebruikt kent het materiaal een groeiende belangstelling. Bekend is dat YBO problematisch epitaxiaal te groeien is. Door de distributies van Y, YO en Bi te meten in een YBO plasma hebben we een direct verband gevonden tussen de aanwezigheid van zuurstof in het achtergrondgas tijdens PLD en de oxidatie van de plasmabestandsdelen en de vorming van dunne stoichiometrische perovskietfilms. In tegenstelling tot LAO en STO, zien we bij deze specifieke materialen zeer weinig directe chemische interactie tussen de plasmabestandsdelen en het achtergrondgas. Er is echter wel een sterke invloed waargenomen van de samenstelling van het achtergrondgas op de groei van YBO films. Bovendien is er een sterke correlatie tussen het zuurstofpercentage van het achtergrondgas en de hoeveelheid YO die aanwezig is in de plasma pluim. Deze obervaties komen overeen met plasmaen groeidynamieken waarbij het zuurstof in het achtergrondgas reageert met het target en het substraat, in plaats van directe chemische reacties tussen het achtergrondgas en de plasmapluim, zoals waargenomen in LAO en STO plasma's.

De drie materialen die onderzocht zijn laten duidelijk zien dat LIF een zeer waardevolle analytische methode is voor het onderzoeken en begrijpen van de complexe interacties en eigenschappen van het PLD groeiproces. Zelfs als slechts een selectie van de plasmabestandsdelen van een depositiemateriaal wordt gemeten, kan het inzicht in het proces sterk verhoogd worden. Het toepassen van LIF op verschillende materialen, in dit geval LAO gegroeid op STO, STO gegroeid op STO, en YBO gegroeid op Lantaan Strontium Aluminium Tantaal Zuurstof (LSAT), laat duidelijk zien dat er geen universele plasma- en groeidynamiek bestaan. Elk materiaal heeft zijn eigen unieke chemische eigenschappen en plasma dynamiek, zowel bij het target als het substraat. Deze grote verscheidenheid laat zien dat er nog zeer veel te ontdekken is d.m.v. LIF en mogelijk ook andere spectroscopische technieken. Ook is het aantrekkelijk om LIF systemen toe te voegen aan de standaard meetsystemen van PLD, bijvoorbeeld voor een verbeterde controle over de filmgroei, of voor het vergroten van het oppervlak of de snelheid van de filmgroei. 


\section{CONTENTS}

Summary vii

Samenvatting ix

1 Introduction 1

1.1 General aspects of spectroscopy . . . . . . . . . . . . . . . . . . 1

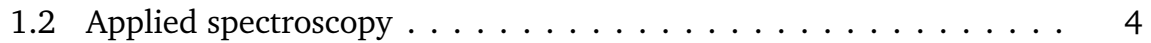

1.3 Spectroscopy on PLD plasmas . . . . . . . . . . . . . . 4

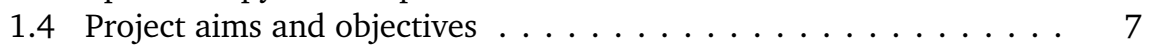

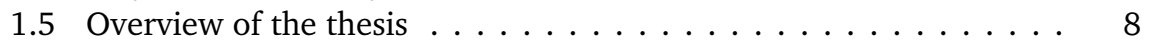

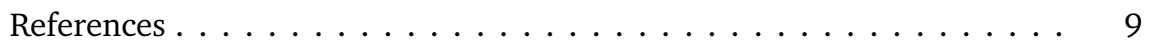

2 Theoretical aspects of Laser-Induced Fluorescence 11

2.1 Laser-Induced Fluorescence . . . . . . . . . . . . . . . . . . . . . . . . 12

2.2 Rate equations . . . . . . . . . . . . . . . . 13

2.2 .1 Two-level system . . . . . . . . . . . . . . . . . . . . 13

2.3 Absorption Spectroscopy . . . . . . . . . . . . . . . . . . . . . . . . . . . . . . . . . . . . . . .

2.4 Line broadening mechanisms . . . . . . . . . . . . . . . . . . . . 22

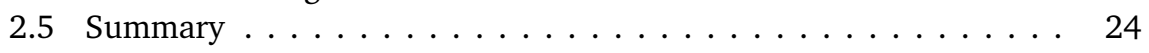

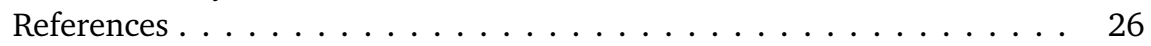

3 Experimental setup $\quad 27$

3.1 PLD chamber . . . . . . . . . . . . . . . . . . . . . . . . . . 28

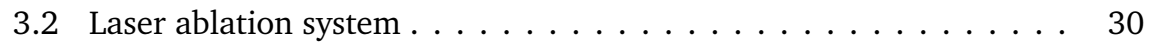

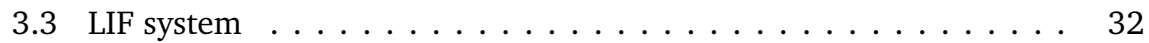

3.3 .1 Laser system . . . . . . . . . . . . . . . . . 32

3.3.2 Second harmonic generation ............. 34

3.3.3 Sheet formation and characterization . . . . . . . . . 36

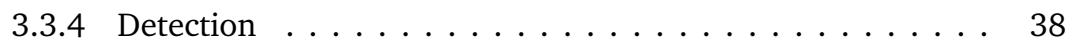

3.4 LIF measurements in saturation regime . . . . . . . . . . . . . . . . . 41

3.5 Absorption spectroscopy . . . . . . . . . . . . . . . . 45 
3.6 Synchronization . . . . . . . . . . . . . . . . . . . . . . . 49

3.7 summary . . . . . . . . . . . . . . . . . . . . . 51

References........................ 52

4 Spatial and temporal mapping of $\mathrm{Al}$ and $\mathrm{AlO}$ during oxidation in pulsed laser ablation of $\mathrm{LaAlO}_{3} \quad 53$

4.1 Experimental setup and methodology . . . . . . . . . . . . 54

4.2 Results . . . . . . . . . . . . . . . . . . . . 56

4.3 Discussion \& Conclusions . . . . . . . . . . . . . . . . 58

References . . . . . . . . . . . . . . . . . . . . . . 59

5 Influence of the oxidation state of $\mathrm{SrTiO}_{3}$ plasmas for stoichiometric growth of pulsed laser deposition films identified by laser-induced fluorescence

5.1 Experimental setup and methodology . . . . . . . . . . . 63

5.2 Results . . . . . . . . . . . . . . . . . . . . . . . . . 64

5.3 Discussion \& Conclusions . . . . . . . . . . . . . . 70

References . . . . . . . . . . . . . . . . . . . 72

6 Laser-induced fluorescence analysis of plasmas for stoichiometric growth of $\mathrm{YBiO}_{3}$ films with pulsed laser deposition 75

6.1 Experimental setup and methodology . . . . . . . . . . . 77

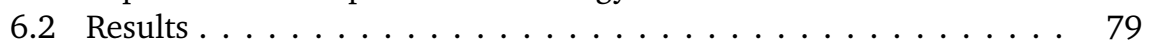

6.3 Discussion \& Conclusions . . . . . . . . . . . . . . . . . . . 84

References......................... 85

$\begin{array}{lll}7 & \text { Conclusions and discussion } & 89\end{array}$

List of Publications 95

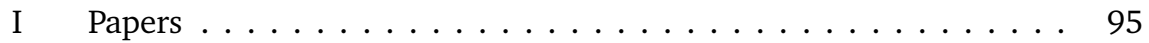

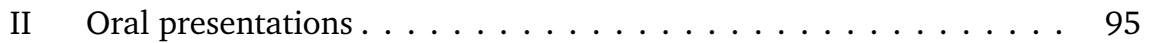

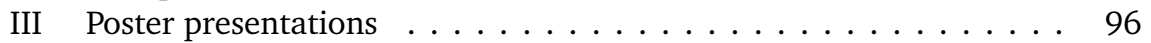

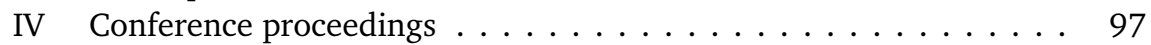

V Other publications .................. 97

$\begin{array}{ll}\text { Acknowledgments } & 99\end{array}$ 


\section{INTRODUCTION}

Optical spectroscopy is arguably one of the most flexible and broadly applicable experimental tools currently available to science, as it has enabled many fundamental discoveries about nature. One of the first optical spectroscopic experiments dates back as far as the early 19th century. In 1814, Joseph Fraunhofer published his research on the line-shaped gaps visible in the spectrum of the sun, though no clear explanation was found for this phenomenon at that time [1]. It would take another 45 years before Kirchhoff and Bunsen discovered that introducing specific elements to a flame results in the optical emission of wavelengths characteristic to the added elements [2]. They noticed that several emission lines coincided with the dark lines found by Fraunhofer, and correctly deduced that they were caused by absorption by atomic spectral lines of certain chemical elements present in the sun and earth atmosphere. These two discoveries later formed the basis for optical emission spectroscopy and absorption spectroscopy.

With these and many more examples, and via refining the method, optical spectroscopy became the key to the unraveling of the structure of atoms and molecules, and the discovery of wave mechanics, which later became quantum mechanics. Spectroscopy possesses a huge generality and universality through the large spectral range of electromagnetic (EM) waves that is available and due to well-known usually linear propagation properties of EM waves. Generally, any sample that contains charged elementary particles (e.g. electrons) may be analyzed which renders spectroscopy also of extremely high applicational relevance in a huge number of different situations.

\subsection{General aspects of spectroscopy}

Although spectroscopy is an extremely broadly applicable technique carried out in a large number of different experimental approaches, each of the approaches follows a general scheme as depicted in figure 1.1. Energy is added to a sample of 
interest, and electromagnetic radiation from the sample is analyzed. This scheme

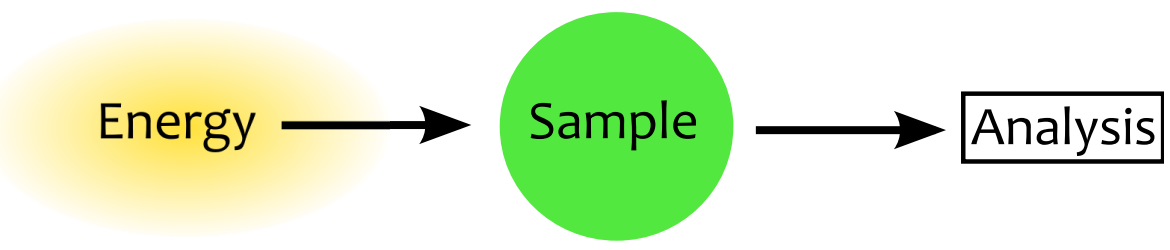

Figure 1.1: A generalized depiction of spectroscopy: energy is added to a sample of interest, and the electromagnetic radiation from the sample is analyzed.

is very general because each of the shown ingredients, energy supply, the nature of the sample and the way to analyze the emerging electromagnetic radiation, can take many different forms. Energy can be applied in various manners, for instance, as

- Electromagnetic radiation: In this case the excitation can be chosen in a highly specific manner. The reason is that the properties of the incident radiation can be controlled to great detail, such as its wavelength (e.g. X-rays, visible light or microwave radiation), its bandwidth (broadband like an incandescent light bulb or the sun, or narrowband as can be generated with lasers), its energy (from single photons to MegaJoules), its temporal structure (from continuous wave to attosecond pulses), its directionality (highly directional as can be generated with a laser or diffuse) and its polarization.

- Heat: Using thermal energy for the excitation of the sample is a much less specific way of excitation. In this case inelastic collisions between the particles with sufficient kinetic energy can lead to excitation of the constituents of the sample.

- Chemical energy. A certain degree in specificity of excitation may be obtained in chemical reactions with the sample. The products of this reaction may be left in certain excited states that relax back to the ground state through emission of light.

There are many other mechanisms for excitation, such as via mechanical or acoustic means (sonoluminescence [3]), via electron collisions in discharges or via beams of protons or other elementary particles.

The sample can be in any physical state, such as in the gas, liquid or solid state.

- Gas: Examples are exhaust gas from a combustion engine when investigating the amount of pollutants present [4], measuring the concentration of relevant species in the atmosphere [5] or in exhaled breath such as for medical applications[6].

- Liquid: When, e.g., searching for pathogens in biofluids [7] or detecting cancer cells in human blood [8]. 
- Solid: Spectroscopy on solids can help understand the fundamental structural properties of matter [9].

- Plasma: For example for investigation of the composition of interstellar plasmas.

In the analysis, EM radiation from the sample is detected by quantifying the properties of the radiation. Analysis can mean, e.g., simply measuring its power, its spectrum, the polarization, the directionality, or a combination of such properties.

To make this highly generalized explanation more accessible, let us look at the two examples given in the beginning of this section. In the case of Fraunhofers observations, the energy was provided by the sun in the form of spectrally broadband EM radiation. The sample was the atmospheres of both the sun and the earth through which the solar radiation had to propagate. Analysis was done using a prism after having selected a narrow range of propagation directions by means of a slit-shaped aperture, which allows to look at the power distribution as function of wavelength present in the solar spectrum. The typical spectrum as emitted by the sun in the visible range $(400-700 \mathrm{~nm})$ is depicted in figure $1.2 \mathrm{a}$. A representation of what Fraunhofer observed is shown in figure $1.2 \mathrm{~b}$. The dark lines present in this spectrum were later identified by Kirchhoff and Bunsen, who observed that some of these lines appear when thermally exciting certain chemical elements in the gas phase using a flame. If we put their experiment in terms of our general scheme of spectroscopy, energy was provided to their samples in the form of heat. The analysis of the emitted light and detection was done in a similar manner as by Fraunhofer. Figures $1.2 \mathrm{c}$ and $\mathrm{d}$ show the emission spectra of hydrogen and iron, respectively. It can be noticed that several emission lines of hydrogen in figure $1.2 \mathrm{c}$ overlap with absorption lines in figure $1.2 \mathrm{~b}$, but that there is basically no overlap with the emission lines of iron (figure 1.2d). The most basic conclusions from the example

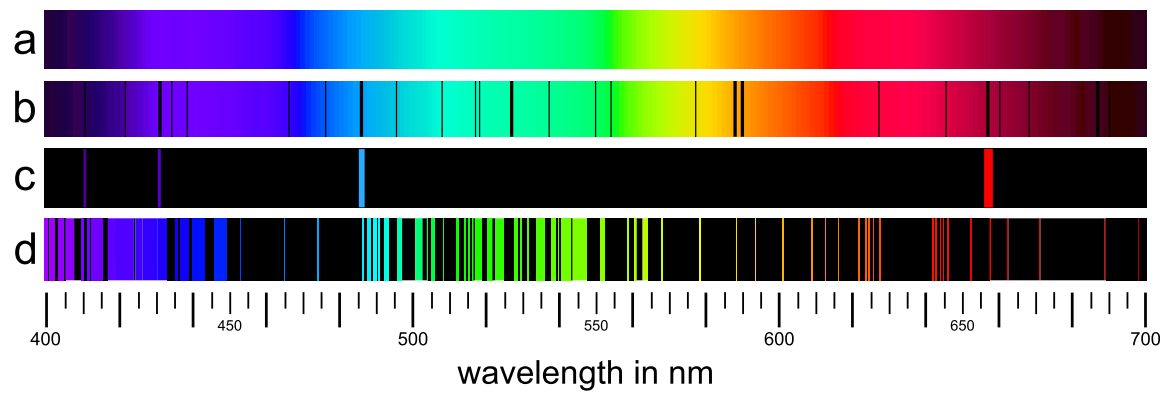

Figure 1.2: Spectra of (a) emission of the sun, (b) solar spectrum as observed on earth showning the Fraunhofer absorption lines [10], (c) emission lines of hydrogen [11] and (d) emission lines of iron [12].

spectra shown in figure 1.2 are prototypical for all other cases of spectroscopy. The comparison of spectra allows the deduction of the chemical composition of the sun 
and what elements are present in the sun and global atmospheres. These examples thereby demonstrate the important capability of spectroscopy to enable analysis of samples from a distance without having to physically probe it, even over millions of kilometers.

\subsection{Applied spectroscopy}

Given the named properties of spectroscopy, making use of spectroscopy, which is called applied spectroscopy, is one of the sharpest tools that are currently available in physics and other fields of research. The most important strengths of applied spectroscopy are that

- it can identify species. Particularly, spectroscopy allows to examine selectively only the species of interest, thereby providing what is called "chemical selectivity".

- it can measure quantitative concentrations. The levels of concentration that can be detected can actually be extremely low [13], making spectroscopy also one of the most sensitive tools in science.

- it can determine velocity and other environmental conditions. For instance, spectral lines are sensitive to velocity (Doppler shift), and thereby also to temperature (Doppler broadening). Also electric fields may be measured via, e.g., the Stark effect.

However, in order to actually reach these benefits in a given situation, considering the wide range of different spectroscopic techniques, it turns out that a careful selection of the specific approach has to be done. The goal in this selection is to provide a maximum of the most desired information with a technical approach that is also justified in terms of effort.

In this thesis we have selected laser-induced fluorescence (LIF) and absorption spectroscopy (AS). We have adapted these methods for providing chemical, temporal and spatial resolution in order to provide progress in spectroscopy for application in the analysis of laser generated plasmas, specifically the plasma in pulsed laser deposition (PLD). Striving for progress in spectroscopy of PLD is of particular promise, because PLD is a technique for the deposition of thin films that can exhibit exotic material properties, while at the same time not much is known about the detailed properties of the plasma that enables the growth.

\subsection{Spectroscopy on PLD plasmas}

A schematic depiction of the process of PLD is given in figure 1.3. In PLD, a substrate (on which material is to be deposited) and a target (made of the material to be deposited) are placed facing each other in a vacuum chamber. A high-power 
laser pulse is focused onto the target, creating a hot, dense plasma, thereby vaporizing a small amount of material. Due to rapid expansion, the plasma is ejected from the target in a highly forward-directed plume towards the substrate. Upon reaching the substrate, the plasma plume provides the material flux needed for film growth. When using lasers with a pulse length of several ns or longer, the ablation and deposition process can be described in five different stages, as depicted in figure 1.3. The stages are not completely separated and overlap somewhat in time [14].

In order to illustrate the high complexity of the plasma constituents, let us consider a simple and typical example for the choice of materials used in PLD, which is perovskites. This class of materials possesses an oxide structure of the form $\mathrm{ABO}_{3}$, where A and $\mathrm{B}$ are cations of different sizes (e.g. $\mathrm{SrTiO}_{3}, \mathrm{LaTiO}_{3}$ or $\mathrm{YBiO}_{3}$ ). The vacuum container used in PLD is typically containing a small and adjustable density of oxygen, with the goal to control the oxidation state of species in the plasma plume.

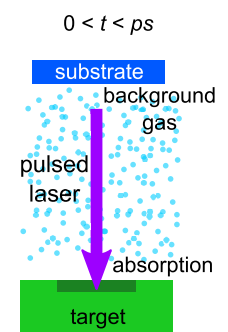

(1)

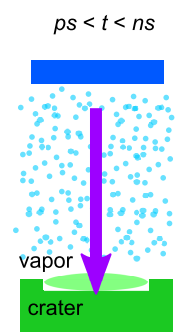

(2)

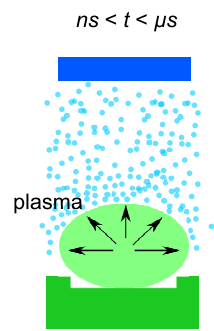

(3)

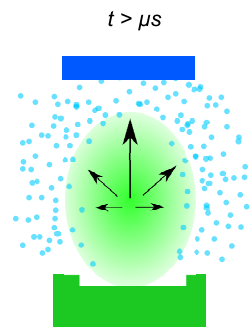

(4)

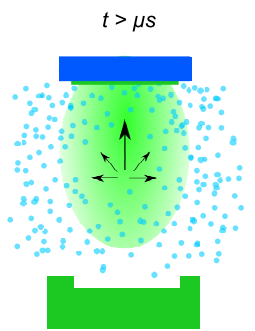

(5)

Figure 1.3: Schematic view of the stages of laser ablation and deposition: Light absorption by the target (stage 1), ejection of material from the target and subsequent ionization (stage 2), expansion of the plasma plume (stage 3), penetration of the plasma plume into a background gas (stage 4), and interaction of the plasma plume with the substrate (stage 5).

1. In the first stage, the laser light hits the target and is absorbed mainly by the electrons in the material. The electrons will transfer their energy to the atoms in the solid in a period of tens of ps, resulting in a strong heating of the illuminated volume. This stage is dominated by laser-solid interactions.

2. In the second stage, spanning the range of tens of ps to tens of ns, a thin layer of vapor is created by material ejected from the heated volume of the target. This vapor will continue to absorb energy from the laser, resulting in a strongly ionized plasma at the surface of the target, consisting of singly or multiply ionized target components, e.g. $\mathrm{A}^{+}, \mathrm{B}^{+}, \mathrm{O}^{+}, \mathrm{A}^{2+}$, etc. This stage is dominated by laser-gas or laser-plasma interactions.

3. After the laser pulse has ended, in the range between tens of ns and several $\mu \mathrm{s}$, the ablation enters the third stage, in which the plume expands adiabat- 
ically in three dimensions. Simultaneously, there will be chemical reactions of the plasma constituents. When target ablation takes place in vacuum, this is the final stage, and the shape, chemical and velocity distribution of the plasma plume will reach asymptotically constant values, expanding in a highly forward directed plume. In this stage, most of the ions in the plasma plume will have recombined with electrons from the plasma to become neutrals.

4. When target ablation takes place in an oxygen background, expansion will first occur as if in a vacuum, driven by the high plume pressure. However, after several $\mu \mathrm{s}$, the interaction of the plume constituents with the background gas will dominate the plume expansion. At this stage, the plasma will consist of ions $\left(\mathrm{A}^{+}, \mathrm{B}^{+}\right)$, atoms ( $\left.\mathrm{A}, \mathrm{B}, \mathrm{O}\right)$ and molecules ( $\mathrm{AO}, \mathrm{BO}, \mathrm{O}_{2}, \mathrm{AO}_{2}, \mathrm{BO}_{2}$, etc). All of these constituents are to be considered as highly dynamic, as the concentrations of these species are depending on the location in the plume and are expected to show rapid changes as well.

5. The plasma plume will reach the substrate after several $\mu \mathrm{s}$ in vacuum, or tens of $\mu \mathrm{s}$ in a background gas. The plasma constituents, including the oxygen from the background gas if present, will interact with the substrate, which leads to the growth of a thin film of material.

First demonstrated in 1965 [15], PLD has attracted much attention in the past two decades, boosted by the demonstration of PLD-grown high- $\mathrm{T}_{c}$ superconducting thin films [16]. It is now one of the preferred and technologically simple techniques for depositing a wide variety of materials with adjustable structural properties. Ranging from simple metals to complex multi-component single crystals, PLD has become a cornerstone of both material science and industrial thin film production [17].

Although many different materials are already being deposited on an industrial scale, growing complex films with atomic precision is still limited to small areas of approximately $1 \mathrm{~cm}^{2}$. One cause for this limitation is the complex and often unknown composition of the plasma plume, as indicated schematically in figure 1.3. Although material ablation can be stoichiometric in step (1) and (2), i.e., the ratio in which atomic species are present in the plasma just after target ablation are identical to the target, the ratio and chemical state in which they arrive at the substrate is not intrinsically stoichiometric. Material density, distribution, velocity and oxidation state vary during propagation of the plasma plume from target to substrate. These variations strongly influence the film growth quality. However, there is quite some lack of knowledge in PLD regarding these variations and what influence they have on the quality of grown films. What is required is an improved detection and tracking of the spatiotemporal dynamics and chemistry of the plasma plume.

The goal of the work done for this thesis is to provide such improved mapping for a better understanding of the dynamics and the chemistry taking place in the plasma plume during propagation from target to substrate. We are especially interested in the influence of external parameters on the plasma dynamics 
and chemistry, such as the fluence of the ablation laser and the composition of the background gas and its pressure, as we believe this is the key to an improved understanding and control of stoichiometric film growth.

\subsection{Project aims and objectives}

Having chosen plasmas that are used in PLD as the sample to be studied with spectroscopic means, there are several challenges ahead that are inherent to the properties of this sample. The plasmas generated in PLD are actually a most difficult and challenging spectroscopic target. The reasons are as follows:

- The plasma constituents undergo a rapid motion with high velocities (supersonic). Resolving the motion requires a spectroscopic method that is sufficiently fast. A solution to this is either using pulsed recording of the emitted light or a pulsed optical excitation or probing of the plasma.

- The plasma constituents perform a 3D motion in space over a range of typically 5 to $10 \mathrm{~cm}$. For resolving the 3D location of constituents the selected spectroscopic method has be able to offer a 3D distinguishable probing.

- The constituents are expected to undergo chemical reactions with each other and also with the background gas. To monitor this chemical conversion of species into each other, the spectroscopic equipment has to be selected for being flexible in terms of selectable wavelengths, in order to enable monitoring of various different species that are, however, chemically related.

- Finally, because the sample undergoes a wide range of temperatures, from elevated temperatures ( $\gg 10.000 \mathrm{~K})$ in a partially ionized state to lower temperatures $(\sim 1.000 \mathrm{~K})$, there is a first phase expected where the sample radiates via spontaneous emission (spontaneous fluorescence). This emission might be technically convenient to monitor, however, there are two problems. In the first phase, the excitation process is to a large extent unspecific due to the thermal nature of the excitation, which turns out to exclude quantitative measurements. In the later phase of plasma plume expansion and chemical development, which is actually of great direct relevance for the growth process, the problem is that the temperature is too low to excite spontaneous fluorescence such as would allow for spectroscopic detection.

The solution we have chosen here is a combination of laser-induced fluorescence (LIF) and absorption spectroscopy (AS). LIF enables detection of plasma constituents even when the plasma has cooled down and no longer spontaneously fluoresces. For accessing a wide range of materials that can be detected we have selected a dye laser combined with second harmonic generation that provides an extremely wide range of wavelengths for excitation $(250-900 \mathrm{~nm})$. The laser system also enables high chemical selectivity, due to a narrow spectral bandwidth 
for pre-selected excitation of atoms or molecular states, ensuring that only a single plasma constituent is detected at a time. Spatial resolution is provided by shaping the LIF excitation beams with special optics, allowing for mapping of thin cross-sections of the plasma plume. The short pulse length of the dye laser in the nanosecond range, in combination with a high-speed camera, provide an accordingly high temporal resolution.

This research was carried out in parallel and in close collaboration with a second set of investigations of PLD performed by another PhD student (Rik Groenen). In that part of the work, the properties of grown films are investigated, both during deposition with RHEED, as well as after film growth with X-ray diffraction spectroscopy and atomic force microscopy.

Linking plasma chemistry with film analysis has been done before, for instance by $\mathrm{Xu}$ [18], Wicklein [19] and Amoruso [20]. These measurements rely on spontaneous emission spectroscopy and can deliver valuable information as well. However, the analysis of spontaneous emission yields reliable results only in the early stages of plasma expansion when the plasma is still very hot. More detailed plasma analyses using LIF have also been done, but these lacked either film growth analysis (Dutouquet [21]), 3D spatial mapping of the plasma plume (Otis [22], Barsanti [23]) or absolute density measurements (Okada [24], Nakata [25]). Our combination of spatiotemporal mapping of the plasma constituents density and chemistry with detailed analysis of the grown films is what makes this research unique.

\subsection{Overview of the thesis}

This thesis consists of 7 chapters. Chapter one gives a brief introduction to applied spectroscopy and the motivations to implement laser-induced fluorescence and absorption spectroscopy on pulsed laser deposition plasmas. Chapter two recalls the relevant theoretical background needed to understand LIF and AS. Basic models and calculations are presented. Chapter three gives a description of the custom pulsed laser deposition setup into which we implemented both LIF and AS. The experimental challenges in setting up these diagnostics in a fully functional PLD chamber are discussed. Chapter four presents the results of the research on the spatial and temporal oxidation of Aluminium in a $\mathrm{LaAlO}_{3}$ plasma. Chapter five discusses the relation between the oxidation state of Titanium and Strontium and the stoichiometry of the grown film of $\mathrm{SrTiO}_{3}$. Chapter six presents the influence of the background gas composition on the nucleation rate of $\mathrm{YBiO}_{3}$ films. Chapter seven concludes this thesis by summarizing the results from this research, followed by suggestions for possible future work. 


\section{References}

${ }^{1}$ J. Fraunhofer, "Bestimmung des brechungs - und des farben-zerstreuungs - vermögens verschiedener glasarten, in bezug auf die vervollkommnung achromatischer fernrohre", Denkschriften der Königlichen Akademie der Wissenschaften zu München 5, 193 (1814).

${ }^{2} \mathrm{G}$. Kirchhoff and R. Bunsen, "Chemische analyse durch spectralbeobachtungen", Annalen der Physik 186, 161 (1860).

${ }^{3}$ S. Putterman and K. Weninger, "Sonoluminescence: how bubbles turn sound into light", Annual Review of Fluid Mechanics 32, 445 (2000).

${ }^{4}$ Q. Xia, H. Zuo, S. Li, Z. Wen, and Y. Li, "Remote passive sensing of aeroengine exhausts using ftir system", Spectroscopy and Spectral Analysis 29, 616 (2009).

${ }^{5}$ L. Nugent-Glandorf, F. Giorgetta, and S. Diddams, "Open-air, broad-bandwidth trace gas sensing with a mid-infrared optical frequency comb", Applied Physics B 119, 327 (2015).

${ }^{6} \mathrm{~T}$. Risby and F. Tittel, "Current status of midinfrared quantum and interband cascade lasers for clinical breath analysis", Optical Engineering 49, 111123 (2010).

${ }^{7}$ A. Bonifacio, S. Cervo, and V. Sergo, "Label-free surface-enhanced raman spectroscopy of biofluids: fundamental aspects and diagnostic applications", Analytical and Bioanalytical Chemistry 407, 8265 (2015).

${ }^{8}$ A. Pallaoro, M. Hoonejani, G. Braun, C. Meinhart, and M. Moskovits, "Rapid identification by surface-enhanced raman spectroscopy of cancer cells at low concentrations flowing in a microfluidic channel", ACS Nano 9, 4328 (2015).

${ }^{9}$ T. Luu, M. Garg, S. Y. Kruchinin, A. Moulet, M. Hassan, and E. Goulielmakis, "Extreme ultraviolet high-harmonic spectroscopy of solids", Nature 521, 498 (2015).

${ }^{10}$ https://en.wikipedia.org/wiki/File:Fraunhofer_lines.svg.

${ }^{11}$ https://en.wikipedia.org/wiki/File:Emission_spectrum-H.svg.

${ }^{12}$ https://en.wikipedia.org/wiki/File:Emission_spectrum-Fe.svg.

${ }^{13}$ A. Foltynowicz, T. Ban, P. Masłowski, F. Adler, and J. Ye, "Quantum-noise-limited optical frequency comb spectroscopy”, Physical Review Letters 107, 233002 (2011).

${ }^{14}$ C. Phipp, ed., Laser ablation and its applications, Optical Sciences (Springer Science and Business Media LLC, 233 Spring Street, New York, NY 10013, USA, 2007).

${ }^{15} \mathrm{H}$. Smith and A. Turner, "Vacuum deposited thin films using a ruby laser", Applied Optics 4, 147-148 (1965).

${ }^{16}$ D. Dijkkamp, T. Venkatesan, X. Wu, S. Shaheen, N. Jisrawi, Y. Min-lee, W. Mclean, and M. Croft, "Preparation of Y-Ba-Cu oxide superconductor thin films using pulsed laserevaporation from high Tc bulk material", Applied Physics Letters 51, 619-621 (1987). 
${ }^{17}$ R. Eason, ed., Pulsed laser deposition of thin films (John Wiley \& Sons, Inc., 2007).

${ }^{18}$ C. Xu, S. Wicklein, A. Sambri, S. Amoruso, M. Moors, and R. Dittmann, "Impact of the interplay between nonstoichiometry and kinetic energy of the plume species on the growth mode of $\mathrm{SrTiO}_{3}$ thin films", Journal of Physics D: Applied Physics 47, 034009 (2014).

${ }^{19}$ S. Wicklein, A. Sambri, S. Amoruso, X. Wang, R. Bruzzese, A. Koehl, and R. Dittmann, "Pulsed laser ablation of complex oxides: the role of congruent ablation and preferential scattering for the film stoichiometry", Applied Physics Letters 101, 131601 (2012).

${ }^{20}$ S. Amoruso, C. Aruta, P. Aurino, R. Bruzzese, X. Wang, F. M. Granozio, and U. S. di Uccio, "Oxygen background gas influence on pulsed laser deposition process of $\mathrm{LaAlO}_{3}$ and $\mathrm{LaGaO}_{3}$ ", Applied Surface Science 258, 9116 (2012).

${ }^{21}$ C. Dutouquet and J. Hermann, "Laser-induced fluorescence probing during pulsed-laser ablation for three-dimensional number density mapping of plasma species", Journal of Physics D: Applied Physics 34, 3356-3363 (2001).

${ }^{22} \mathrm{C}$. Otis and R. Dreyfus, "Laser ablation of $\mathrm{YBa}_{2} \mathrm{Cu}_{3} \mathrm{O}_{7-\delta}$ as probed by laserinduced fluorescence spectroscopy”, Physical Review Letters 67, 2102 (1991).

${ }^{23}$ S. Barsanti, M. Anwar-ul-Haq, and P. Bicchi, "Optical response and surface morphology of crystalline $\mathrm{Nd}^{3+}$-doped fluoride films grown on monocrystalline $\mathrm{LiYF}_{4}$ substrates by pulsed laser deposition”, Thin Solid Films 517, 2029 (2009).

${ }^{24}$ T. Okada and M. Maeda, "Laser spectroscopic studies of pulsed-laser deposition process for high- $\mathrm{T}_{c}$ thin films", Materials Science \& Engineering, B: Solid-State Materials for Advanced Technology 47, 64 (1997).

${ }^{25}$ Y. Nakata, T. Okada, M. Maeda, S. Higuchib, and K. Ueda, "Effect of oxidation dynamics on the film characteristics of Ce:YIG thin films deposited by pulsedlaser deposition", Optics and Lasers in Engineering 44, 147 (2006). 


\section{THEORETICAL ASPECTS OF LASER-INDUCED FLUORESCENCE}

\section{Introduction}

In this chapter, we will recall the basics of laser-induced fluorescence (LIF) and absorption spectroscopy (AS), because these two techniques were selected as the central spectroscopic approach and were implemented into the PLD setup for the characterization of the dynamics of the plasma plume and the monitoring of the chemical processes. Specifically, we will describe the theory that links the LIF to the relative densities of the species present in the PLD plasma. We also recall the basic properties of AS, and discuss how the combination of AS with LIF allows to calibrate the LIF detection system such that the absolute densities of the plasma constituents can be determined.

In section 2.1, we will discuss LIF in general as well as for the specific conditions under which this diagnostic technique can be applied to PLD plasmas in the present work. Most importantly, LIF allows the sensitive detection of atoms and molecules long after the plasma has been created. At that stage, the plasma has cooled down so far that it ceases to emit any spontaneous fluorescence (see section 1.4).

Setting up a simple set of rate equations for the population densities of a twolevel system in section 2.2, we identify two operating regimes for LIF depending on the laser intensity used for excitation. At sufficiently high laser intensity the LIF signal becomes independent of the laser intensity, called the saturation regime. This regime is most suitable for the determination of the relative densities of species. Further advantages of this regime are that the LIF signal is maximized, thereby providing the highest sensitivity in detection of species, and that detection becomes rather immune to spatial and temporal fluctuations of the excitation power and intensity [1].

At low laser intensity, we find that the LIF signal is linearly dependent on the 
laser intensity as well as on the species density. This regime is unsuitable for LIF, since spatial inhomogeneities in the excitation laser beam as well as shot-to-shot fluctuations of the intensity would have a strong influence on the LIF signal. However, this regime is very suitable for absorption spectroscopy, which we describe in section 2.3. AS is carried out at low laser intensities to ensure that the absorption scales linearly with the density of the species. Since AS is a technique in which the absorption of light passing through the plasma is spatially integrated, it is not sensitive to spatial inhomogeneities in the probe laser beam. We use AS in combination with LIF to determine the absolute spatial density distribution of species.

For both LIF and AS, knowledge about the spectral line shapes of the plasma constituents is important, as the validity of the approach discussed in this chapter places some requirements on the spectral shapes of both plasma species and excitation laser in terms of their spectral line width and dominant broadening mechanism. Therefore, in section 2.4 we will discuss the spectral line broadening mechanisms that are most common in PLD plasmas. We indicate which mechanisms are dominant and what influence they have on the evaluation of measured spectra and results.

\subsection{Laser-Induced Fluorescence}

To determine the presence of specific species in the PLD plasma and their relative density distribution we use LIF. The fluorescence of the species is induced through the absorption of laser photons, creating atoms and molecules in an excited state which can relax through spontaneous emission of photons.

LIF allows to monitor species in plasmas when the temperature has decreased below the point where excitation through collisions between the plasma constituents is possible. The bandwidth narrowing that can be achieved in most laser sources ensures that only a single specific transition is excited, providing a high chemical selectivity. Moreover, the high beam quality that can be achieved with a laser allows for a high spatial selectivity through focusing of the beam.

By using pulsed lasers generating sufficiently short pulses of a few nanoseconds duration, the temporal resolution with which the evolution of the plasma plume can be monitored is limited by the lifetime of the excited state, which for most of the strong atomic lines is in the order of several to tens of nanoseconds.

Most atoms and small molecules have electronic transitions in the UV and visible region. A most suitable source for light in this range is a tunable dye laser, which can generate light in the range of $400 \mathrm{~nm}$ to $900 \mathrm{~nm}$. Frequency doubling the output of the dye laser allows to generate wavelengths in the UV, as short as $200 \mathrm{~nm}$.

When an excited state decays, it can typically do so to several different lower levels, including the level from which it was excited. In the latter case, the fluorescence will be of the same wavelength as that of the excitation laser. For all other decay channels, the emitted fluorescence will have a longer wavelength, i.e., it is red-shifted with regard to the excitation laser. It is generally desirable to detect flu- 
orescence that is shifted in wavelength, since this allows the use of spectral filters to discriminate the LIF fluorescence from stray light originating from the excitation laser, such as reflected and scattered light at the entrance and exit windows of the PLD chamber (see section 3.1 for a detailed description of the PLD chamber).

In the next section, we will review the basic theory of laser excitation and fluorescence to obtain the relations between laser intensity, sample density and fluorescence power.

\subsection{Rate equations}

To illustrate the properties of LIF, we will analyze in this section the basic interaction of atoms and molecules with the laser radiation inducing the fluorescence. From this we will be able to relate the LIF signal to the laser intensity used for excitation, and the atomic and molecular densities in the plasma.

The simplest and most illustrative system to consider is a two-level system as depicted in figure 2.1. This system is qualitatively appropriate for most atomic species as well as simple molecules. In its simplest form the changes of the population in the two levels following the excitation by a laser source can be described with rate equations in terms of absorption, emission and quenching. Within the boundaries of this simple model, various basic concepts of LIF, including the linear relation between excitation and fluorescence at low excitation powers and saturation of the excited state when the excitation power is strongly increased, can be well understood.

\subsubsection{Two-level system}

For a two-level system in a laser radiation field, see figure 2.1, the population densities of the ground state $(i)$ and the excited state $(k)$ can be described using the rates for absorption, spontaneous and stimulated emission, and quenching, as indicated with arrows. Although atoms and molecules provide infinitely many states, as long as only a single excitation transition is used via frequency selective excitation, this model still properly describes the relation between the intensity of the laser used for excitation, the population of the excited state, and the amount of fluorescence from the system.

Figure 2.1 shows an atom or molecule with two energy levels $E_{i}$ and $E_{k}$ exposed to a radiation field. Black arrows represent the optically and collisionally induced transitions connecting the upper and lower levels. The rates for absorption and stimulated emission, $b_{i k}$ and $b_{k i}$, respectively, are related to the Einstein coefficient, $B$, by

$$
b=\frac{B I_{v}}{c}
$$

where $I_{v}$ is the incident laser intensity per unit frequency interval (spectral intensity, $W \cdot m^{-2} \cdot \mathrm{Hz}^{-1}$ ). $A_{k i}$ is the spontaneous emission rate given by the Einstein $A$ coefficient and $Q_{k i}$ is a non-radiative decay rate such as from the collisional 


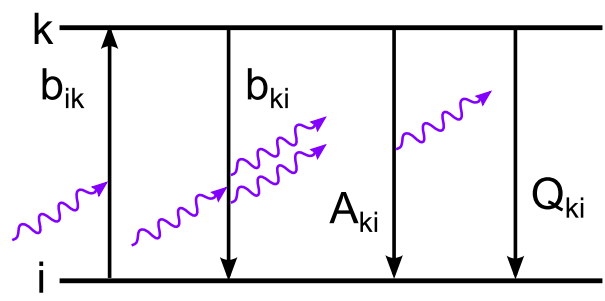

Figure 2.1: A simple two energy level diagram for modeling the rate equations of LIF. The purple arrows represent photons, the black arrows represent transitions between the levels.

quenching $[1,2] . B_{i k}, B_{k i}$ and $A_{k i}$ are related by

$$
\begin{gathered}
B_{k i}=\frac{A_{k i} c^{3}}{8 \pi h v^{3}} \\
g_{i} B_{i k}=g_{k} B_{k i}
\end{gathered}
$$

where $g_{i}$ and $g_{k}$ are the degeneracies of the respective states. The temporal derivatives of the state population, denoted by $N_{i, k}$, can be written as

$$
\begin{aligned}
& \frac{d N_{i}}{d t}=\dot{N}_{i}=-N_{i} b_{i k}+N_{k}\left(b_{k i}+A_{k i}+Q_{k i}\right) \\
& \frac{d N_{k}}{d t}=\dot{N}_{k}=N_{k} b_{i k}-N_{k}\left(b_{k i}+A_{k i}+Q_{k i}\right) .
\end{aligned}
$$

As this is a closed two-level system, this assumes that there is no significant ionization or excitation to other states, such that the total state population does not change:

$$
N_{i}^{0}=N_{i}+N_{k} .
$$

Here, $N_{i}^{0}$ is the ground level population prior to laser excitation. In the following, we derive the steady-state behavior of the two-level system to obtain its dependence on the laser spectral intensity. We will assume that the laser linewidth has the same shape and size as the absorption linewidth, by which we neglect spectral hole burning. Furthermore, we assume that the excitation intensity does not change too rapidly vs. time, such that ultimately a steady state is established among all the processes depicted in figure 2.1, i.e., that ultimately level populations become constant vs. time $\left(\dot{N}_{i}\right.$ and $\left.\dot{N}_{k}=0\right)$. Temporally integrating equation 2.5 for the population of the excited state $\left(N_{k}\right)$ by eliminating the population of the ground state $\left(N_{i}\right)$ via equation 2.6 yields:

$$
N_{k}(t)=\frac{b_{i k} N_{i}^{0}}{2}\left(1-e^{-r t}\right),
$$

where $r=b_{i k}+b_{k i}+A_{k i}+Q_{k i}$ and where we have assumed that the excited 
state is initially not populated $\left(N_{k}(t=0)=0\right)$. The latter holds true for most electronic states accessible via transitions in the UV and the visible regions in PLD plasmas, when the plasma plume has expanded and cooled down to temperatures in the order of $5.000 \mathrm{~K}$, which is typically after about $10 \mu \mathrm{s}$ after the ablation of the target. When the laser excitation starts, for $r t \ll 1$, the upper level population builds up linearly with time,

$$
N_{k}(t)=b_{i k} N_{i}^{0} t
$$

and then saturates at a steady-state value of

$$
N_{k}=\frac{b_{i k} N_{i}^{0}}{r}
$$

for $r t \gg 1$. For typical experimental values, as described in section 3.4, values for $r$ are in the order of $10^{11}$ to $10^{12}$, which means a steady state $(r t \gg 1)$ is reached within the first tens of ns of the excitation pulse. Assuming that a steady state is achieved, equation 2.9 describing the population of the excited state can be rewritten as

$$
N_{2}=N_{1}^{0} \frac{b_{i k}}{b_{i k}+b_{k i}} \frac{1}{1+\frac{A_{k i}+Q_{k i}}{b_{i k}+b_{k i}}}=N_{i}^{0} \frac{B_{i k}}{B_{i k}+B_{k i}} \frac{1}{1+\frac{I_{s a t}^{v}}{I_{v}}}
$$

where the saturation spectral intensity $I_{\text {sat }}^{v}$ is defined as

$$
I_{s a t}^{v} \equiv \frac{\left(A_{k i}+Q_{k i}\right) c}{B_{i k}+B_{k i}} .
$$

These equations can be used to relate the number of fluorescence photons that

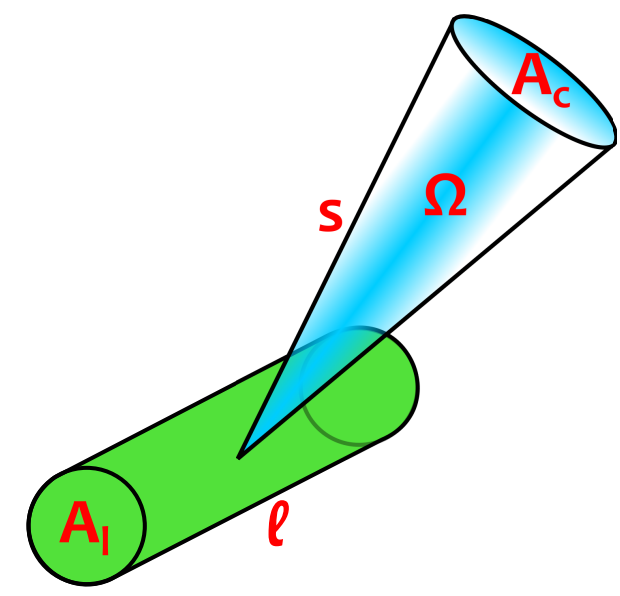

Figure 2.2: Detection of fluorescence from a volume of $A_{l} \times \ell$ with a solid angle $\Omega$. 
will be detected as a function of time to the density of atoms $N_{i}^{0}$ present in a probed volume. We define the following parameters: the spotsize of the excitation laser $A_{l}$, in a column of excited PLD plasma of length $\ell$ (see figure 2.2), where the fluorescence photons are captured by a detector with an aperture $A_{c}$ at a distance $s$.

The fluorescence power $F$ at the detector can then be expressed as

$$
F=\eta h v N_{2} A_{k i} \frac{\Omega}{4 \pi} \ell A_{c}=\eta h v \frac{\Omega}{4 \pi} \ell A_{c} N_{1}^{0} \frac{B_{i k}}{B_{i k}+B_{k i}} \frac{A_{k i}}{1+\frac{I_{s a t}^{v}}{I_{v}}}
$$

where $\eta$ is the quantum efficiency of the detector, $h v$ is the photon energy, $h$ is Planck's constant, and $v$ is the frequency of the emitted fluorescence. For simplicity we define a constant $C_{i k}$, comprising all physical detection parameters:

$$
\begin{gathered}
C \equiv \eta h v \frac{\Omega}{4 \pi} \ell A_{c} \\
F=C N_{1}^{0} \frac{B_{i k}}{B_{i k}+B_{k i}} \frac{A_{k i}}{1+\frac{I_{s a t}^{v}}{I_{v}}} .
\end{gathered}
$$

The main variable in equation 2.14 that can be easily changed during LIF experiments is the laser excitation spectral intensity, $I_{v}$. We will now examine the behavior of the fluorescence for low as well as high laser intensities.

Linear regime When the laser excitation intensity is low, much lower than the saturation intensity $\left(I_{v} \ll I_{\text {sat }}^{v}\right.$ ), equation 2.14 can be reduced to

$$
F=C N_{i}^{0} \frac{B_{i k}}{B_{i k}+B_{k i}} \frac{A_{k i}}{I_{s a t}^{v}}=C N_{i}^{0} B_{i k} I_{v} \frac{A_{k i}}{A_{k i}+Q_{k i}} .
$$

This equation shows that if we assume that the quenching $Q_{k i}$ does not change with time, the fluorescence signal power $F$ is proportional to both the excitation intensity and the density. This is referred to as the linear regime of a two-level system, as depicted in figure 2.3. The linear regime is important for our research on the absolute densities of PLD plasma constituents. We will use AS for the absolute calibration of LIF, in which case AS must be carried out in the linear regime (see section 2.3).

Saturation regime For a laser intensity much larger than the saturation intensity $\left(I_{v} \gg I_{\text {sat }}^{v}\right)$, equation 2.14 reduces to

$$
F=C N_{i}^{0} \frac{B_{i k}}{B_{i k}+B_{k i}} A_{k i}
$$

At this point the population of the lower level becomes depleted, and the fluorescence signal is no longer dependent on either the laser intensity or the quenching. 


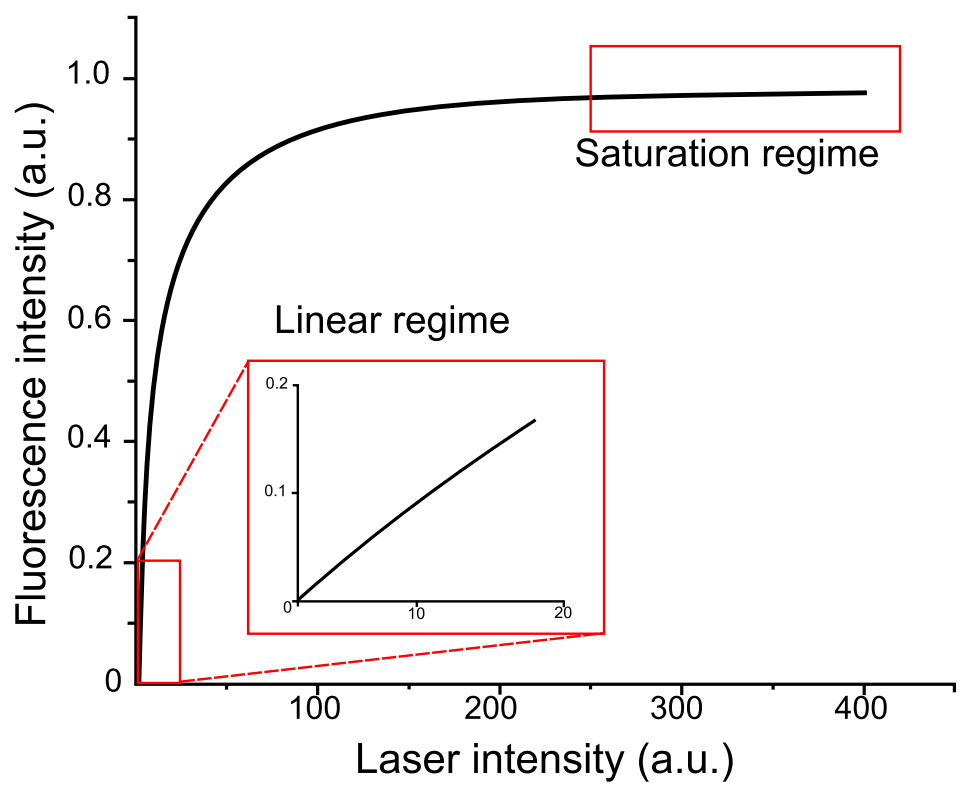

Figure 2.3: Calculated laser-induced fluorescence intensity of a two-level system as function of excitation laser power. At low laser intensity, the fluorescence intensity is linearly dependent on the laser intensity. At higher power, the excited state becomes saturated and the fluorescence intensity becomes independent of the laser intensity.

For such high laser intensities, the population difference between ground state and excited state is named saturated, which means that every atom that relaxes to the ground state will immediately become excited again. The saturation regime is important when spatially mapping the plasma plume using LIF, because small spatial inhomogeneities, which are always present in the excitation beam, as well as shot-to-shot fluctuations of the output power and thus excitation intensity of the laser will have only little effect on the induced fluorescence intensity (see figure 2.3). Another advantage for spatial mapping in the saturation regime, observing that the rhs. of equation 2.16 is always bigger than that of equation 2.15 , is that it provides the highest possible LIF signal. It should be noted that the formulas discussed in the previous section assume an excitation laser beam with a homogeneous spatial intensity, as shown in figure 2.2. However, most laser beams have a Gaussian spatial intensity distribution. In this case, saturation is not achieved in the edges of the laser beam. The consequences of this effect for data evaluation, are discussed in more detail in section 3.4.

Figure 2.4 illustrates schematically the geometrical arrangement that was used for mapping the density of plasma constituents using LIF. A thin sheet of light, formed from a laser beam, is exciting the atoms in the intersection volume formed with the plume volume. The fluorescence from this intersection is imaged onto a camera. When operating in the saturation regime, equation 2.16 shows that 


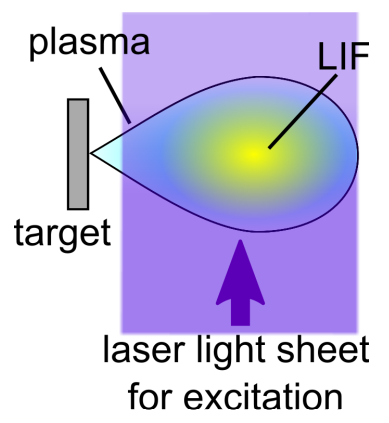

Figure 2.4: Mapping of the relative density of constituents of a PLD plasma plume using LIF. The laser beam providing the excitation is formed into a thin light sheet selectively inducing fluorescence in one of the plasma species. The fluorescence from the intersection of the laser light sheet with the plasma (in yellow) is observed from the top with a camera (iCCD).

the fluorescence signal $F$ is proportional to ground state density $N_{i}$. However, to retrieve also absolute values for the distribution of $N_{i}$, as equation 2.12 shows, several external parameters, such as the solid angle of the detection system, need to be determined in absolute terms. Specifically, the camera converts the fluorescence signal into an electronic signal, which it does with a certain efficiency, $\eta$, that is not precisely known. Also, the use of a lens system and possibly optical filters introduces a certain loss to the fluorescence intensity due to reflection and absorption, both of which are wavelength dependent and difficult to determine in absolute terms. Finally, atomic or molecular parameters might not be known well. Therefore, in practice it is required to define a calibration factor, $C$, that incorporates all unknown factors and wavelength dependencies in the relation between the relative density $N_{r e l}$ measured by the camera in the form of a LIF intensity distribution and the absolute density $N_{i}\left(C \cdot N_{r e l}=N_{i}\right)$. To determine the calibration factor of the detection system, $C$, it is possible to apply absorption spectroscopy (AS) with a nearly identical excitation and detection setup, as will be described in the next section.

\subsection{Absorption Spectroscopy}

LIF can be used to determine the relative spatial density distribution of species in the plasma plume as described in the preceding section. However, because it is difficult to retrieve from LIF also information on the absolute density, an additional technique is required to provide the missing information. Several methods are available to calibrate LIF to provide absolute densities, for instance calibration by parts, where all optical components are calibrated individually, which is tedious and introduces undesired errors. Other methods are to detect either Rayleigh scattering [3] or LIF from a well known "calibration gas" provided at known absolute 
densities. However, these measurements would have to be done across a wide range of wavelengths to provide an accurate spectral calibration. The method that we have chosen for providing an absolute calibration of LIF is absorption spectroscopy (AS). Similar combinations of LIF and AS have previously been applied in plasma research $[4,5]$. In this section, we will discuss AS that provides line integrated densities of species, yet, without any information on the spatial distribution of the density. We will show that by combining the results obtained from LIF and AS measurements, we are able to obtain absolute spatial density distributions of the species in the plasma plume. Figure 2.5 shows the geometrical arrange-

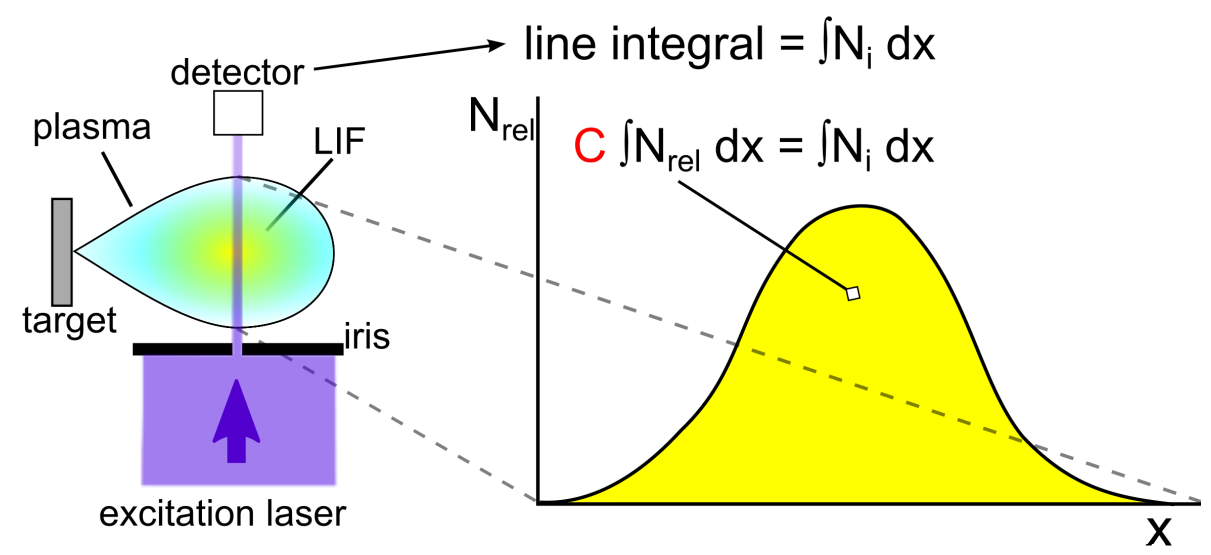

Figure 2.5: Schematic showing the combination of LIF and AS in a PLD plasma to determine an absolute density map of the constituents. For AS only a small section of the laser light sheet is sent through the plasma plume, as shown on the left hand side. Along the beam path, LIF will provide the relative density distribution $N_{\text {rel }}$, as shown in the graph on the right. Measuring the absorption provides the line integrated density of particles in the laser beam, which equals the integral of the relative density distribution along the beam path. By equating both integrals, the calibration factor $C$ for the detection system can be determined.

ments used for AS. On the lhs, the plasma plume is depicted throughout which the relative density distribution within a thin excitation laser light sheet has been determined by LIF. The density distribution $N_{r e l}$ along a small section of that sheet (as schematically defined by an iris) is shown on the rhs along the beam path ( $x$-axis). Measuring the absorption along the same beam path, AS provides an attenuation, that is, at sufficiently low densities, proportional to the line integral of the density of particles $\int N_{i} d x$. In brief, equating this line integral to the relative distribution, $\int N_{\text {rel }} d x$, provides the calibration factor $C$ for the LIF measurement:

$$
C \int N_{r e l} d x=\int N_{i} d x
$$


In the following paragraph, we will discuss the basic principles of AS necessary to calculate the line integral of the density of particles from an absorption spectrum.

The intensity $I$ of the laser light sheet, along the propagation path through the plasma plume ( $x$-direction), decreases due to absorption over distance $d x$ by

$$
d I_{v}=-\alpha_{i k}(v) I_{v} d x
$$

At a given light frequency, $v$, the absorption coefficient $\alpha_{i k}(v)$ for a transition from state $i$ to state $k$ is dependent on the population densities of the lower $\left(N_{i}\right)$ and upper $\left(N_{k}\right)$ levels, and on the optical absorption cross section $\sigma_{i k}(v)$ of each atom:

$$
\alpha_{i k}(v)=\sigma_{i k}(v)\left[N_{i}-\left(g_{i} / g_{k}\right) N_{k}\right]
$$

The spectrally integrated absorption cross-section is related to the spontaneous emission rate, $A_{k i}$, by [6]

$$
\int \sigma_{i k}(v) d v=\frac{g_{i}}{g_{k}} \frac{c^{2}}{8 \pi v^{2}} A_{k i} .
$$

Equation 2.19 reduces to $\alpha_{i k}=\sigma_{i k}(v) N_{i}$ if the population density of the excited state is much lower than the ground state density $\left(N_{k} \ll N_{i}\right)$. This is fulfilled for atoms in PLD plasmas under the experimental circumstances used in this thesis, e.g., a background gas pressure of $0.1 \mathrm{mbar}$ and a delay time between plasma creation and measurement of $>10 \mu \mathrm{s}$, in which case the plasma has cooled down to the point where thermal excitation of atoms in the plasma is small. For sufficiently small intensities $I_{v}$, the excitation rate is small compared to the decay rate into the ground state, $i$. At this point, the population density $N_{i}$ does not depend on the intensity $I_{0}$ and the absorption scales linearly with the density of absorbing atoms. Integration of equation 2.18 in this linear regime yields Beer's law of absorption [7]

$$
I_{v}=I_{v, 0} e^{-\alpha(v) x}=I_{v, 0} e^{-\sigma_{i k}(v) N_{i} x} .
$$

To more easily relate this expression to the measurements, i.e., the amount of light absorbed at a certain wavelength, to the density of particles $N_{i}$, we now define the optical depth $\tau(v)$ as

$$
\tau(v) \equiv \ln \left(\frac{I_{v, 0}}{I_{v}}\right)=\sigma_{i k}(v) N_{i} x .
$$

We also make use of the dimensionless absorption oscillator strength $f_{i k}$, which gives the ratio of the power absorbed by an atom or molecule on the transition $i \rightarrow k$ to the power absorbed by a classical Lorentz oscillator on its eigenfrequency $v_{i k}$ :

$$
f_{i k}=\frac{g_{i}}{g_{k}} \frac{m_{e} \epsilon_{0} c^{3}}{2 \pi e^{2} v_{i k}^{2}} A_{k i} .
$$


Rewriting equation 2.20 in terms of $f_{i k}$, one obtains

$$
\int \sigma_{i k}(v) d v=\frac{e^{2}}{4 \epsilon_{0} m c} f_{i k}=\pi r_{0} c f_{i k},
$$

where $r_{0}$ is the classical electron radius defined as

$$
r_{0}=\frac{e^{2}}{4 \pi \epsilon_{0} m c^{2}} .
$$

Making use of the optical depth in equation 2.24 yields

$$
\int \tau(v) d v=\pi r_{0} c f_{i k} N_{i} x,
$$

which relates the spectrally integrated optical depth (integrated over the absorption line width) to the absorption oscillator strength and the distance $x$ traveled through a medium of spatially constant density $N_{i}$ (homogeneously distributed medium). For inhomogeneously distributed media, as are of interest here, this expression can be generalized as

$$
\int \tau(v) d v=\pi r_{0} c f_{i k} \int N_{i}(x) d x
$$

or

$$
\int N_{i}(x) d x=\frac{\int \tau(v) d v}{\pi r_{0} c f_{i k}}
$$

where $\int N_{i}(x) d x$ is the density of particles, integrated along the beam path. From equation 2.28 it can be seen that the line integrated density of particles can be obtained from a measurement of the spectrally integrated optical depth. Equating this to the integral of the density distribution according to equation 2.17 one obtains the calibration factor $C$.

When using the method described in this section, it is important to determine what spectral line broadening mechanisms are dominant during the measurements. Some broadening mechanisms, such as Stark broadening, change not only the spectral line shape but also the spectral integral of the absorption cross-section. Equation 2.28 can be corrected to take this change into account. Another complication can be that PLD plasmas are highly transient and comprise large temperature and density gradients, which means that the influence of broadening mechanisms can be inhomogeneously distributed across the plasma plume, making them very hard to correct for. It is therefore greatly preferable to operate AS in conditions in which the dominant line broadening mechanisms have no effect on the spectrally integrated absorption cross-section. In the next section we will discuss the most common broadening mechanisms in plasmas, evaluate their respective relative influence on the spectral line broadening to deduce the dominant mechanism and deduce whether the absorption cross-section is influenced for the PLD plasmas 
investigated in this thesis.

\subsection{Line broadening mechanisms}

Although an atomic transition is associated with a well-defined amount of energy, absorption or emission spectra are never truly monochromatic but somewhat broadened. The broadening of spectral lines is described with a line profile of a specific shape that is determined by a combination of intrinsic characteristics, such as the lifetime of the state, and external parameters, such as temperature and pressure. Loosely, spectral lines can be characterized by the center wavelength, the peak value (e.g., the absorption or emission strength at line center) and the full-width-at-half-maximum (FWHM) spectral bandwidth.

In this section, we recall and discuss the strength of the broadening mechanisms that are typically present in PLD plasmas. We will show that in PLD plasmas Doppler broadening is dominant and calculate that several microseconds after ablation the line width typically amounts to several picometers. As Doppler broadening only changes the absorption wavelength of the individual particles but does not affect the spectrally integrated absorption rate, AS as discussed for the derivation of equation 2.28 can be applied to a plasma where Doppler broadening is the dominant broadening mechanism.

Doppler broadening Due to thermal motion, the individual atoms and molecules in a sample show slightly Doppler shifted absorption or emission wavelengths. When looking at a sample containing many particles, the sum of contributions of the individual particles will lead to a so-called Doppler broadening of the spectral line with a FWHM of [8]

$$
\delta v_{D}=\left(\frac{4 \sqrt{\ln 2}}{c}\right) v_{0} \sqrt{\frac{2 R T}{M}},
$$

where $T$ is the sample temperature, $v_{0}$ is the light frequency at the line maximum, $R$ is the gas constant and $M$ is the molar mass. To give an example of the amount of Doppler broadening in a PLD plasma, let us consider the $294.2 \mathrm{~nm}$ line of Ti at $5.000 \mathrm{~K}$, which is a typical temperature when the plasma plume has expanded by several centimeters. The Ti line in this case would have a Doppler broadening of $2.13 \mathrm{pm}$, corresponding to $7.46 \mathrm{GHz}$.

Pressure broadening When the emitting species interact through elastic collisions with other particles, for instance the background gas, their energy levels will shift and thereby broaden slightly due to interaction of the electron clouds. Pressure broadening is mostly dependent on the temperature (which influences the collision rate), the pressure, and the weight of the colliding particles. A higher pressure and thus higher particle density and temperature will lead to a higher collision frequency. This is also true for a higher temperature, since the particles 
are moving at a higher speed. Lighter particles will be more strongly influenced by a collision than heavier particles. Therefore, to calculate the strongest influence pressure broadening could have, we look at the lightest particles present in the plasmas investigated in this thesis, which are aluminium atoms, and consider collisions at the highest pressure ( $0.1 \mathrm{mbar})$ and at a temperature typical for times more than $10 \mu$ s after ablation $(5.000 \mathrm{~K})$. The pressure broadening in this case amounts to less than $0.1 \mathrm{pm}$ [7], making its contribution negligible compared to Doppler broadening. Even at a temperature of $50.000 \mathrm{~K}$, which may be reached in the first few microseconds after ablation, Doppler broadening would still be dominant over pressure broadening.

Resonance broadening Resonance broadening is caused by optical interaction between an excited atom and an identical atom in the ground state when the excited level and the ground state are connected by a strong allowed electric dipole transition, usually a resonance line. The broadening is approximately proportional to the density of the atoms and the oscillator strength $f_{i k}$ of the transition. As an example of one of the highest oscillator strengths, let us look at the $553 \mathrm{~nm}$ line of barium $\left(f_{i k}=1.64\right)$. At a particle density of $1.5 \times 10^{17} \mathrm{~cm}^{-3}$, the resonance broadening would have a FWHM of approximately $30 \mathrm{pm}$. However, such densities only occur in the first few microseconds of the plasma expansion, when the plume has a volume of only a few cubic millimeters. At longer delay times after target ablation $\left(>10 \mu \mathrm{s}\right.$ ), densities are typically much lower, below the order of $10^{14} \mathrm{~cm}^{-3}$, resulting in a broadening in the order of $0.1 \mathrm{pm}$ or less [9], thus having no significant effect on the total line broadening.

Stark broadening Collisions with charged particles, such as electrons and ions, can cause a Stark shift of the energy levels of emitting atoms and molecules. This Stark effect results in a broadening of the emission lines as well as a shift of the linecenter wavelengths. The electron density of a plasma, which is one of the primary variables in determining the degree of the Stark broadening, is however largely unknown for PLD plasmas, especially at longer delay times after target ablation. The reason for this lack of knowledge is that the most common tool for determining the electron density in a plasma is to calculate it from the spectral width of the Stark broadened line profile of the plasma constituents. Unfortunately, such a calculation is only justified if the plasma is in so-called local thermodynamic equilibrium, which is only the case if the plasma has a sufficiently high electron density of at least $10^{15} \mathrm{~cm}^{-3}$ [10]. For PLD plasmas, such densities are only present during the first few microseconds after target ablation. Nevertheless, this does provide an upper limit for the Stark broadening for the experimental settings used in this study. For instance, for the electronic transition of aluminium at $308.2 \mathrm{~nm}$, as used in chapter 4, the Stark broadening at a temperature of $5.000 \mathrm{~K}$ and an electron density of $10^{15} \mathrm{~cm}^{-3}$ is calculated to be $0.57 \mathrm{pm}$ [6]. As the Stark broadening reduces approximately linearly with the electron density, and the electron densities are expected to be significantly lower for our experimental settings as compared to this upper limit, it can be concluded that Stark broadening has no significant influence 
on the line broadening in our experimental observations.

Natural broadening When an ensemble of atoms in an excited state decays through spontaneous emission of radiation, the power of the radiation decreases exponentially vs. time. Therefore, unlike with oscillators that radiate with constant amplitude, the emitted radiation is no longer monochromatic, but shows a spectral broadening with a FWHM of [8]

$$
\delta v_{N}=A_{i} /(\pi)=1 /\left(\pi \tau_{i}\right)
$$

where $A_{i}$ is the spontaneous transition probability and $\tau_{i}=1 / A_{i}$ is the associated average lifetime of the excited state. Most of the lines corresponding to atomic transitions that are used in this study have lifetimes in the order of $10^{-8} \mathrm{~s}$, which corresponds to a natural broadening of the order of $10^{-2} \mathrm{pm}$. This means that the influence of natural broadening is negligible in PLD plasmas.

We conclude that of all broadening mechanisms discussed, Doppler broadening is dominant in the situations that we encounter in our experiments. Since Doppler broadening does not affect the spectrally integrated absorption cross-section as discussed in section 2.3, we can apply absorption spectroscopy to determine the calibration factor $C$ that links the signal from the camera detecting the LIF to the absolute density of the species in the plasma plume.

\subsection{Summary}

In section 2.1 we have discussed and suggested that laser-induced fluorescence is a very suitable technique to identify and map plasma constituents vs. time and space in PLD. In section 2.2 we have used the example of a two-level system to identify two regimes. The first is a linear regime at low laser intensity, where fluorescence is proportional to the excitation laser intensity and to the density of the excited species. The second is a saturated regime at high laser intensity, in which the fluorescence signal is no longer dependent on the laser intensity, but is still proportional to the species density. The linear regime is not suitable for quantitative measurements of particle density distributions, as any spatial inhomogeneities and shot-to-shot fluctuations in the laser beam would directly translate into inhomogeneities and fluctuations in the fluorescence intensity. However, these fluctuations are not present in the saturated regime, which is why we have chosen to perform all LIF measurements in this regime. Another advantage of performing LIF in the saturated regime is that the fluorescence signal is much stronger than in the linear regime, making measurements easier and providing a higher signal-tonoise ratio. To be able to relate relative density images provided by LIF to absolute densities of species in the plasma plume, a calibration is required. Of the several methods available, we have chosen absorption spectroscopy at low intensities. In section 2.3 we have discussed the basic principles behind AS and shown how to combine LIF and AS to create absolute density maps of plasma constituents. We 
have discussed the various line broadening mechanisms present in plasmas and concluded that, for our experimental parameters, Doppler broadening is the dominant mechanism. 


\section{References}

${ }^{1}$ A. Eckbreth, Laser diagnostics for combustion temperature and species, Vol. Volume 7, Energy and engineering science series, ISBN 0-85626-344-3 (Abacus Press, 1988).

${ }^{2}$ J. Daily, "Laser induced fluorescence spectroscopy in flames", Progress in Energy and Combustion Science 23, 133 (1997).

${ }^{3}$ S. Kawana, N. Sakurai, T. Fujii, M. Fukushima, N. Inoue, J. Matthews, S. Ogio, H. Sagawa, A. Taketa, M. Takita, S.B.Thomas, H. Tokuno, Y. Tsunesada, S. Udo, and L. Wiencke, "Calibration of photomultiplier tubes for the fluorescence detector of telescope array experiment using a rayleigh scattered laser beam", Nuclear Instruments and Methods in Physics Research A 681, 68 (2012).

${ }^{4}$ R. Al-Wazzan, C. Lewis, and T. Morrow, "A technique for mapping three-dimensional number densities of species in laser produced plumes", Review of Scientific Instruments 67, 85-88 (1996).

${ }^{5}$ C. Dutouquet and J. Hermann, "Laser-induced fluorescence probing during pulsed-laser ablation for three-dimensional number density mapping of plasma species", Journal of Physics D: Applied Physics 34, 3356-3363 (2001).

${ }^{6} \mathrm{H}$. Griem, Plasma spectroscopy (McGraw-Hill, 1964).

${ }^{7}$ W. Demtröder, Laser spectroscopy vol. 1: basic principles, 4th ed., Vol. 1 (Springer, 2008).

${ }^{8} \mathrm{~J}$. Broekaert, Analytical atomic spectrometry with flames and plasmas, ISBN: 3527-30146-1 (Wiley-VCH Verlag GmbH and Co. KGaA, 2002).

${ }^{9}$ D. Chrisey and G. Hubler, eds., Pulsed laser deposition of thin films (John Wiley \& Sons, Inc., 1994).

${ }^{10} \mathrm{C}$. Aragon and J. Aguilera, "Characterization of laser induced plasmas by optical emission spectroscopy: a review of experiments and methods", Spectrochimica Acta Part B: Atomic Spectroscopy 63, 893-916 (2008). 


\section{EXPERIMENTAL SETUP}

\section{Introduction}

The goal of the work carried out for this thesis is to provide a better understanding of the influence of external parameters in pulsed laser deposition, such as ablation laser fluence and background gas composition and pressure, on the plasma dynamics and chemistry.

To avoid perturbing the plasma dynamics and chemistry, we have chosen to analyze the plasma with optical spectroscopic techniques. Specifically, we have chosen laser-induced fluorescence (LIF) and absorption spectroscopy (AS) as tools that allow the detection and mapping of the plasma constituents. In the previous chapter we have discussed the theoretical aspects of these two techniques, and in this chapter we will give a description of the custom PLD setup in which we have implemented both LIF and AS. The challenges in setting up these experimental diagnostics in a fully functioning PLD chamber are discussed. Although LIF and AS are certainly proven spectroscopic techniques, applying them correctly is far from trivial and requires a specialistic design and technical expertise. The experimental setup had to be arranged such that it facilitates identification and localization of the constituents of a highly transient plasma with high selectivity and sensitivity, and high spatial and temporal resolution. Figure 3.1 shows a schematic of the full setup, with solid arrows depicting optical paths and dashed arrows representing electronic timing synchronization paths.

We will start in section 3.1 with a description of the central part of the setup, which is the PLD chamber where the plasma is being generated and analyzed. For the latter, the chamber was designed specifically to give access for optical diagnostics to the plasma. For the generation of the plasma, a target ablation system, including a $\mathrm{KrF}$ excimer laser was built, which will be described in section 3.2. The system generating the excitation radiation for LIF, indicated by the yellow blocks in figure 3.1, as well as the detection of LIF with an iCCD camera are discussed in section 3.3. Thereafter, in section 3.4, we describe the experimental confirmation 


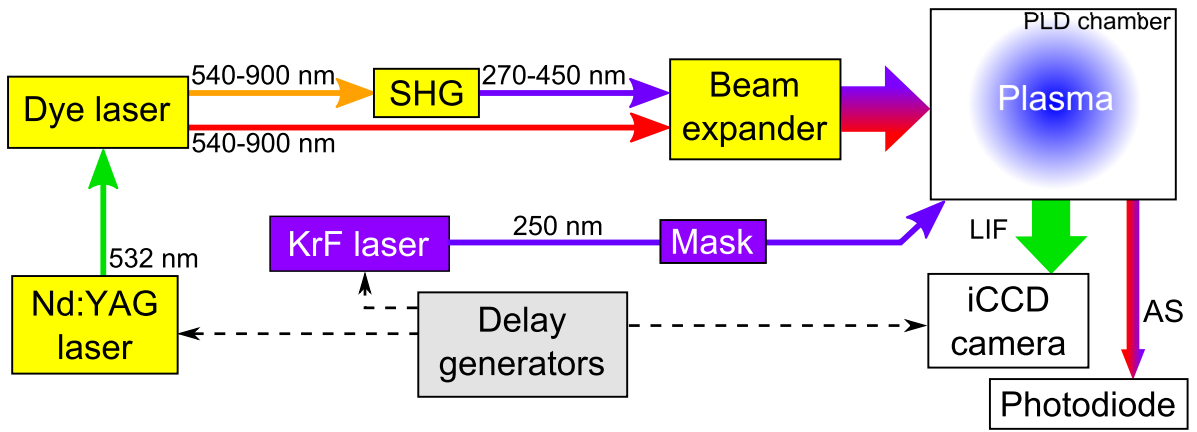

Figure 3.1: Schematic of the PLD setup including the system for the optical diagnostics of the ablation plasma. The ablation plasma is generated with a KrF laser (purple blocks). The excitation radiation for LIF is generated with a laser system consisting of a Nd:YAG and dye laser, with an option for frequency doubling to reach the UV by SHG (yellow blocks). The LIF is detected with an iCCD camera, and the absorption in the plasma is monitored with photodiodes. The timing synchronization is controlled with a set of digital delay generators.

of the linear and saturation regime, as was discussed in the theory (section 2.2). In section 3.5, we describe the experimental aspects of AS, including measurements to check the electronic response of the photodiodes as function of incident laser intensity. Finally, in section 3.6, we discuss the timing synchronization of all lasers and detectors using a set of digital delay generators.

\subsection{PLD chamber}

We have designed the PLD chamber specifically to allow for LIF and AS measurements on the plasma plume. For these purposes, the chamber is equipped with two pairs of two inline optical view ports that are mounted under an angle of \pm 45 degrees with the axis between target and substrate holder, as shown in figure 3.2. These angles were chosen instead of the more common 90 degrees angle in respect with the propagation direction of the plasma [1-3] to allow for measuring Doppler shifts [4] in the fluorescence of the plasma constituents from which velocity maps of the species can be generated. Unfortunately, we could not carry out any of these measurements within the time frame of this thesis.

On top of the chamber two more view ports are installed, one for letting the ablation laser enter, and one for imaging of LIF from the plasma onto an iCCD camera. All view port windows on the PLD chamber are made of fused silica which provides transmission over a broad wavelength range (from the UV to the IR), allowing access for both the excitation and ablation lasers as well as to the LIF from the plasma. The chamber does not contain a sample holder in order to obtain an unobstructed propagation of the excitation laser during plasma analysis. The dashed line in figure 3.2 indicates the position of a substrate, opposing the target, 
if films were to be grown.

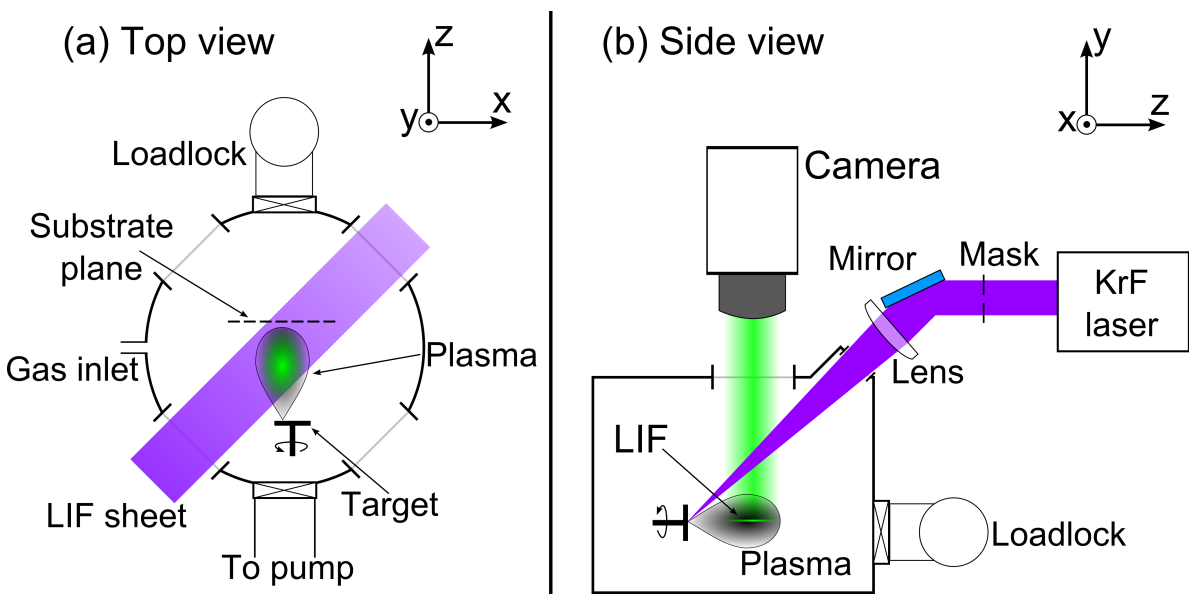

Figure 3.2: Schematic of the PLD chamber. (a) top view of the PLD chamber with two pairs of two inline ports allowing for a laser light sheet to pass through and excite the species in the plasma plume to generate LIF. The chamber does not contain a sample holder in order to obtain an unobstructed propagation of the excitation laser during plasma analysis. The dashed line indicates the position of a substrate, opposing the target, if films were to be grown. (b) shows a side view of the chamber with two ports on top. One port allows access to the excimer laser beam that is focused onto the target for ablation. The second port allows the iCCD camera to view the fluorescence from the plasma plume.

\section{Vacuum system, targets and substrate}

To ensure full control over the background atmosphere, the PLD chamber can be vacuum pumped to a base pressure of $2 \cdot 10^{-7}$ mbar. For this, the PLD chamber is equipped with a large turbomolecular pump (Pfeifer HiPace700, $685 \mathrm{~L} / \mathrm{s}$ ). A load lock is installed allowing target and substrate loading without having to pressurize the deposition chamber. This reduces a potential contamination of the chamber, and it speeds up the process of changing a target or substrate. The load lock is evacuated with its own pumping system (Pfeifer HiPace80, $9000 \mathrm{~L} / \mathrm{min}$ ) providing a pressure below $10^{-6}$ mbar within a few minutes.

To create a specific background atmosphere allowing us to study its influence on the propagation dynamics and chemistry in the plume, either pure or mixed gasses can be filled in the chamber ranging from $10^{-6}$ mbar up to $1 \mathrm{mbar}$ in pressure. The pressure in the chamber is controlled by creating a balance between inflow and outflow of the gas. For our experiments we use argon and oxygen which can be filled into the chamber using two gas flow controllers that can be set independently to a maximum inflow of up to $20 \mathrm{~cm}^{3} \mathrm{~s}^{-1}$ each. The target pressure is obtained by 
pumping gas out of the chamber through an adjustable butterfly valve between the PLD chamber and the vacuum pump that controls the outflow of gas.

A baratron gauge, for the range from 1.3 to $10^{-3} \mathrm{mbar}$, and a Pirani gauge (from $10^{-2}$ to $10^{-9}$ mbar) are used to measure the pressure inside the vacuum chamber over the entire 1 to $10^{-7}$ mbar range used during the experiments.

A target carousel with space for four targets can be loaded into the chamber through the load lock. By rotating the carousel, any of the four targets can be chosen for ablation. All targets are individually rotated as well, to prevent hole drilling by the ablation laser. The carousel allows targets with a diameter of maximum 1 inch and is adjustable to accommodate targets with a thickness of up to several centimeters.

Ablation of the targets is carried out using a $\mathrm{KrF}$ excimer laser, which will be discussed in the next section.

\subsection{Laser ablation system}

For target ablation, a high power excimer laser is most commonly used. Excimer lasers produce high energy pulses in the UV wavelength range, which is strongly absorbed by most PLD target materials, i.e., providing short absorption lengths. This is an important feature, because a short absorption length allows to generate a hot (several $10^{5} \mathrm{~K}$ ) and dense plasma leading to an efficient ablation. A long absorption length would only yield temperatures insufficient for ablation. For targets consisting of multiple chemical elements, heating would eject the materials through thermal evaporation of the target species. The stoichiometry of the plasma plume would in that case be dependent on the different vapor pressures of the target constituents [5]. Even with a stoichiometric target, differences in vapor pressure would lead to some target components evaporating at a higher rate than others, causing a non-stoichiometric transfer of materials towards the substrate, as well as changes to the composition of the target. It is therefore important to ensure target ablation instead of just target heating. Care must also be taken to ensure a uniform laser power over the entire illuminated area, also known as a top-hat distribution. This avoids that the target will only be heated in the lower intensity parts the beam, instead of ablated over the entire illuminated area.

For target ablation, we use a $\mathrm{KrF}$ excimer laser operating at a wavelength of $248 \mathrm{~nm}$ and a repetition rate of up to $10 \mathrm{~Hz}$ (Lambda Physik CompexPro 205). The laser provides up to $600 \mathrm{~mJ}$ of energy in a pulse of about $24 \mathrm{~ns}$ duration (FWHM).

A problem with excimer lasers is that they provide multi-mode beams with poor spatial coherence. In the near field, the beam has a rectangular shape determined by the discharge providing the optical gain for laser operation. Such beams cannot be losslessly converted into a top-hat distribution using optically transparent optical elements such as lenses and diffractive optics. However, an approximate top-hat intensity profile can be generated by illuminating a mask with the homogeneous center part of the laser beam and projecting the mask onto the target. In our setup, we use a mask with a hole of $15 \times 4 \mathrm{~mm}^{2}$. The rectangular shape is chosen 
as it matches the rectangular shape of the beam, thereby reducing the amount of the beam that is lost. Figure 3.3 shows a typical energy distribution behind the mask, as measured with a spatial beam profiler.

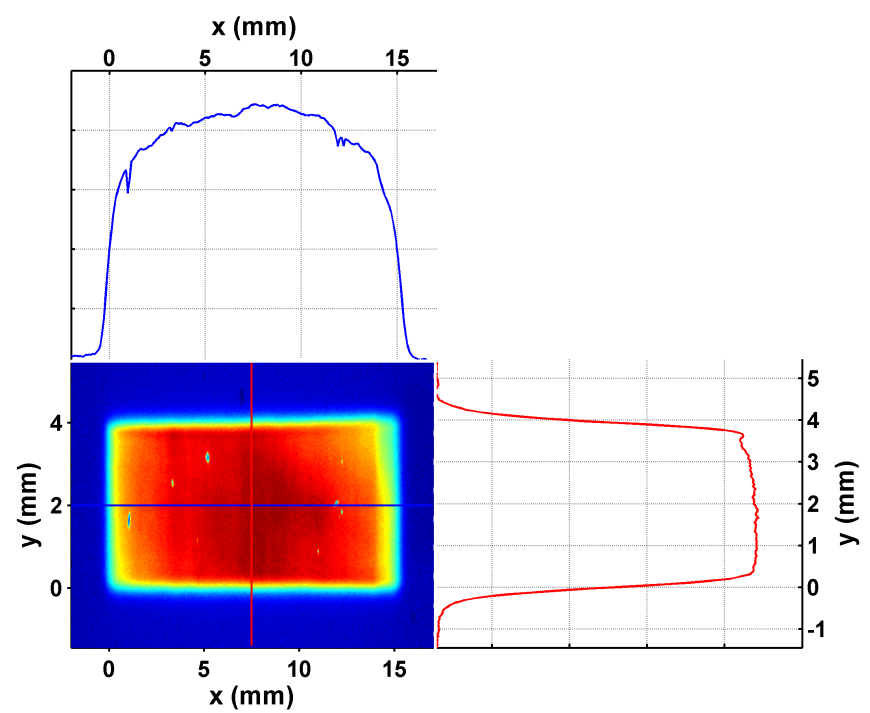

Figure 3.3: False color image of the spatial energy distribution in the KrF laser beam after passing through a rectangular hole of $15 \times 4 \mathrm{~mm}^{2}$ in the mask. The blue and red curves in the graphs show cross sections of the energy distribution along the blue and red lines, that is for $y=2 \mathrm{~mm}$, and $x=7.5 \mathrm{~mm}$, respectively.

To increase the fluence of the ablation beam to above the ablation threshold (typically $0.7 \mathrm{~J} / \mathrm{cm}^{2}$ for most perovskites), the beam has to be focused. To maintain the top-hat distribution of the beam on the target after passing through the mask, the mask itself is imaged onto the target using a lens placed close to the access port of the PLD chamber. The lens ( $\mathrm{f}=452 \mathrm{~mm}$ ) is placed at a distance of 51.7 $\mathrm{cm}$ from the target, and the mask is placed $358 \mathrm{~cm}$ from the lens. This creates a sharp image of the mask on the target with a demagnification factor of 6.9. As the target is illuminated by the ablation laser beam at an angle of incidence of $45^{\circ}$, the ablation spot possesses a size of $2.16 \times 0.82 \mathrm{~mm}^{2}$.

The plasma generated by the ablation laser is initially very hot and dense, leading to not only an ablation but also an excitation of internal degrees of freedom of the target constituents that may decay through spontaneous emission. Through a spectral analysis of the generated light, called optical emission spectroscopy (OES), various species in the plasma plume can be identified in the early stages of plume expansion. However, for later stages when the plasma has cooled down, spontaneous emission ceases and OES fails to work, which is actually the case for the most relevant stage of plume expansion, when the plume reaches the substrate location. In order to, nevertheless, extract spectral information in this stage of a dark plume, we have chosen for LIF, as will be discussed in the next section. 


\subsection{LIF system}

In this section we discuss the components required to generate and detect LIF. The technique requires a system that can generate tunable wavelength laser light with a sufficiently narrow spectral bandwidth, the latter to ensure that only a single transition of a single plasma species can be selected for excitation. Furthermore, the system needs to be tunable over a wide wavelength range to enable excitation of states in many different chemical elements and molecules. Most atomic species and small molecules have transitions that can be excited with light in the range of UV to near IR ( 250 to $900 \mathrm{~nm}$ ). A dye laser is most suitable for this task, because its output can be used either directly or can be first frequency doubled and then used for excitation. In terms of numbers, the output of the dye laser as used here can be tuned over a large range from $540 \mathrm{~nm}$ to $910 \mathrm{~nm}$. For excitation in the UV, the output of the dye laser can be frequency doubled using a second harmonic generation (SHG) setup, adding an extra wavelength range from $270 \mathrm{~nm}$ to 455 $\mathrm{nm}$. Our dye laser is pumped with a frequency doubled, Q-switched Nd:YAG laser.

\subsubsection{Laser system}

\section{Nd:YAG laser}

Optical pumping of the dye laser is provided by a frequency doubled Nd:YAG laser (Spectra-Physics Quanta-Ray DCR3). These lasers are commonly used for optical pumping, because they are reliable, and generate high-energy pulses and beams with high spatial and spectral coherence, in combination with a high peak power, which allows an efficient conversion of its output. The Nd:YAG laser used here operates at a wavelength of $1064 \mathrm{~nm}$ and generates $1 \mathrm{~J}$ output energy per pulse at a repetition rate of $10 \mathrm{~Hz}$. The optical output is frequency doubled to a wavelength of $532 \mathrm{~nm}$ via a $\mathrm{KD} * \mathrm{P}$ crystal that is integrated in the laser system. To improve the temporal profile of the output pulse, the Nd:YAG laser is injection seeded with a diode pumped single-frequency continuous-wave Nd:YAG laser (MISER ${ }^{1}$ ). Seeding has the additional advantage that it significantly reduces the timing jitter of the laser output pulse with respect to the pulse triggering the laser, to a value of below 1 ns. Without seeding, the temporal jitter of the laser output would amount to tens of nanoseconds. The strong reduction of the jitter allows for an accurate synchronization of the Nd:YAG laser with the other systems in the PLD setup.

The temporal profile of the Nd:YAG laser's second harmonic ouput (532 nm), along with that of the output of the dye laser and its second harmonic (SH), is shown in figure 3.4. The pulse length (FWHM) of the Nd:YAG laser at $532 \mathrm{~nm}$ is $8.4 \mathrm{~ns}$, of the dye laser shows a pulse duration of $5.4 \mathrm{~ns}$ and of its $\mathrm{SH}$ a pulse duration of $3.0 \mathrm{~ns}$.

\footnotetext{
${ }^{1}$ Monolithic, Isolated, Single mode, End pumped, Ring laser
} 


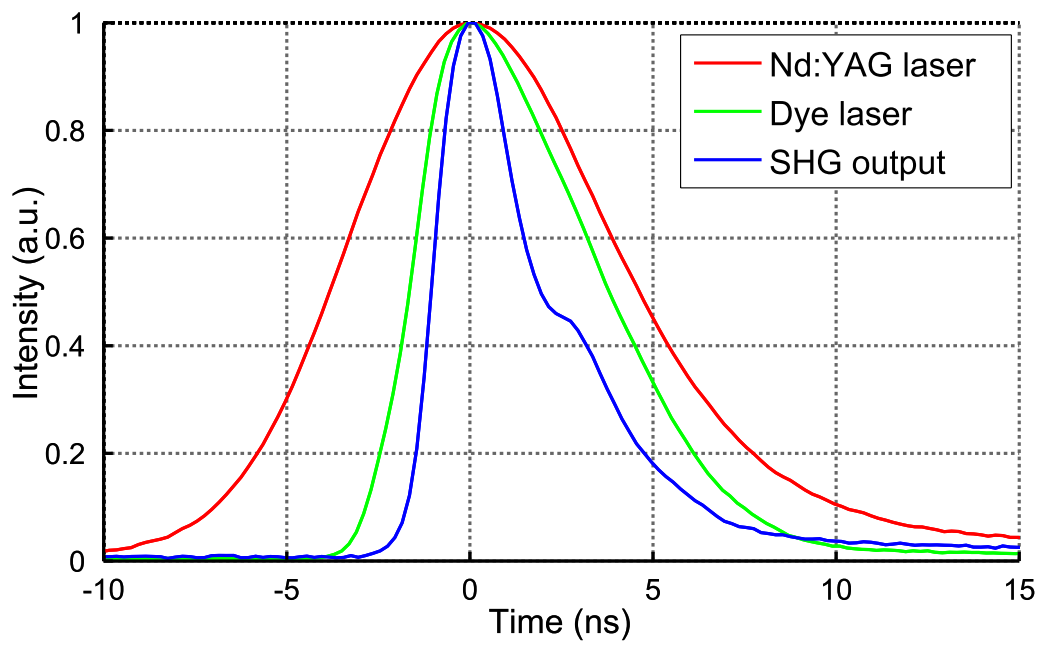

Figure 3.4: Comparison of the typical temporal profiles of the Nd:YAG laser's second harmonic output (red trace, $532 \mathrm{~nm}, 8.4 \mathrm{~ns}$ FWHM), of the dye laser (green trace, $5.4 \mathrm{~ns}$ FWHM) and of the frequency doubled output of the dye laser (blue trace, 3.0 ns FWHM) used in the experiments. All intensities are normalized to unity.

\section{Dye laser}

The dye laser (Spectra-Physics Quanta-Ray PDL-2) can be operated with various different organic dyes, typically dissolved in methanol, each generating light in a specific wavelength range, as shown in figure 3.5. Various different dyes were chosen during the course of the experiments to match the excitation wavelengths required for LIF from the various selected species of interest. The dye laser comprises an oscillator and an amplifier. The oscillator allows to select a specific wavelength within the wavelength range of the dye. The amplifier boosts the oscillator output to a level of tens of mJ's required to provide sufficient intensity for performing LIF in the saturation regime, as was discussed in section 2.2.

The spectral bandwidth of the dye laser output is measured using a scanning confocal Fabry-Perot interferometer (FPI) with a free spectral range (FSR) of 10 $\mathrm{GHz}$ and a resolution of $67 \mathrm{MHz}$, which corresponds to a finesse of 150 .

The blue symbols in figure 3.6 show the photodiode signal measured at the output of the interferometer during a scan of the cavity length of the FPI while the wavelength of the dye laser was fixed at $614.00 \mathrm{~nm}$. The red curve is the calculated transmission function of the given FPI if it were recorded with strictly monochromatic light. In our experiments, however, where the input light to the FPI is not strictly monochromatic, the output from the FPI consists of a convolution of the spectral shape of the input light with the transfer function of the FPI. As the resolution of our FPI is significantly smaller than the features in the measured transmission spectrum, the transfer function can be approximated with a 


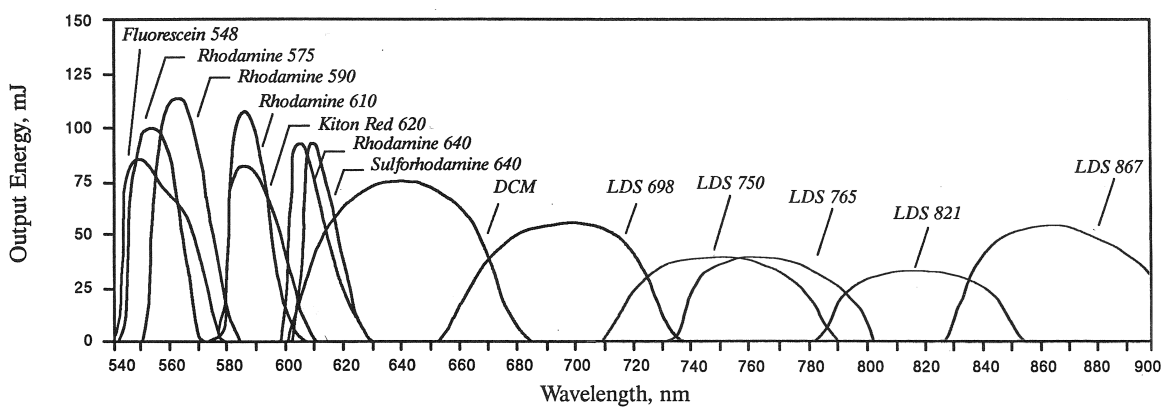

Figure 3.5: Typical operating spectral ranges and corresponding maximum output energy per pulse for dyes optically pumped with laser pulses of $1 \mathrm{~J}$ at a wavelength of $532 \mathrm{~nm}[6]$.

delta function, while the laser bandwidth is in the order of the width of the transmission peaks. More specifically, the measured data points do not drop to zero in between the peaks, which indicates that the spectral width of the input beam is comparable to the FSR of the FPI $(10 \mathrm{GHz})$. As the measured signal is repeated every $10 \mathrm{GHz}$, an input broader than $10 \mathrm{GHz}$ would therefore partially overlap. To account for this, we have fitted curves of several different shapes only to the data between -4 and $4 \mathrm{GHz}$. We found that a Gaussian curve provided the most accurate fit to this data. This fit is plotted in figure 3.6 as the green curve. Finally, to verify that the chosen shape and width also fit the entire recorded spectrum, we have calculated the convolution of the Gaussian line profile with the FPI transfer function, plotted as the black curve. It can be seen that the convolution corresponds well with the measured data. From the Gaussian fit a linewidth of $5.7 \mathrm{GHz}$ (FWHM) is determined, corresponding with $7.2 \mathrm{pm}$ (FWHM). As we have discussed in section 2.4 , the expected linewidth of the atomic species is around 2 or $3 \mathrm{pm}$. This means the dye laser has a full overlap with the absorption bandwidth, ensuring that all particles will be excited.

The temporal profile of the dye laser output is shown in figure 3.4 as the green trace. The asymmetric shape is caused by a fixed time delay between the oscillator and the amplifier of the dye laser. This delay is set to ensure that the spontaneous emission from the oscillator, which is present in the first nanoseconds when lasing is still building up, is not amplified. This spontaneous emission is much more broadband than the lasing output, and should therefore be avoided.

\subsubsection{Second harmonic generation}

Most atomic species have transitions and corresponding emission wavelengths not only in the visible and NIR wavelength range (450 to $900 \mathrm{~nm}$ ), but also in the UV $(<450 \mathrm{~nm})$. For LIF measurements, it is often preferable to use transitions in the UV instead of the visible wavelength range, because these have a higher transition probability leading to stronger LIF signals, which are easier to detect. For analyzing 


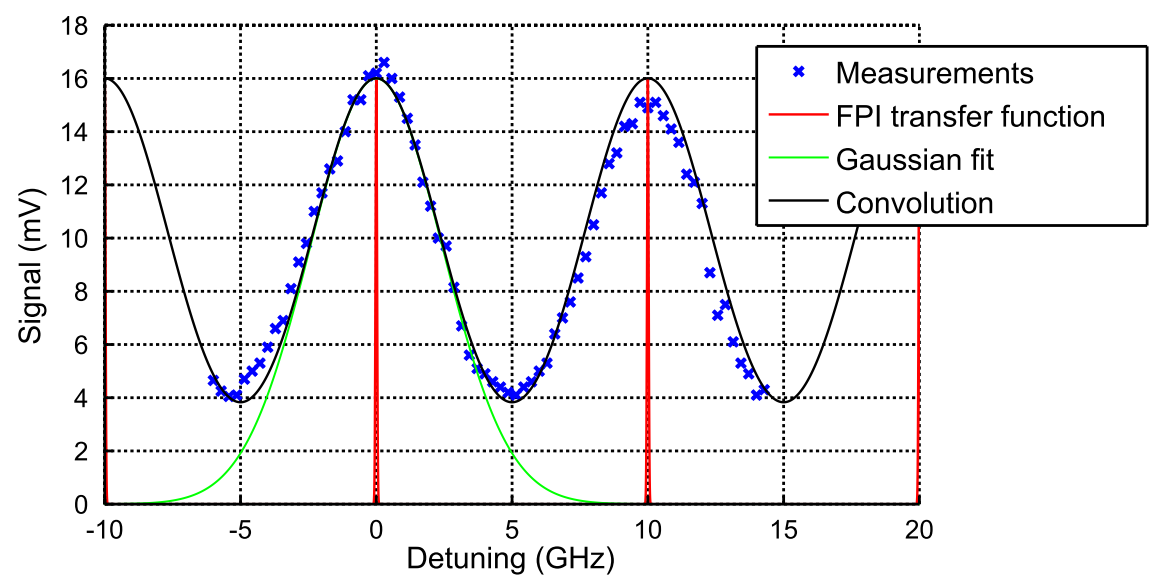

Figure 3.6: Measurement of the output signal of the photo diode attached to a scanning Fabry-Perot interferometer with a FSR of $10 \mathrm{GHz}$ and a finesse of 150. The input is the laser beam of the dye laser tuned to $614.00 \mathrm{~nm}$. Scanning is done by changing the cavity length. The blue symbols show the measured data. The red curve shows the FPI transfer function. A Gaussian fit to the measured data between -4 and $4 \mathrm{GHz}$ is plotted as the green curve. In black is plotted a convolution of the Gaussian fit with the FPI transfer function.

excitation in the UV we built a second harmonic generation setup that can convert the visible output of the dye laser into the UV range, as shown in figure 3.7.

Figure 3.7 shows a schematic top view of our SHG setup. The output beam from the dye laser enters on the top right (yellow line) and is reflected towards the SHG crystal (BBO) via three mirrors. Two pinholes are placed in the beam path for alignment as well as spatial filtering, transforming the dye laser output into a circular beam. A Galilean telescope demagnifies the laser beam cross section by a factor of 3.33, which increases the intensity by a factor of about 11 . This is done to increase the efficiency of the SHG process, as it scales quadratically with the input intensity, which in this case is increased approximately by a factor of 120 . Special care is taken to maintain a parallel beam in the crystal. The dye laser beam then passes through a BBO crystal in which the SHG takes place. With a Pellin Broca prism, the dye laser beam and its $\mathrm{SH}$ are spatially separated, after which the dye laser beam is sent into a beam dump, and the SH is directed to the PLD chamber.

The BBO crystal used in our setup $\left(8 \times 6 \times 10 \mathrm{~mm}^{3}\right.$ in size $)$ is cut at $\theta=36^{\circ}$ and $\phi=90^{\circ}$. These angles were chosen to allow for angle-tunable phase matching of second harmonic generation at a fundamental wavelength range of 540 to $900 \mathrm{~nm}$. Anti-reflection (AR) coatings are applied on both input and output facets, with the front coated for 540 to $900 \mathrm{~nm}$ and the back for 270 to $450 \mathrm{~nm}$. The average acceptance angle over the tuning range of the BBO crystal is $0.51 \mathrm{mrad}\left(0.029^{\circ}\right)$ and the average acceptance bandwidth is $10.43 \mathrm{~cm}^{-1}(0.454 \mathrm{~nm})$ [7]. The average 


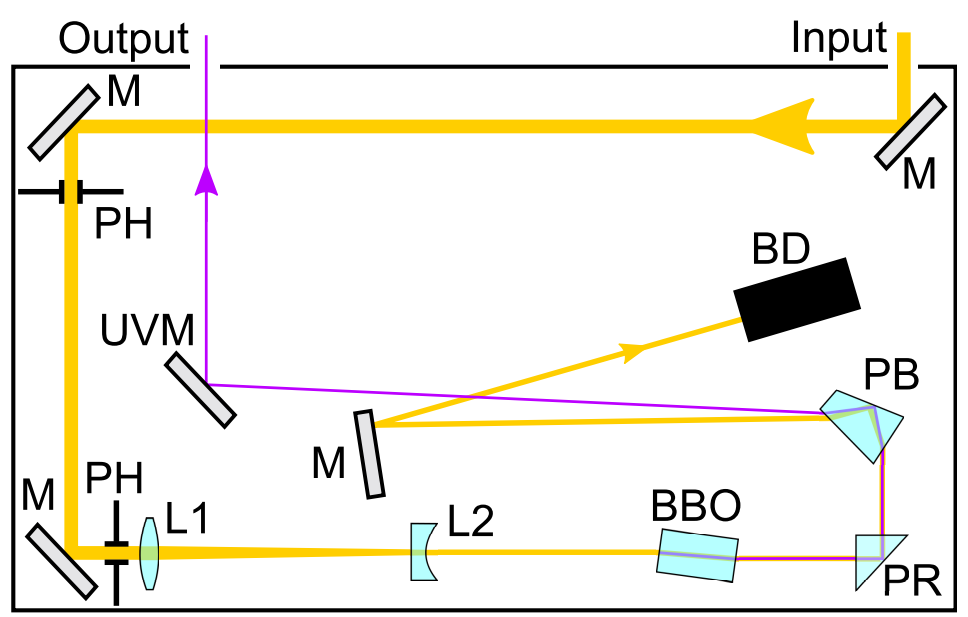

Figure 3.7: Setup for frequency doubling of the dye laser output used for excitation in the UV wavelength range, from $270 \mathrm{~nm}$ to $450 \mathrm{~nm}$. The input from the dye laser (yellow) is on the top right. (UV)M $(U V$-)mirror, $P H=$ pinhole, L1: $f=250 \mathrm{~mm}$, L2: $f=-75 \mathrm{~mm}, P R=$ prism, $P B=$ Pellin Broca prism, $B D=$ beam dump.

conversion efficiency of this SHG setup is approximately $10 \%$. This is lower than the maximum value of $40 \%$ that is typically possible. However, for our setup, an efficiency of $10 \%$ is well sufficient to generate pulses that can drive the LIF into saturation, as will be discussed in section 3.4. Also, one of the most effective ways of increasing the output efficiency is to increase the input energy density of the BBO crystal, either by increasing the input pulse energy or by a stronger focus of the input beam. However, this would bring the input energy closer to the damage threshold of the BBO crystal, which is to be avoided. The fluctuation in the shot-toshot output energy is approximately $10 \%$, but as the LIF measurements are carried out in the saturation regime, the LIF power remained largely independent of these fluctuations.

\subsubsection{Sheet formation and characterization}

To probe a cross section of the plasma plume, the circular output beam from the dye laser or SHG module has to be transformed into a thin, wide planar light sheet providing the excitation for LIF. To achieve this, two Galilean telescopes are placed in series, consisting of both cylindrical and spherical lenses, to expand the beam in the $y$-direction and focus it in the $\mathrm{x}$-y plane, as shown in figure 3.8. Special care is taken to prevent light from hitting the edges of the lenses, to avoid light scattering, which is achieved by using diaphragms.

The first telescope has the purpose to expand the beam four-times in the $\mathrm{x}-\mathrm{z}$ plane in the coordinate system as defined in figure 3.2. The telescope comprises two cylindrical lenses (CL1: $\mathrm{f}=-25 \mathrm{~mm}, \mathrm{CL2}: \mathrm{f}=100 \mathrm{~mm}$ ). The second telescope 


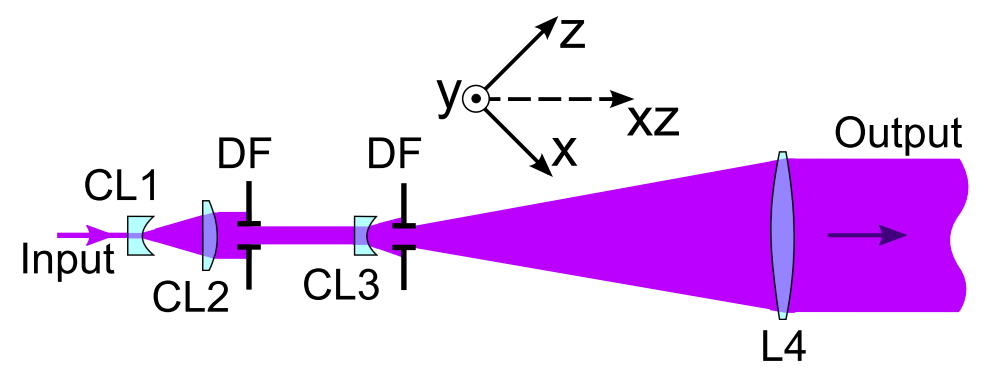

Figure 3.8: Two Galilean telescopes in series to expand the LIF excitation beam into a thin light sheet. DF: diaphragm, (C)L: (cylindrical) lens. CL1: $f=-25 \mathrm{~mm}, C L 2$ : $f=100 \mathrm{~mm}, C L 3: f=-25 \mathrm{~mm}, L 4: f=500 \mathrm{~mm}$. Coordinate system as defined in figure 3.2

serves to expand the beam further in the x-z plane by another 20 times into a parallel planar light sheet, and simultaneously focusing it in the y-direction to a thickness of approximately $350 \mu \mathrm{m}$, with the focus centered at the line between the ablation target and the deposition substrate. This second telescope consists of a cylindrical and a spherical lens (CL3: $\mathrm{f}=-25 \mathrm{~mm}, \mathrm{~L} 4: \mathrm{f}=500 \mathrm{~mm}$ ).

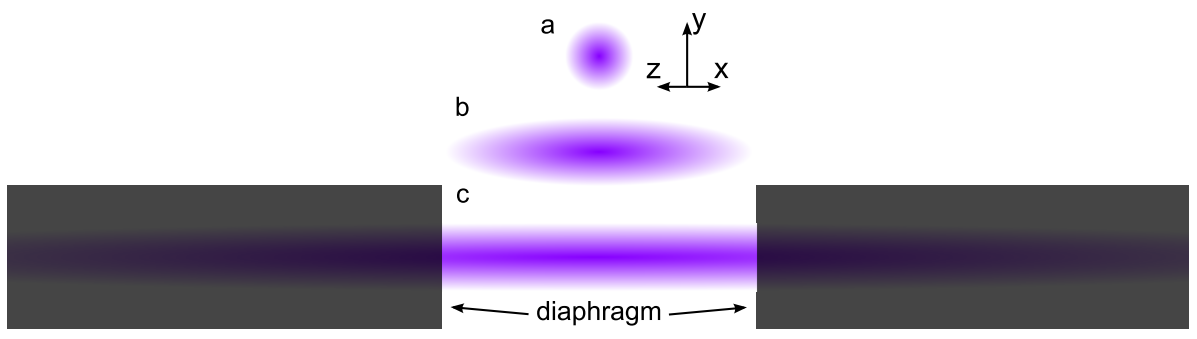

Figure 3.9: Schematic representation of the spatial profile of the LIF excitation laser beam. (a) Circular Gaussian beam as produced by the dye laser or SHG setup. (b) Elliptical beam profile after a four-times expansion in the $x-z$ plane. (c) After expanding the beam 80 times in the $x-z$ plane and selecting only a small section from the middle using a diaphragm, a beam with near-homogeneous intensity profile in the $x$-z plane is obtained. Coordinate system as defined in figure 3.2

The large expansion factor of 80 in the $\mathrm{x}-\mathrm{z}$ plane with an aspect ratio of about 500 is chosen to generate a planar sheet with a near-homogeneous intensity profile in the z-y plane. As the input for the planar sheet is close to a Gaussian beam that shows a gradual, circularly symmetric intensity variation (figure 3.9a), simply expanding the beam with a factor to make it wide enough for our purpose (approximately $5 \mathrm{~cm}$ ) would result in an elliptical spatial beam profile, as shown in figure 3.9b. Even when focusing the beam in the y-direction, a significantly higher intensity would be present in the center of the planar sheet compared to its edges in the $\mathrm{x}$ and $\mathrm{y}$-directions. To avoid this, we expanded the beam to a size significantly 
larger than $5 \mathrm{~cm}$ (about $25 \mathrm{~cm}$ ) and cut out the center part, as shown in figure 3.9c. The Gaussian intensity profile along the y-axis is still present and its effect on the measurements is discussed in section 3.4.

Figure 3.10 shows a measurement of the thickness of the beam (FWHM) around the focus along the propagation direction (the xz-direction, as defined in figure 3.8) for a typical light sheet. The red curve is a hyperbolic fit to the data, and the overall agreement confirms propagation as in a Gaussian beam focus. As can also be seen, the illuminated volume changes depending on the xz-position in the plasma plume. As LIF measurements are carried out in the saturation regime, the LIF signal is proportional to both the species density and the illuminated volume (i.e. proportional to the beam radius in figure 3.10). To retrieve only the density we corrected the recorded fluorescence maps for the spatial dependence of the illuminated volume. This is done separately for each of the used excitation wavelengths.

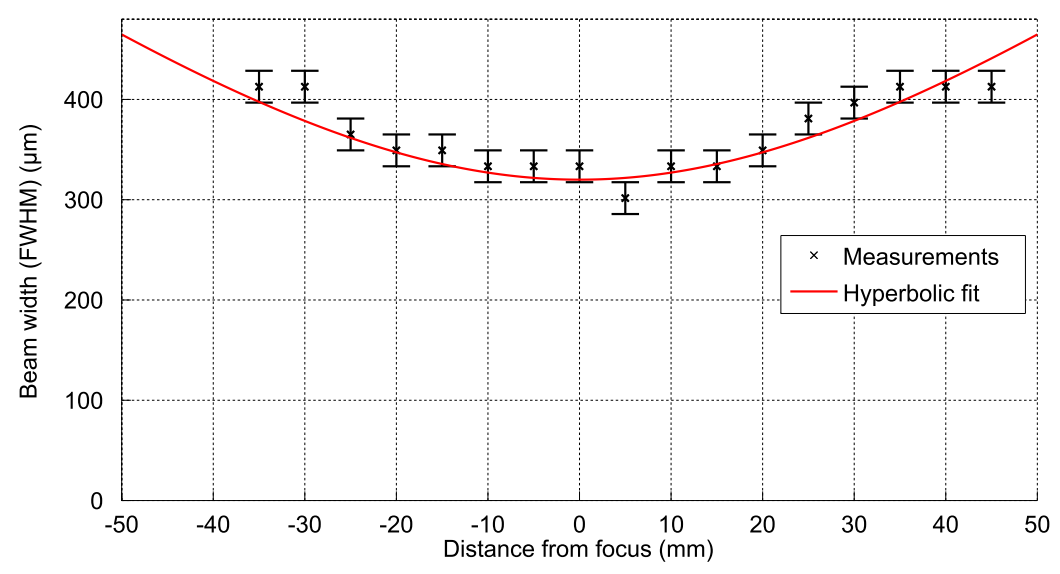

Figure 3.10: Measured thickness of the light sheet (FWHM) along the xz-direction as function of the distance from the focus (black symbols) and a hyperbolic fit to the data (red curve). The specific measurement of the sheet thickness shown here was performed for determining the Ti density in the plasma plume described in chapter 5 , for an excitation wavelength of $294.2 \mathrm{~nm}$.

\subsubsection{Detection}

The planar laser sheet illuminates a cross section of the plasma plume, generating LIF. To capture the spatial distribution of the LIF, a camera is placed perpendicular to the planar laser sheet, as shown in figure 3.2. We choose an intensified CCD camera (iCCD camera) which provides a high sensitivity for wavelengths in the range from UV to NIR. This allows to detect low densities or weakly fluorescing species, as well as a large range of different species.

Furthermore, we chose a camera with an ultrashort shutter time of down to 
0.5 ns that allows to capture only the pulsed emission of LIF immediately after the excitation light is injected. Thereby the capture of spontaneous emission from the plasma as caused by the ablation laser can be strongly suppressed, as such spontaneous emission might still be present at shorter delay times from target ablation (around $10 \mu s$ ).

\section{iCCD camera}

The laser-induced fluorescence is detected using an iCCD camera (Standford Computer Optics 4Picos) which is positioned perpendicular to the planar laser excitation sheet, as shown in figure 3.2. The LIF in the plasma plume is imaged onto the photocathode of the camera, using a homemade UV zoom lens system. The photocathode is followed by a Micro Channel Plate (MCP) intensifier with phosphor screen. The intensified image on the phosphor screen is captured by a CD chip. The spectral sensitivity of the photocathode ranges from $165 \mathrm{~nm}$ to $820 \mathrm{~nm}$. The MCP intensifier, besides significantly amplifying the input signal, also functions a very fast shutter (down to $0.5 \mathrm{~ns}$ ), as it can be rapidly switched on and off.

\section{Camera amplification factor}

Due to expansion of the plume and chemical reactions, the density of many plasma constituents, especially atomic species, will decrease at longer delay times with respect to target ablation. This will lead to a decrease of the LIF signal of orders of magnitude. To still be able to detect these small LIF signals, the amplification of the iCCD camera can be increased accordingly. However, then a direct comparison of LIF signals from a specific species measured at different amplification settings can only be carried out if the relative amplification factor for these settings is determined by measurement. To this end, we have measured the relative amplification factor of the image intensifier as function of the MCP voltage, for which we have arbitrarily chosen a value of $600 \mathrm{~V}$ to correspond to an amplification factor of 1 . A Helium-Neon laser beam is expanded to cover most of the photocathode. Calibrated grey filters are then used for controlled attenuation of the intensity of the beam. This allows to increase the amplification of the iCCD camera without driving it into saturation. By comparing the optical power directed into the camera with the corresponding signal and MCP voltage, a relative amplification factor as function of MCP voltage can be determined.

In figure 3.11, the relative amplification factor as function of the applied voltage on the MCP is plotted. The data points in the graph are normalized to unity in respect to the measurement at an MPC voltage of $600 \mathrm{~V}$ and fitted with a spline curve that is used in all measurements to account for the use of different MPC voltages at different delay times from target ablation. With the exception for very short shutter opening times ( $<5 \mathrm{~ns}$ ), we found no dependence of the amplification factor on the shutter opening time. It should be noted that this calibration allows for comparing the LIF signal at different locations and times of a plasma plume, but it does not allow to directly compare LIF measurements of different materials. The reason for this is that several factors are not taken into account in this calibration, 
such as the spectral variation of the response of the photocathode, as well as the wavelength dependent transmission of the lens system and spectral filters.

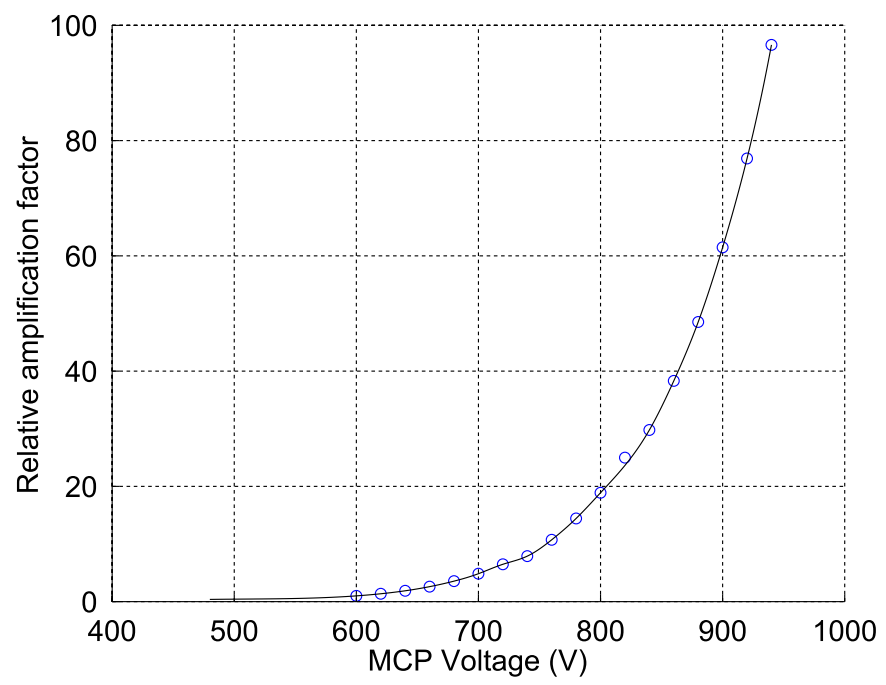

Figure 3.11: Relative amplification factor of iCCD camera versus MCP voltages (blue circles), normalized to unity at an MPC voltage of $600 \mathrm{~V}$. The spline fit curve (black line) is used as relative calibration for measurements of LIF done at different MCP voltages.

\section{Imaging system with zoom lens and iCCD camera}

A custom built zoom lens system consisting of a pair of quartz plano-convex lenses (two-inch diameter, $\mathrm{f}=150 \mathrm{~mm}$ ) is used to image the LIF onto the iCCD camera. The field of view of the imaging system, including the zoom lens and the iCCD camera, is matched to the distance between the target and substrate to optimize the spatial resolution of the imaging system. Using a calibrated resolution target (Thorlabs Negative NBS 1963A Resolution Target, 2" $\times 2$ ", 1 to 18 cycles $/ \mathrm{mm}$ ), the spatial resolution of this imaging system is determined at approximately $300 \mu \mathrm{m}$. For a length reference in the LIF images, the distance between target and substrate is used which is well defined and amounts to $50 \mathrm{~mm}$. Focusing is done by placing a thin, two-mm post in the middle of the planar LIF excitation sheet, perpendicular to the sheet. This creates a sharp shadow in the spatial LIF image, which is used to find the optimum focusing conditions.

\section{LIF detection}

The detectability of the LIF signal is often reduced, or sometimes completely overwhelmed, by broadband spontaneous emission and blackbody radiation of the 
plasma due to thermal excitation in the plume. Even though the spontaneous emission is significantly reduced as the plasma expands and cools down, the volume of the plasma that is excited with LIF is much smaller than the total volume of the plasma plume $(<1 \%)$. This means that even very weak spontaneous emission can generate a signal on the camera comparable to the LIF signal. To reduce this effect, we use spectral bandpass filters and apply a background subtraction to all measurements. Bandpass filters, either colored glass or dielectric interface filters, can be inserted into the lens system, allowing only the LIF signal and a small portion of the thermal emission of the plasma to pass. This strongly increases the signal-to-noise ratio (SNR) of the measurements. To remove the residual background of thermal emission that is still transmitted by the spectral filter, a measurement of the thermal emission (with the LIF laser turned off) is subtracted from the LIF measurement (with the LIF laser turned on).

In few cases, the scattering of the laser excitation beam on the windows and interior of the PLD chamber generates a significant amount of background on the camera. To remove such scattering background, measurements with only the LIF beam and no ablation plasma are subtracted from the LIF measurements after the thermal emission background has been subtracted.

To minimize the influence of the shot-to-shot variation in laser pulse energy of both the ablation laser and LIF beam, all measurements are averaged over 30 shots. Averaging over more than 30 shots is found to improve the measurements no further. Averaging also reduces small fluctuations in intensity in the x-z plane of the LIF sheet, which are caused by small shot-to-shot variations of the beam shape of the dye laser output.

The lowest particle density this system was capable of detecting in a plasma is $2 \cdot 10^{9} \mathrm{~cm}^{-3}$ (Ti in an STO plasma). However, this is not an absolute limit, as this number depends on many different factors, such as the emission rate of the species at the fluorescence wavelength and the temperature and composition of the plasma. The limiting factor in the current setup is the background noise produced by the MCP intensifier, which increases with increasing amplification.

\subsection{LIF measurements in saturation regime}

Previously, we showed in section 2.2 that in the saturation regime the LIF intensity becomes independent of the intensity of the excitation laser and scales proportional with the density of the excited species (see equation 2.16). Because this makes the LIF measurements largely immune to spatial inhomogeneities and shot-to-shot fluctuations in the excitation laser beam, we want to carry out our measurements in this regime. To ensure that we measure LIF in the saturation regime, it is important to investigate at what laser excitation intensity the LIF intensity starts to saturate. The saturation intensity, $I_{\text {sat }}^{v}$, is defined in section 2.2 as

$$
I_{s a t}^{v} \equiv \frac{\left(A_{k i}+Q_{k i}\right) c}{B_{i k}+B_{k i}}
$$


which depends on the collisional quenching rate, $Q_{k i}$, and the Einstein coefficients, $A_{k i}, B_{i k}$ and $B_{k i}$, of the electronic transition that is being excited. Because of the low density in the plasma plume and the background $\left(<10^{15} \mathrm{~cm}^{-3}\right)$ we expect that the quenching rate for the excited state is in the order of $\mu \mathrm{s}$. This is about two to three orders of magnitude smaller than the inverse lifetime $A_{k i}$ of the excited level and therefore negligible. Furthermore, the optical transitions we have chosen for the elements investigated in this thesis (Al, Ti, Y and Bi) have very similar Einstein coefficients, which means that their respective saturation intensities will have very similar values. Because the LIF measurements are carried out at excitation laser intensities several times larger than $I_{\text {sat }}^{v}$, as will be shown below, we have only done a detailed saturation measurement for a single element, Ti. For the other elements, only a visual check was performed, in which it was verified that the LIF intensity did not increase visibly with an increasing excitation laser intensity.

To illustrate our approach to determine the saturation intensity, we have measured the LIF intensity from $\mathrm{Ti}$ in an plasma generated with a $\mathrm{SrTiO}_{3}$ target (STO) as function of the excitation pulse energy. The excitation pulse energy is varied by inserting different combinations of grey filters with calibrated optical density (OD filters).

For excitation we make use of the $294.2 \mathrm{~nm}$ transition in neutral $\mathrm{Ti}$, which is a transition from the atomic ground state $\left(3 d^{2} 4 s^{2} a^{3} F\right)$ to the $3 d^{2}\left({ }^{1} G\right) 4 s 4 p\left({ }^{3} P^{\circ}\right) v^{3} F^{\circ}$ state, to study the relation between excitation laser intensity and the LIF intensity. At this wavelength, the maximum pulse energy that can be generated in the excitation laser sheet is $80 \mu \mathrm{J}$.

The LIF signal, resulting from relaxation to the $3 d^{3}\left({ }^{4} F\right)^{4} s b^{3} F$ state at several wavelengths around $445 \mathrm{~m}$, is averaged spatially over a $5 \times 5 \mathrm{~mm}^{2}$ area in the center of the plasma plume, as well as over 30 shots for each setting of the pulse energy of the excitation laser.

The results of the saturation measurement are plotted in figure 3.12, showing the relative LIF signal, with the maximum value normalized to unity, as function of the excitation laser energy (blue crosses). The insert with data recorded at low pulse energies shows a linear relation between the LIF and the excitation pulse energy, which can been seen from a linear fit to the six lowest-energy data points (below $0.5 \mu \mathrm{J}$ ). However, from the fit it can also clearly be seen that the LIF signal ceases to scale linearly with the excitation energy for pulses with an energy above $0.5 \mu \mathrm{J}$. The graph shows that the LIF signal saturates at high excitation energies, similar to the predicted response plotted in figure 2.3, though there is a deviation at higher LIF excitation energies. Here, we observe that the LIF signal does not converge to a fixed value as predicted in figure 2.3. Instead, a small positive slope of the LIF signal with the excitation energy appears to remain. We assume that the reason for this deviation from the predicted behavior is that in our experiments, we use a laser beam that possesses in the y-direction a Gaussian intensity distribution (see figure 3.9) instead of a uniform intensity distribution (a so-called "top-hat" beam) that was intrinsically assumed to model the saturation in section 2.2. The effect of a non-uniform intensity is that the degree of saturation of the LIF becomes dependent on the location in the excitation laser beam (here the y-coordinate), 


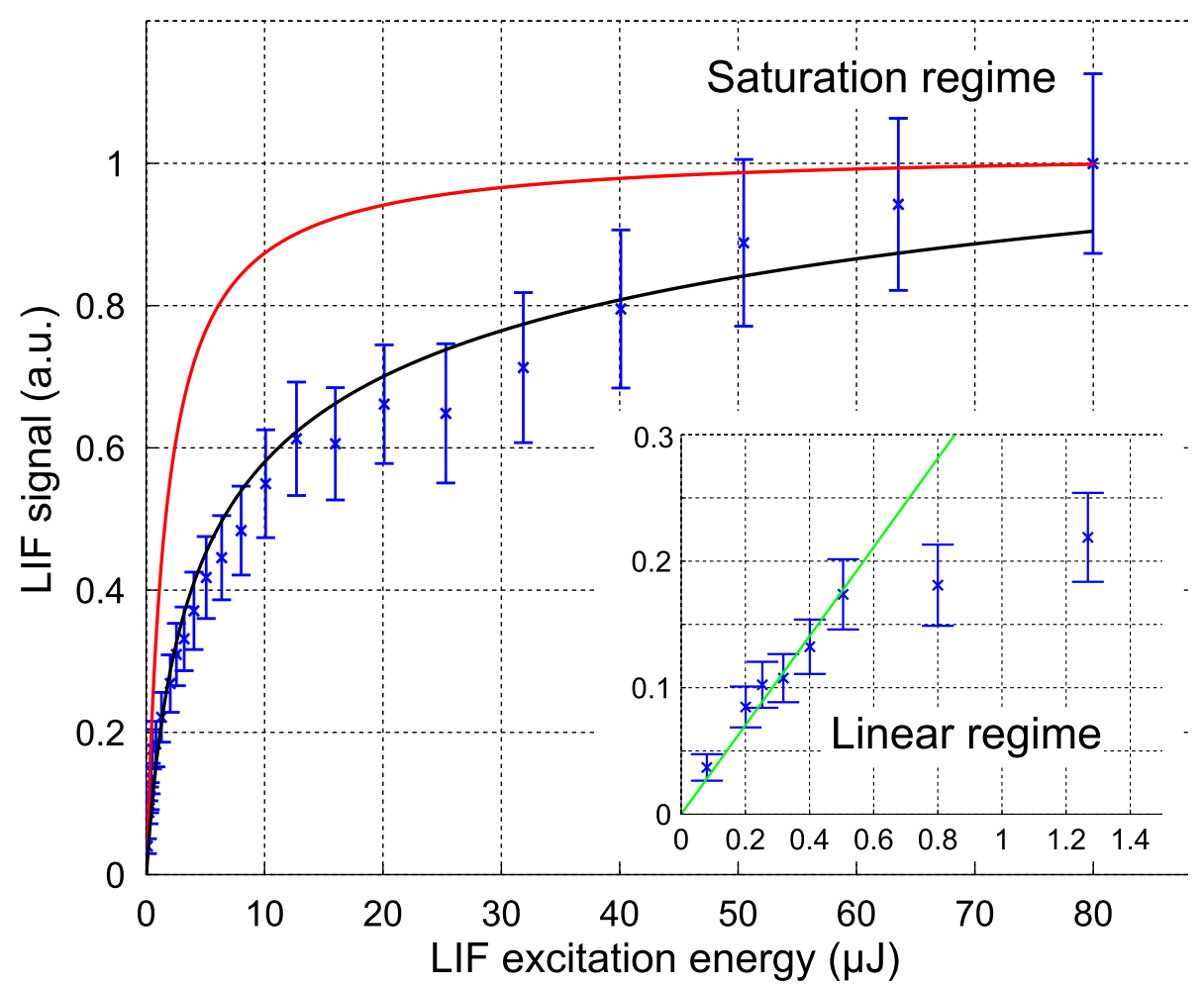

Figure 3.12: LIF signal (blue crosses) from neutral Ti measured with an iCCD camera as a function of the energy of the excitation laser in the planar lightsheet. The neutral $\mathrm{Ti}$ is excited at a wavelength of $294.2 \mathrm{~nm}\left(3 d^{2} 4 s^{2} a^{3} F \rightarrow\right.$ $\left.3 d^{2}\left({ }^{1} G\right) 4 s 4 p\left({ }^{3} P^{\circ}\right) v^{3} F^{\circ}\right)$ and fluoresces at several wavelengths around $445 \mathrm{~nm}$ (relaxation to the $3 d^{3}\left({ }^{4} F\right) 4 s b^{3} F$ state). The measurements are carried out at $30 \mu \mathrm{s}$ after the ablation of the target, in a background of 0.1 mbar Ar. Each data point is an average of 30 measurements. The black curve is calculated for a laser light sheet with a Gaussian beam profile, the red curve is calculated for a laser light sheet with a uniform beam profile (top hat). The inset shows an enlarged view of the LIF signal for low excitation energies ranging from 0 to $1.4 \mu \mathrm{J}$. The green line is a linear fit to the data points. 
depending on the function which describes the excitation beam profile, $I(y)$.

To illustrate the influence of a Gaussian beam profile on the spatial variation of saturation of the LIF signal, we have rewritten equation 2.14 with a spatially depending excitation laser to

$$
F(y)=C_{k i} \frac{I(y)}{1+\frac{I_{s a t}}{I(y)}}
$$

where $F(i)$ is the fluorescence intensity at position $y$ in the laser excitation beam, $C_{k i}$ summarizes all other physical parameters, such as the Einstein coefficients for this transition, $I(y)$ is the laser excitation intensity at position $y$ and $I_{\text {sat }}$ is the saturation intensity. In this equation we inserted for $I(y)$ both a Gaussian and a top-hat intensity distribution across the laser excitation light sheet assuming a homogeneous thickness in a homogeneous medium. The spatially integrated LIF signal calculated for a Gaussian distribution has been fitted to the experimental data and plotted as the black curve in figure 3.12. The variables $C_{k i}$ and $I_{s a t}$ are used as fitting parameters. The red curve in figure 3.12 is the calculation of the integrated fluorescence signal from a homogeneous (top hat) intensity distribution, in which we have used the values of $C_{k i}$ and $I_{s a t}$ as found from the Gaussian fit.

It can be clearly seen that even in the saturation regime, the LIF signal generated with a Gaussian excitation profile is still dependent on the laser excitation energy, though dramatically less than in the linear regime. Due to the relative small slope, the shot-to-shot fluctuations in the output power of the excitation laser of $10 \%$ (section 3.3.1) become reduced to values between 1 and $2 \%$ in LIF signal power. Therefore, measurements in the saturation regime in spite of the one-dimensionally non-uniform excitation still approximately retain all advantages discussed in section 2.2 .

It should be noted that for a qualitative comparison of densities, all LIF measurements on a specific species in the plasma should be carried out at the same laser excitation energy in order to allow a comparison of densities. For instance, if a first measurement is done at an excitation laser energy of $40 \mu \mathrm{J}$ and a second is done with $80 \mu \mathrm{J}$, both measurements would be within the saturation regime of LIF, so for each measurement individually the LIF signal remains proportional to the species density. However, in this given example, the same species density would give a $20 \%$ lower LIF signal for an excitation laser energy of $40 \mu \mathrm{J}$ as compared to $80 \mu \mathrm{J}$. Therefore, a quantitative comparison of different measurements of a specific species is only possible if these are carried out at the same laser excitation energy.

An additional requirement of quantitative comparison of densities of different species is that for all LIF measurements done in the saturation regime the detection system is calibrated for each species separately. For such a calibration, absorption measurements can be used, which will be discussed in the next section. 


\subsection{Absorption spectroscopy}

As discussed at the end of section 2.2, LIF measurements in the saturation regime allow to generate a relative density map of species in the PLD plasma plume. However, a calibration factor is needed to convert the relative density map into an absolute density map. In section 2.3 , we have shown that absorption spectroscopy (AS) can be used to provide the corresponding calibration factor by measuring the spatially integrated density of particles along the LIF excitation beam path and linking this to the spatial distribution. To obtain the integrated density of particles present in the LIF beam with AS, we recall equation 2.28:

$$
\int N_{i}(x) d x=\frac{\int \tau(v) d v}{\pi r_{0} c f_{i k}},
$$

where $\int N_{i}(x) d x$ is the integrated number of particles in the beam path, $\tau$ is the optical thickness, $\left[\ln \left(I / I_{0}\right)\right]$, where $I_{0}$ is the incident and $I$ is the transmitted probe laser intensity, and $f_{i k}$ is the absorption oscillator strength of the excitation transition being used. The spectrally integrated optical depth is defined as

$$
\int \tau(v) d v \equiv \int \ln \left(\frac{I_{0}}{I}\right) d v .
$$

The optical depth is measured by spectrally scanning the dye laser wavelength (or its second harmonic) over the absorption line and measuring the laser intensity before $\left(I_{0}\right)$ and after $(I)$ passing through the plasma plume. To measure the incident and transmitted probe intensities versus wavelength, we built the setup as shown in figure 3.13.

To measure the incident laser beam intensity both before and after traversing the plasma plume, a broadband UV beam splitter is placed just in front of the input window of the PLD chamber, as shown in figure 3.13. The beam splitter reflects about half of the laser beam intensity to a large-area UV enhanced silicon photodiode, PD1 (Edmund Optics, UV Enhanced Response Silicon Detector, 50.0 $\mathrm{mm}^{2}$ ). The PD detects the reflected laser input power which scales proportional to the laser input intensity $I_{0}$. The beam splitter transmits approximately the other half of the beam intensity to another PD (PD2) that is placed on the other side of the PLD chamber, where the laser beam exits the chamber through a window. The laser power is measured by PD2 after traversing the plasma plume, which yields a signal that is proportional to the intensity as well $(I)$. A reverse bias is applied to both PDs to increase the detection speed. This is necessary for assuring that scattering light from the the target ablation laser, which is also detected by the PDs, does not overlap with the AS signal. Despite the reverse bias, the PDs are still very slow compared to the pulse length of the probe beam, having a response time (signal fall-time) over several microseconds, as compared to the probe beam which has a pulse length in the nanosecond-range. Due to the relatively long response times of the photodiodes, the entire probe pulse is absorbed by the PDs before a 


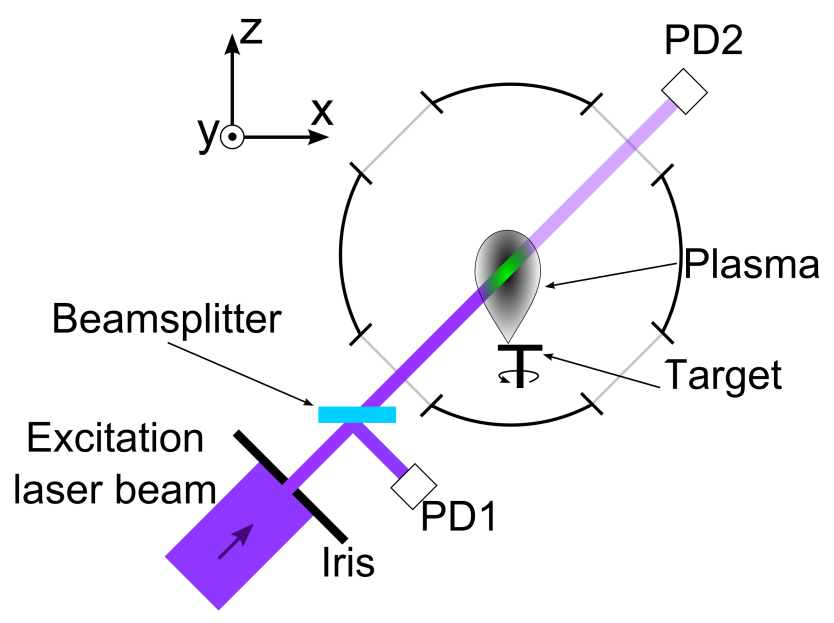

Figure 3.13: Schematic of absorption spectroscopy setup build at the PLD chamber. A small section of the excitation laser sheet is selected using an iris. A beam splitter reflects part of the incident beam onto a photodiode (PD1), and transmits light into the PLD chamber. After passing through the plasma plume, the laser light intensity is detected by another photodiode (PD2).

voltage response is measured in this case. The peak signal of response of the PD becomes proportional to the integrated probe pulse energy.

For the AS measurement to be reliable, two important requirements must be met: firstly, the PDs must have a linear response, and, secondly, a saturation of the absorption must be avoided, meaning that the measurements are to be carried out in the linear regime for LIF.

To check the linearity of the PDs, a calibration measurement (see figure 3.14) was carried out by attenuating the probe laser beam using calibrated OD filters. Figure 3.14 shows the results of this measurement, plotting the peak voltage of the output of the PDs as function of the input pulse energy, along with linear fit.

This measurement shows that for measured signals below $500 \mathrm{mV}$, the PDs display a linear response. In order to remain in this regime, we have attenuated the LIF probe beam to set the maximum signal (when no plasma is present) approximately below $500 \mathrm{mV}$. This is done by placing neutral density filters with a combined OD of at least 2.3 in the beam path in front of the LIF beam expander.

Secondly, it is to be checked whether the maximum pulse energy in the linear response range of the PDs also lies in the linear regime for LIF. As discussed in section 2.3, in this regime the absorption of the LIF excitation beam lies in the linear regime as well. In this case, the population density $N_{i}$ should not noticeably depend on the input pulse energy. In saturation, all LIF measurements are done with the laser excitation pulse energy entering the PLD chamber set to $80 \mu \mathrm{J}$. It can be calculated that using the combination of an OD filter of 2.3 with the approximate $50 \%$ beam splitter, as was selected for avoiding photodiode signal saturation, re- 


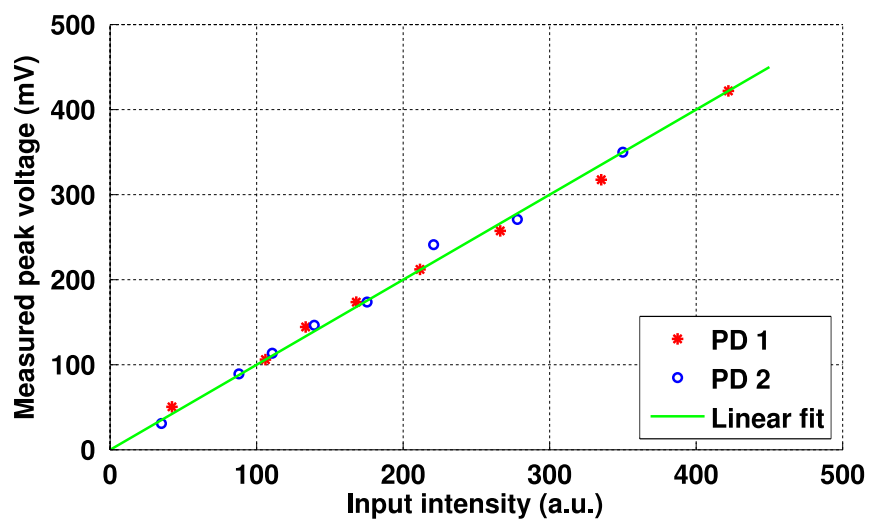

Figure 3.14: Linearity measurement of the photodiodes used in the absorption spectroscopy measurements. The plot shows the measured peak voltage as produced by the photodiodes as function of the input energy. In green is plotted a linear fit.

sults in a maximum pulse energy of $0.2 \mu \mathrm{J}$ for the AS measurements. Comparing this value with the inset of figure 3.12, restricting the maximum pulse energy to a value of $0.2 \mu \mathrm{J}$ places the absorption well within the linear regime, meaning that both requirements for linear AS are met.

In figure 3.15 we show an example of a measurement of the absorption spectrum of the $294.2 \mathrm{~nm}$ transition of neutral Ti at a $16 \mu$ s delay after target ablation in a background of 0.1 mbar Ar gas. From the ratio of the incident (green circles) and transmitted (red circles) probe laser intensities passing through the plasma the optical thickness can be calculated.

The measurement signals are normalized to their respective intensities at zero detuning without a plasma present. Small fluctuations in the averaged input laser intensity, $I_{0}$, and the transmitted intensity, $I$, are caused by the shot-to-shot fluctuations of the laser output power, even after averaging over 20 shots. However, since the optical thickness in the linear regime is dependent only on the ratio between incident and transmitted laser intensities, and not their absolute values, these fluctuations are expected to have no influence on the optical thickness calculations.

The laser intensity transmitted through the plasma ( $I$, red circles in figure 3.15) shows a clear dip when scanning over the absorption line, indicating a strong absorption of the light by the Ti present in the plasma plume. Figure 3.15 also shows the optical thickness (black cross symbols) calculated from the ratio of the incident and transmitted intensities according to equation 2.22. Selecting a fit curve with a Gaussian shape (blue line) was done to represent that Doppler broadening is expected to dominate the bandwidth of the absorption line (see section 2.4), as compared to the finite laser bandwidth. It can be seen that the fit matches the experimental data reasonably well.

From the spectral integral of the optical thickness we obtain the velocityintegrated particle number density (along the beam path) necessary for calibra- 


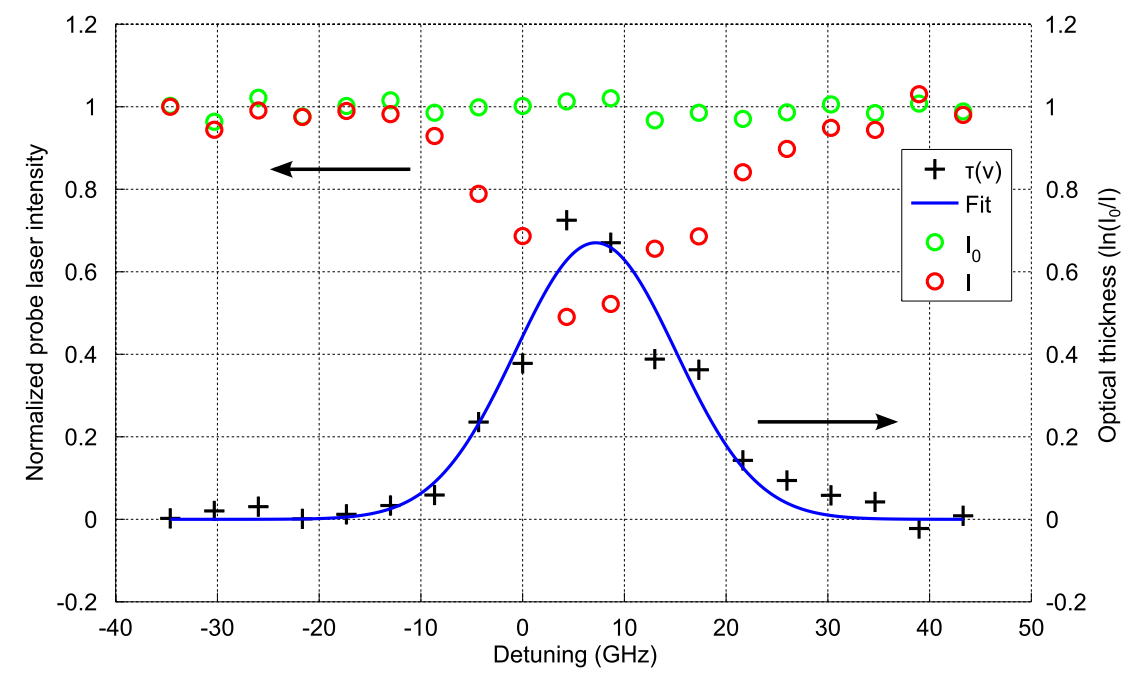

Figure 3.15: Normalized spectral shapes of incident (green circles) and transmitted (red circles) probe laser beam intensity for the $294.2 \mathrm{~nm}$ absorption line of neutral Ti for a delay of $16 \mu \mathrm{s}$ after target ablation in $0.1 \mathrm{mbar}$ Ar gas background. The measurements are normalized to their respective intensities at zero $\mathrm{GHz}$ detuning without an ablation plasma present. The optical thickness of the absorption line (black cross symbols) is calculated from the ratio of incident and transmitted probe laser intensities as a function of the detuning from the approximate absorption line center. The Gaussian fit to the data points is shown as the blue curve.

tion of the absolute number density. The Gaussian fit shows a FWHM of $16.8 \mathrm{GHz}$, which corresponds to an absorption line width of $15.8 \mathrm{GHz}$ after deconvolution with the laser linewidth. Interestingly, this is significantly broader than the 7.5 $\mathrm{GHz}$ that was calculated for this Ti line at the expected temperature of $5.000 \mathrm{~K}$ (see section 2.4). One possible explanation for the difference in linewidth is that the plasma plume is still expanding at velocities of up to $1500 \mathrm{~m} / \mathrm{s}$. Such a velocity translates into a Doppler shift of approximately $5 \mathrm{GHz}$. Because the plasma is expanding towards the excitation laser beam propagation at the edge where the beam enters the plasma, and also expands away from the excitation laser at the edge where the beam exits the plasma, this effect would cause an additional broadening to the absorption spectrum. The broadening of the absorption spectrum due to rapid expansion of the plasma plume is supported by measurements done by Williamson et al. [8], who observed an increase in absorption linewidth for plasmas expanding in vacuum as compared to expansion in 25 mTorr, and attributed this increase to the modified velocity distribution of the plasma plume caused by expansion.

The resolution of the AS measurements is primarily determined by the stepsize by which we can reliably tune the output wavelength of the dye laser, which 
was determined to be $1.25 \mathrm{pm} \pm 20 \%$. This allows us to determine the absorption profile with a higher precision compared to previous AS measurements of PLD plasmas, such as Williamson et al. [8] (resolution of $3 \mathrm{pm}$ ) and Dutouquet et al. [3] (resolution of $17 \mathrm{pm}$ ).

The apparent shift of the line center by $7 \mathrm{GHz}$ with respect to nominally zero detuning is caused by a hysteresis in the wavelength selection mechanism of the dye laser. When switching from increasing the output wavelength to decreasing the output wavelength, the dial indicating the output wavelength could be tuned by approximately $7 \mathrm{GHz}$ without changing the output wavelength.

\subsection{Synchronization}

To ensure reliable LIF and AS measurements, all laser and detection systems involved must be synchronized to each other, which includes the ablation and LIF excitation lasers, the PDs and the iCCD camera. Two of these systems are run at specific repetition rates. For optimal performance, the Nd:YAG laser has to be operated at $10 \mathrm{~Hz}$ (see section 3.3.1). The iCCD camera, on the other hand, can take measurements only at a maximum repetition rate of $2 \mathrm{~Hz}$, due to the time required to process the captured images in the camera, transfer the captured data from the camera to the PC and store it. In general, the iCCD is operated at its maximum rate of $2 \mathrm{~Hz}$. For a precise synchronization, it also has to be taken into account that the laser systems and the iCCD camera exhibit individual delayed responses to their trigger signals. To adjust the various trigger signals to a proper timing, we have built a chain of delay generators driven by a master trigger at $10 \mathrm{~Hz}$. A schematic of this setup is shown in figure 3.16, and an overview of delays, triggers and optical outputs is shown in figure 3.17.

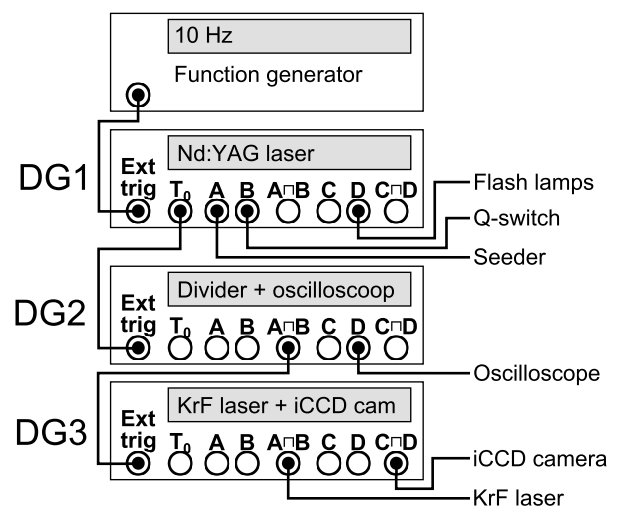

Figure 3.16: Schematic of the delay generators (DG) that are used to form a trigger chain for the proper timing of all systems of the setup.

The TTL output of a digital function generator (MCR Systems WG-1220) is used as the master trigger and is set to $10 \mathrm{~Hz}$, which corresponds to the optimum 


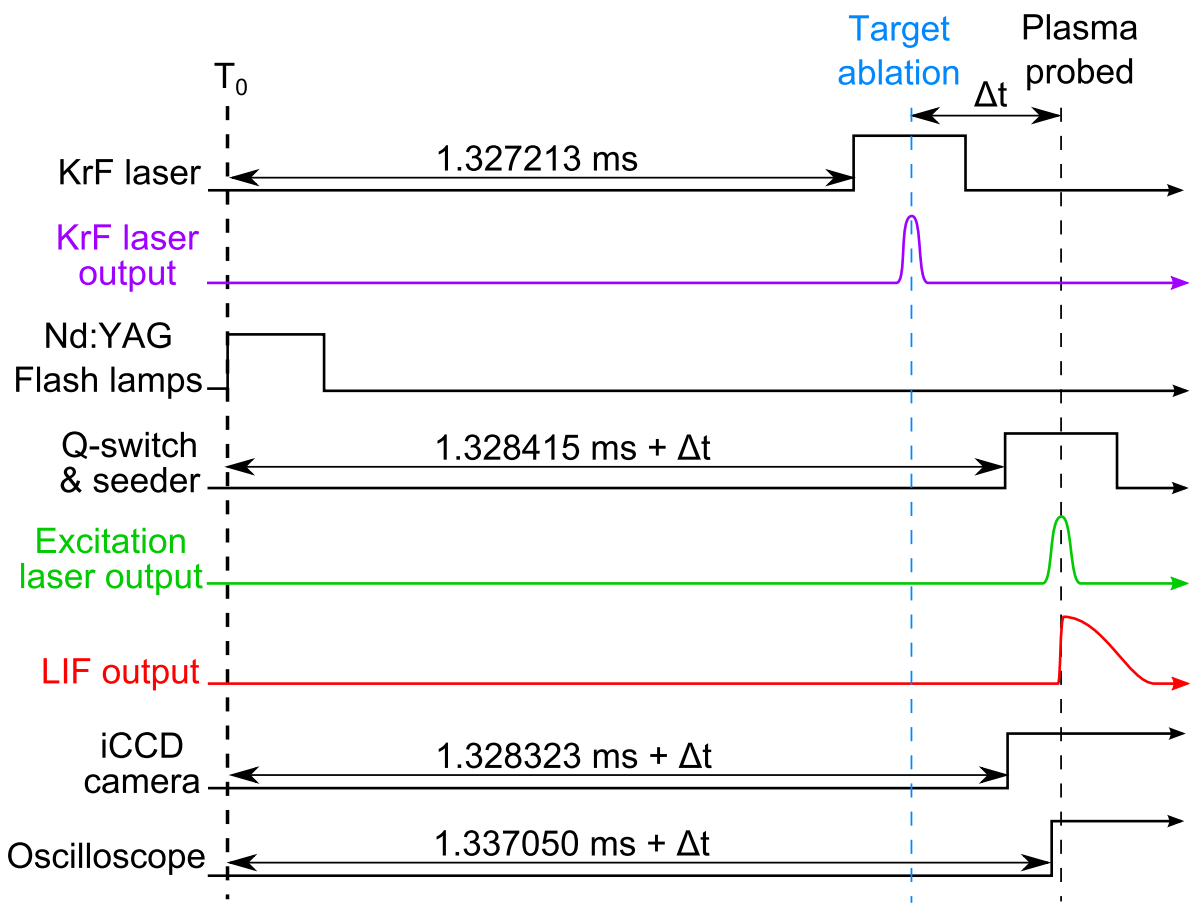

Figure 3.17: Overview of the timing of all triggers and optical outputs. At $T_{0}$, the $N d$ :YAG flash lamps are triggered. If $\Delta t$ is set to 0 , all other triggers are set to let target ablation (purple trace and blue dashed line), coincide in time with plasma probing and LIF detection (green and red traces, respectively, and black dashed line). By increasing $\Delta t$, the plasma can be probed at an adjustable delay time of $\Delta t$ with regard to target ablation.

repetition rate of the Nd:YAG laser. At $T_{0}$, a TTL pulse from DG1 triggers the flash lamps of the Nd:YAG laser. The delay between the flash lamps and the Q-switch is optimized for maximum output energy. If $\Delta t$ is set to 0 , all the trigger delays are set to have the ablation pulse (purple line in figure 3.17), the excitation pulse (green line), subsequent LIF output (red line) and detection by the iCCD camera and PDs, as observed with an oscilloscope, overlap. If a non-zero value is chosen for $\Delta t$, all systems except the $\mathrm{KrF}$ laser are delayed by $\Delta t$, allowing to probe the plasma plume at an adjustable delay time, $\Delta t$, after target ablation.

To allow the iCCD camera to measure at its maximum rate of $2 \mathrm{~Hz}$ while the LIF laser system operates at $10 \mathrm{~Hz}$, one of the delay generators (DG2) is set up as a 'divider-by-n' of the LIF excitation laser repetition rate. This is achieved by setting one of the delays to a value of $n$-times $100 \mathrm{~ms}$, which slows the data acquisition rate to a value of $10 / n \mathrm{~Hz}$. Generally, DG2 is set to $1 \mathrm{~Hz}$ during optical alignment of the setup and to $2 \mathrm{~Hz}$ during LIF and AS measurements. DG2 drives DG3 which triggers both the KrF laser used for target ablation and the iCCD camera. The 
$\mathrm{KrF}$ laser is run intentionally in synchronization with the iCCD camera, to avoid unnecessary ablation of the target in between measurements.

\subsection{Summary}

In this chapter we have discussed the experimental setup that is designed and built for the purpose of applying in-situ LIF and AS on PLD plasmas. To allow for optical accessibility of the plasma, a custom PLD chamber is built, as discussed in section 3.1. Ablation of the target inside the PLD chamber is carried out using a $\mathrm{KrF}$ excimer laser, of which the output beam is spatially filtered to ensure homogeneous ablation (section 3.2). The generation and detection of LIF requires a setup comprising of many different elements, which are discussed in section 3.3. An optically pumped dye laser generates narrowband light pulses of several ns in time, and has a wavelength that is tunable over a very broad spectral region (540 nm to 900 $\mathrm{nm}$ ). To extend the range of available excitation wavelengths, a second harmonic generation setup is added, capable of converting the output of the dye laser into the UV region of $270 \mathrm{~nm}$ to $450 \mathrm{~nm}$. The excitation laser beam is shaped into a thin light sheet to allow the probing of a cross section of the plasma plume. For the detection of LIF we use an intensified CCD camera, equipped with a custom zoom lens system.

To verify that all measurements are done in their proper regimes, i.e., LIF in the saturation regime and AS in the linear regime, we have measured the LIF signal as function of the excitation energy in section 3.4. These measurements agreed well with the theory discussed in chapter 2, although the influence of the spatial distribution of the excitation beam had to be taken into account. In section 3.5 we discuss the setup and experimental challenges for applying AS to PLD plasmas. All of the lasers and detection systems are synchronized using a chain of delay generators driven by a master trigger running at $10 \mathrm{~Hz}$. The delay time between target ablation and probing of the plasma plume can be manually set to any value between $0 \mathrm{~ns}$ and several $\mathrm{ms}$ in steps of a ns. The plasma probe frequency is limited to a maximum of $2 \mathrm{~Hz}$ by the data acquisition time of the iCCD camera, but can otherwise be set to any required frequency. 


\section{References}

${ }^{1}$ T. Okada and M. Maeda, "Laser spectroscopic studies of pulsed-laser deposition process for high- $\mathrm{T}_{c}$ thin films", Materials Science \& Engineering, B: Solid-State Materials for Advanced Technology 47, 64 (1997).

${ }^{2}$ D. Geohegan, A. Puretzky, and D. Rader, "Gas-phase nanoparticle formation and transport during pulsed laser deposition of $\mathrm{Y}_{1} \mathrm{Ba}_{2} \mathrm{Cu}_{3} \mathrm{O}_{7-d}$ ", Applied Physics Letters 74, 3788 (1999).

${ }^{3}$ C. Dutouquet and J. Hermann, "Laser-induced fluorescence probing during pulsed-laser ablation for three-dimensional number density mapping of plasma species", Journal of Physics D: Applied Physics 34, 3356-3363 (2001).

${ }^{4}$ R. Al-Wazzan, C. Lewis, and T. Morrow, "A technique for mapping three-dimensional number densities of species in laser produced plumes", Review of Scientific Instruments 67, 85-88 (1996).

${ }^{5}$ R. Eason, ed., Pulsed laser deposition of thin films (John Wiley \& Sons, Inc., 2007).

${ }^{6}$ L. P. Division, Quanta-ray pdl-3 pulsed dye laser intstruction manual, SpectraPhysics (1989).

${ }^{7}$ A. Smith, Snlo software, http://www.as-photonics.com/snlo.

${ }^{8}$ T. Williamson, G. Martin, A. El-Astal, A. Al-Khateeb, I. Weaver, D. Riley, M. Lamb, T. Morrow, and C. Lewis, "An investigation of neutral and ion number densities within laser-produced titanium plasmas in vacuum and ambient environments", Applied Physics A 69, S859-S863 (1999). 


\section{SPATIAL AND TEMPORAL MAPPING OF AL AND ALO DURING OXIDATION IN PULSED LASER ABLATION OF $\mathrm{LAALO}_{3}$}

We present the first spatio-temporal mapping of two simultaneously present species in a plasma used for pulsed laser deposition. We apply laser-induced fluorescence spectroscopy (LIF) to map ground state populations of Al and AlO in plasma plumes generated by ablation of $\mathrm{LaAlO}_{3}$ in an $\mathrm{O}_{2}$ or Ar atmosphere. Around a specific distance from the target, we observe Al vanish simultaneously with the turnup of AlO. This can be assigned to the oxidation of Al in the background atmosphere occurring after a sufficient slowing of the expanding Al front. This is consistent with the absence of such effects when argon is taken as background. A quantitative evaluation of data promises to reveal important dynamical reaction and deposition parameters for identification of the origin of specific properties of PLD grown films such as 2-dimensional conductive interfaces.

\section{Introduction}

Pulsed Laser Deposition (PLD) is a versatile technique to grow thin films through the deposition of laser ablated material. It allows for the growth of complex materials, like high-temperature superconductors, oxide based ferroelectrics and piezoelectrics [1]. Recently, also film stacks with atomic sharp interfaces are grown which exhibit intriguing properties. One such structure receiving significant attention lately is a thin film oxide heterostructure formed by deposition of $\mathrm{LaAlO}_{3}$ (LAO) on a $\mathrm{SrTiO}_{3}$ (STO) substrate. Ohtomo et al. discovered a high concentration of charge carriers with high mobility confined to the interface of LAO and STO, referred to as a two-dimensional electron gas (2DEG) [2]. These 2DEGs have since been extensively researched [3] and several additional features of the interface 
have been observed, including superconductivity [4] and magnetism [5].

To explain the high conductivity at the LAO/STO interface, several possible mechanisms have been proposed. These include the creation of oxygen vacancies in the STO substrate resulting from the diffusion of oxygen from the STO to the LAO film which is not fully oxidized. Kalabukhov et al. showed that indeed oxygen vacancies were formed when an LAO film was grown at low oxygen pressures $\left(10^{-6}\right.$ mbar) [6] caused possibly by an incomplete oxidation of La and $\mathrm{Al}$ in the plasma plume $[7,8]$. In search for a better understanding and control of the 2DEG, the influence of the PLD parameters, such as background gas and pressure, and laser fluence, on the conductivity at the interface has been studied extensively [3].

However, this empiric approach does not provide direct insight in the oxidation state of the material flux propagating towards the substrate and the influence on the growth of the LAO film. For this, different methods are needed that allow a direct spatial and temporal mapping of the density and flow of the involved species towards the substrate. Of central interest is that chemical reactions become displayed and quantified, such as the dynamics of oxidation, which requires the simultaneous mapping of at least two species.

In an attempt to relate film growth to the material flux in the plasma plume, optical emission spectroscopy (OES) has been used. However, only excited particles that also fluoresce are accessible with this diagnostic method [9-11]. This is a large drawback, because the populations in the excited states are orders of magnitude lower than the ground state populations when the plume has expanded close to the substrate [12]. This means that signals are weak at times beyond the fluorescence lifetime and only qualitative and limited information can be gained. However, laser-induced fluorescence (LIF) allows a direct spatial and temporal mapping of the density and flow of the involved species towards the substrate [13-15].

In this paper, we report for the first time the spatial and temporal mapping of two species simultaneously, namely aluminum and aluminum oxide, which are related through a chemical reaction in the pulsed laser ablation plasma of $\mathrm{LaAlO}_{3}$.

\subsection{Experimental setup and methodology}

The LIF measurements are carried out in a custom built PLD chamber (Twente Solid State Technology), designed specifically for the optical diagnostics of the plasma plume. A schematic view of the setup is shown in Fig. 4.1. The plasma plume is generated by irradiating the target with laser pulses generated by a $\mathrm{KrF}$ excimer laser (248 nm, $30 \mathrm{~ns}$ duration FWHM, operating at $2 \mathrm{~Hz}$ ). Using a mask placed in the $\mathrm{KrF}$ laser beam, a spatially uniform beam, with a cross section of $4 \times 15 \mathrm{~mm}^{2}$ and demagnified by a factor of 6.21 , is incident onto the target at a $45^{\circ}$ angle of incidence. This results in a laser spot of $0.91 \times 2.42 \mathrm{~mm}^{2}$. The laser fluence is kept at $1.3 \pm 10 \% \mathrm{~J} / \mathrm{cm}^{2}$ during all measurements. To prevent local heating and drilling, the LAO ablation targets are mounted on a rotating holder. The deposition chamber, with a base pressure of $2 \cdot 10^{-7} \mathrm{mbar}$, is filled with either oxygen gas (99.9999\%) or argon gas (99.999\%). 


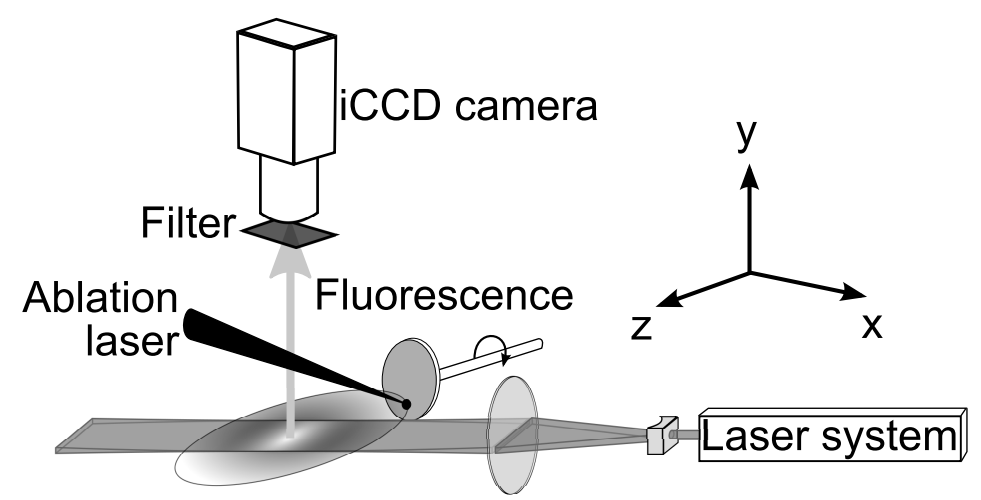

Figure 4.1: Schematic of the setup. The ablation laser is a KrF laser. The laser system used for the LIF measurements consists of a Nd:YAG laser pumped dye laser with second harmonic generation. The combination of a cylindrical and spherical lens is used to both expand and focus the laser beam in the plane of expansion of the plasma plume. The LIF sheet is set at a $45^{\circ}$ angle with the z-axis. Fluorescence is detected through appropriate optical filters with an intensified CCD camera.

Detection of both the spontaneous emission, by which we designate the emission of the hot plasma plume, and the LIF is done with an intensified charge coupled device (iCCD) camera, equipped with a fused silica imaging system to allow for UV imaging. During the LIF detection of AlO, a colored glass band-pass filter $(275-375 \mathrm{~nm})$ is used to suppress most of the spontaneous emission. For Al detection, a more narrowband interference filter of $307 \pm 5 \mathrm{~nm}$ is used. To further reduce the spontaneous emission in the LIF measurements, a background subtraction (a measurement with the LIF beam turned off) is applied to all LIF measurements.

The gating time of the camera intensifier is used as shutter. The gating time for the LIF measurements is set to $44 \mathrm{~ns}$, which is found to be the shortest time to still include almost all of the fluorescent decay. For the spontaneous emission measurements, the gating time is adjusted for maximum signal without saturating the camera, which is $120 \mathrm{~ns}$ at the longest delay times after the target ablation. All LIF images are averaged over 20 to 30 shots, depending on the signal strength, to increase the signal-to-noise ratio. The LIF lasers and iCCD camera are synchronized to the ablation laser operating at $2 \mathrm{~Hz}$.

To image the neutral aluminum, we use an excitation of the atomic ground state transition at $308.2153 \mathrm{~nm}\left(3 s^{2} 3 p^{2} P^{\circ} 1 / 2 \rightarrow 3 s^{2} 3 d^{2} D^{3} / 2\right)$. Relaxation occurs to the $3 s^{2} 3 p^{2} P^{\circ} 3 / 2$ state via fluorescence at $309.2839 \mathrm{~nm}$ [16]. The excitation of aluminum oxide is done using the $X^{2} \Sigma \rightarrow C^{2} \Pi$ transition at $302.16 \mathrm{~nm}$ and detecting the red-shifted fluorescence between 303 and $306 \mathrm{~nm}$ [17].

The excitation wavelengths are generated by a dye laser pumped with the second harmonic (532 nm) output of a Q-switched Nd:YAG laser (7 ns FWHM). By frequency doubling the output of Rhodamine 640, both UV excitation wavelengths ( 6 ns FWHM, 30 pm bandwidth, $0.14 \mathrm{~mJ}$ ) can be produced with tuning of the dye 
laser. To image a cross section of the ablation plume, the laser beam is transformed into a thin sheet. This is done with the combination of a cylindrical and a spherical lens, allowing for expansion in the xz-plane to a width of $35 \mathrm{~mm}$ (50 mm along the $\mathrm{z}$-axis due to the $45^{\circ}$ incidence angle), while the sheet is focused to approximately $0.5 \mathrm{~mm}$ thickness. Both lasers and the camera are triggered using a cascaded chain of delay generators.

\subsection{Results}

To investigate the oxidation of the plasma plume, we use LIF to map the spatiotemporal distribution of neutral aluminum atoms as well as neutral aluminum oxide molecules in the ground state. The location and density of both species is mapped in both oxygen and argon backgrounds.

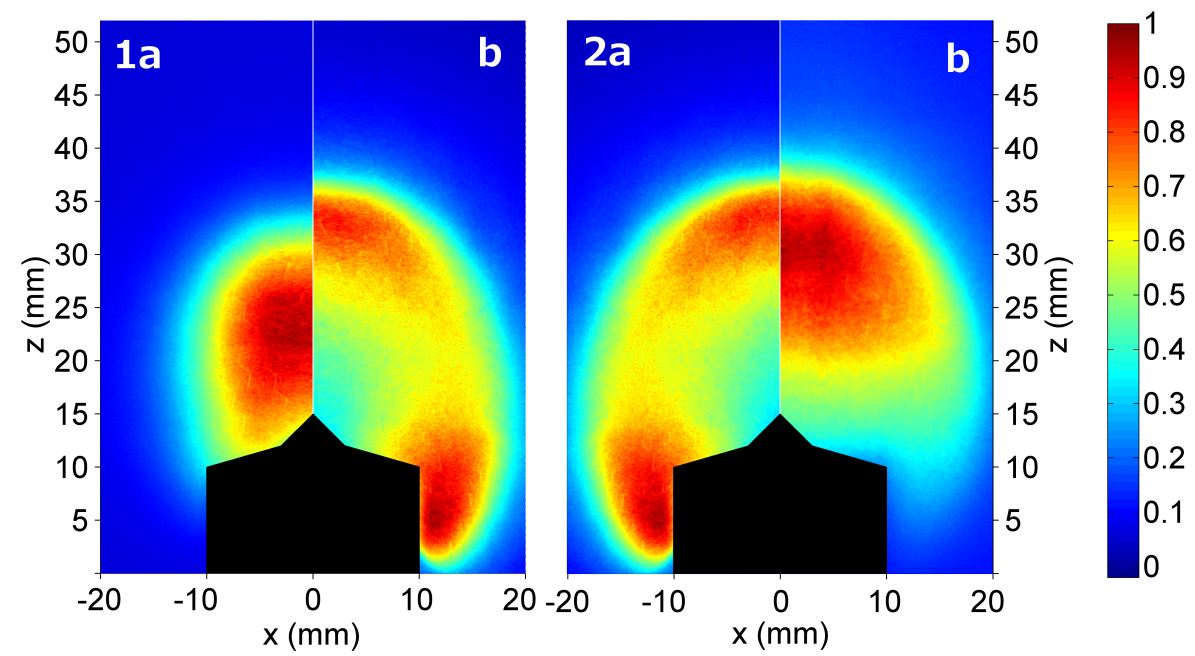

Figure 4.2: Images of LIF (1a, $1 b \& 2 a$ ) and of spontaneous emission (2b) of an LAO plasma plume versus the $a$ and $z$ cross section coordinates of the plume as in Fig. 1. $Z=O$ corresponds to the surface of the ablation target. All measurements are done in $1 \cdot 10^{-1}$ mbar $\mathrm{O}_{2}$ background pressure and at $10 \mu$ s delay from ablation. 1a) LIF of $\mathrm{Al}$ in $\mathrm{O}_{2} .1 \mathrm{~b} \& 2 \mathrm{a}$ ) LIF of $\mathrm{AlO}$ in $\mathrm{O}_{2}$. 2b) Spontaneous emission in $\mathrm{O}_{2}$. The lower part of the image is blackened because in this region the laser-induced fluorescence and spontaneous emission is obstructed by the target holder which is located between the target and the iCCD camera.

Figure 4.2 shows a typical result of these measurements, at a delay of $10 \mu \mathrm{s}$ between ablation and detection. For a direct comparison of the $\mathrm{Al}$ vs. AlO distribution (Fig 4.2.1) and the AlO LIF vs. the spontaneous emission (Fig. 4.2.2), respectively, we have plotted next to each other the according half-images. Since in each single measurement the maximum is normalized to unity, the density maps cannot be 
compared in terms of absolute density. However, the relative distributions are well quantified.

In Fig. 4.2.1 the LIF of $\mathrm{Al}$ is compared to that of AlO. At the delay of $10 \mu \mathrm{s}$, the plasma plume has expanded several tens of mm's and the velocity of the $\mathrm{Al}$ atoms is reduced such by collisions with the $\mathrm{O}_{2}$ background that they react. $\mathrm{AlO}$ is preferentially formed at the edges of the plume where the atoms are in direct contact with the $\mathrm{O}_{2}$.

Figure 4.2.2 shows the LIF image of AlO next to the spontaneous emission which is measured without any filtering. The density distribution of AlO (Fig. 4.2.2a) is not represented in the image of the spontaneous emission (Fig. 4.2.2b), nor is the density distribution of $\mathrm{Al}$ (Fig. 4.2.1b). This underlines that OES is not suited for the mapping of populations in the plasma plume.

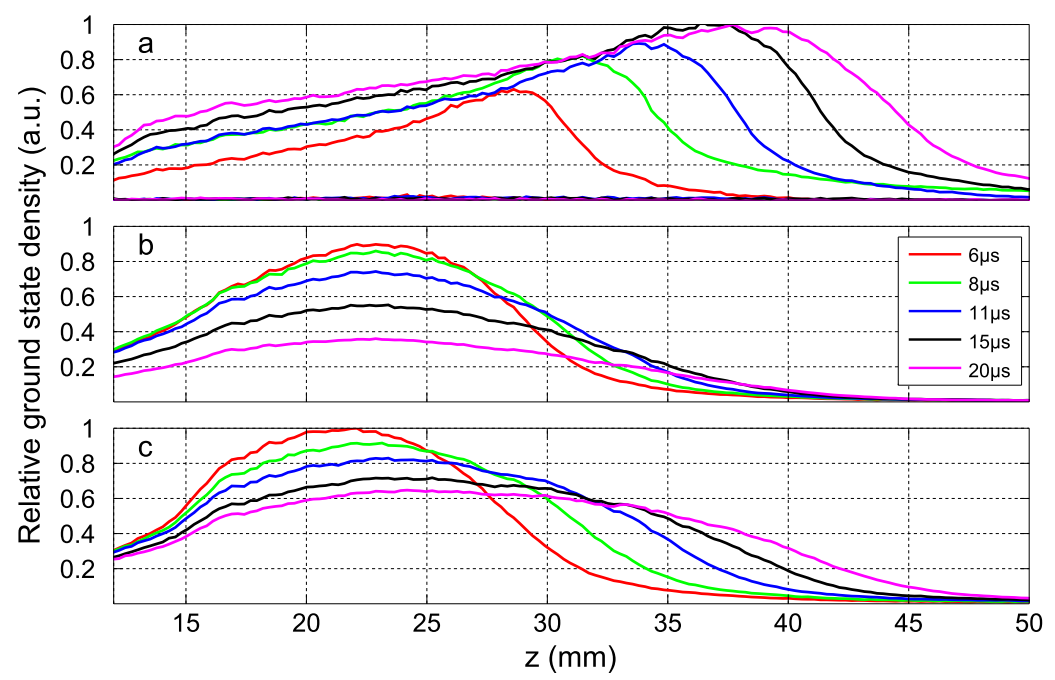

Figure 4.3: Relative ground state population densities of $\mathrm{AlO}(\mathrm{a})$ and of $\mathrm{Al}(\mathrm{b} \& \mathrm{c}$ ) along the propagation axis as function of the distance from the target at different delay times. Graphs a \& b represent measurements done in an $\mathrm{O}_{2}$ background, graph $\mathrm{c}$ is in Ar. All measurements are done in $1 \cdot 10^{-1}$ mbar background pressure. The relative densities of $A l$ in graphs $b$ \& $c$ can be compared.

The relative densities of $\mathrm{Al}$ and $\mathrm{AlO}$ in the ground state are presented in Fig. 4.3 as function of distance from the ablation target at different delay times from ablation and in different background gasses. Figure 4.3a shows that aluminum oxide is formed mostly at the front of the plume, where interaction with the background gas is strongest. Conversion of atomic aluminum to aluminum oxide can also be seen in the back of the plume, as the concentration of $\mathrm{Al}$ in Fig. 4.3b decreases in time, whereas the density of $\mathrm{AlO}$ increases (Fig. 4.3a). The absence of aluminum in the front of the plume suggests that all ground-state $\mathrm{Al}$ oxidizes in this region. 
The same measurements in $\mathrm{Ar}$ instead of $\mathrm{O}_{2}$ (Fig. 4.3c) show a clear difference in oxidation. More $\mathrm{Al}$ is present in $\mathrm{Ar}$ compared to $\mathrm{O}_{2}$ which spreads out more over the entire plume, including the front. AlO is not present in $\mathrm{Ar}$ in detectable quantities (plotted at the bottom of Fig. 4.3a).

Measurements have been done on $\mathrm{Al}$ and AlO LIF in lower background pressures $\left(1 \cdot 10^{-2}\right.$ and $\left.1 \cdot 10^{-3} \mathrm{mbar}\right)$, but our setup was unable to detect AlO due to the LIF signal being too small to measure. The spontaneous emission that is transmitted by the detection filter, which increased at lower background pressures, was one of the reasons for the decreasing detectability of AlO. However, we did see a similar absence of $\mathrm{Al}$ in the front of the plume in $\mathrm{O}_{2}$ compared to $\mathrm{Ar}$. This suggests that oxidation of $\mathrm{Al}$ is still taking place in the front of the plume.

\subsection{Discussion \& Conclusions}

We have applied laser-induced fluorescence to measure the relative ground state densities of aluminum and aluminum oxide in the plasma plume of pulsed laser ablated $\mathrm{LaAlO}_{3}$. The oxidation of aluminum has been mapped spatially and temporally in an oxygen background of $1 \cdot 10^{-1}$ mbar. Oxidation of $\mathrm{Al}$ in the front of the plume has been observed, where direct mixing of the plasma with the background gas takes place. Oxidation of ground state $\mathrm{Al}$ also takes place in the center of the plume, but at a lower rate. An increase in Al density together with the absence of $\mathrm{AlO}$ in Ar confirms that aluminum is indeed oxidizing. The absence of $\mathrm{Al}$ in the front of the plasma plume in $\mathrm{O}_{2}$ compared to Ar, which was visible at all three pressures $\left(1 \cdot 10^{-1}, 1 \cdot 10^{-2}\right.$ and $1 \cdot 10^{-3}$ mbar) suggest that oxidation still takes place at these settings, but at a lower rate. This would support the observations of increased oxygen vacancies in the boundary layer between STO and LAO at lower background pressures.

For future work we will increase the sensitivity of our setup to improve the AlO LIF measurements at lower oxygen pressures. We are currently working on an absolute calibration of the LIF via absorption spectroscopy, which will allow us to map densities and flows of absolute values. The latter is the key for comparison with plasma expansion models that are currently being developed. 


\section{References}

${ }^{1}$ R. Eason, ed., Pulsed laser deposition of thin films (John Wiley \& Sons, Inc., 2007).

${ }^{2} \mathrm{~A}$. Ohtomo and H. Hwang, "A high-mobility electron gas at the $\mathrm{LaAlO}_{3} / \mathrm{SrTiO}_{3}$ heterointerface", Nature 427, 6973 (2004).

${ }^{3}$ M. Huijben, A. Brinkman, G. Koster, G. Rijnders, H. Hilgenkamp, and D. Blank, "Structure-property relation of $\mathrm{SrTiO}_{3} / \mathrm{LaAlO}_{3}$ interfaces", Advanced Materials 21, 1665 (2009).

${ }^{4}$ N. Reyren, S. Thiel, A. Caviglia, L. F. Kourkoutis, G. Hammerl, C. Richter, C. Schneider, T. Kopp, A.-S. Rüetschi, D. Jaccard, M. Gabay, D. A. Muller, J.-M. Triscone, and J. Mannhart, "Superconducting interfaces between insulating oxides", Science 317, 1196 (2007).

${ }^{5}$ A. Brinkman, M. Huijben, M. V. Zalk, J. Huijben, U. Zeitler, J. Maan, W. V. D. Wiel, G. Rijnders, D. Blank, and H. Hilgenkamp, "Magnetic effects at the interface between non-magnetic oxides", Nature Materials 6, 493 (2007).

${ }^{6}$ A. Kalabukhov, R. Gunnarsson, J. Börjesson, E. Olsson, T. Claeson, and D. Winkler, "Effect of oxygen vacancies in the $\mathrm{SrTiO}_{3}$ substrate on the electrical properties of the $\mathrm{LaAlO}_{3} / \mathrm{SrTiO}_{3}$ interface", Physical Review B: Condensed Matter and Materials Physics 75, 121404 (2007).

${ }^{7}$ W. Siemons, G. Koster, H. Yamamoto, W. Harrison, G. Lucovsky, T. Geballe, D. Blank, and M. R. Beasley, "Origin of charge density at $\mathrm{LaAlO}_{3}$ on $\mathrm{SrTiO}_{3}$ heterointerfaces: possibility of intrinsic doping”, Physical Review Letters 98, 196802 (2007).

${ }^{8}$ G. Herranz, M. Basletić, M. Bibes, C. Carrétéro, E. Tafra, E. Jacquet, K. Bouzehouane, C. Deranlot, A. Hamzić, J.-M. Broto, A. Barthélémy, and A. Fert, "High mobility in $\mathrm{LaAlO}_{3} / \mathrm{SrTiO}_{3}$ heterostructures: origin, dimensionality, and perspectives", Physical Review Letters 98, 216803 (2007).

${ }^{9}$ C. Aruta, S. Amoruso, R. Bruzzese, X. Wang, D. Maccariello, F. M. Granozio, and U. S. di Uccio, "Pulsed laser deposition of $\mathrm{SrTiO}_{3} / \mathrm{LaGaO}_{3}$ and $\mathrm{SrTiO}_{3} / \mathrm{LaAlO}_{3}$ : plasma plume effects", Applied Physics Letters 97, 252105 (2010).

${ }^{10}$ A. Sambri, D. Cristensen, F. Trier, Y. Chen, S. Amoruso, N. Pryds, R. Bruzzese, and $\mathrm{X}$. Wang, "Plasma plume effects on the conductivity of amorphous- $\mathrm{LaAlO}_{3} / \mathrm{SrTiO}_{3}$ interfaces grown by pulsed laser deposition in $\mathrm{O}_{2}$ and Ar", Applied Physics Letters 100, 231605 (2012).

${ }^{11}$ S. Amoruso, C. Aruta, P. Aurino, R. Bruzzese, X. Wang, F. M. Granozio, and U. S. di Uccio, "Oxygen background gas influence on pulsed laser deposition process of $\mathrm{LaAlO}_{3}$ and $\mathrm{LaGaO}_{3}$ ", Applied Surface Science 258, 9116 (2012).

${ }^{12}$ H. Döbele, T. Mosbach, K. Niemi, and V. S.-v. der Gathen, "Laser-induced fluorescence measurements of absolute atomic densities: concepts and limitations", Plasma Sources Science and Technology 14, S31 (2005). 
${ }^{13}$ T. Okada and M. Maeda, "Laser spectroscopic studies of pulsed-laser deposition process for high- $\mathrm{T}_{c}$ thin films", Materials Science \& Engineering, B: Solid-State Materials for Advanced Technology 47, 64 (1997).

${ }^{14}$ C. Dutouquet and J. Hermann, "Laser-induced fluorescence probing during pulsed-laser ablation for three-dimensional number density mapping of plasma species", Journal of Physics D: Applied Physics 34, 3356-3363 (2001).

${ }^{15}$ Y. Nakata, T. Okada, M. Maeda, S. Higuchib, and K. Ueda, "Effect of oxidation dynamics on the film characteristics of Ce:YIG thin films deposited by pulsedlaser deposition", Optics and Lasers in Engineering 44, 147 (2006).

${ }^{16}$ A. Kramida, Y. Ralchenko, J. Reader, and N. A. T. (2013), Nist atomic spectra database (ver. 5.1), National Institute of Standards and Technology, (2013) http: //physics.nist.gov/asd.

${ }^{17}$ R. Pearse and A. Gaydon, The identification of molecular spectra, 3rd ed. (Chapman and Hall, London, 1976). 


\section{INFLUENCE OF THE OXIDATION STATE OF $\mathrm{SRTIO}_{3}$ PLASMAS FOR STOICHIOMETRIC GROWTH OF PULSED LASER DEPOSITION FILMS IDENTIFIED BY LASER-INDUCED FLUORESCENCE}

By applying two-dimensional laser-induced fluorescence (LIF) on multiple plasma constituents, we are able to directly link the oxidation of plasma species in a $\mathrm{SrTiO}_{3}$ (STO) plasma for pulsed laser deposition (PLD) to the stoichiometry and quality of the thin films grown. With spatiotemporal LIF mapping of the plasma species in different background gas compositions, we find that Ti and Sr have to be fully oxidized for a stoichiometric growth of crystalline thin films, which gives new input for modeling surface growth, as well as provides additional control over the exact degree of stoichiometry of thin films.

\section{Introduction}

Pulsed laser deposition (PLD) has been demonstrated to be a superior technique for the growth of thin films of complex crystalline oxides such as to impose ferroelectricity [1], two-dimensional electron gases at heterointerfaces [2] or superconductivity at interfaces[3], among other applications [4]. It is widely accepted that PLD allows for stoichiometric transfer of complex materials, making it a very powerful and universal deposition technique. 
However, the growing of stoichiometric and defect-free films is far from trivial. Deposition is the result of complex spatiotemporal, plasma-chemical dynamics. This renders the growth of films highly dependent on the external growth parameters, such as ablation laser fluence $[5,6]$ and background gas pressure and composition [7], in manners that are not well understood. More specifically, the often stringent requirements concerning the external parameters to grow high-quality defect-free films indicate that the state in which plasma constituents arrive at the substrate (oxidation state, propagation speed and arrival time of different components) is of equal or even greater importance than just the stoichiometric transfer of materials. This can been seen, for instance, by the complex interplay between ablation fluence and target-substrate distance, and their combined influence on the thin film stoichiometry [8].

Using spatially and temporally resolved spectroscopy that can yield distributions of constituents of the plasma allows us to trace dynamical processes, such as the generation and propagation of oxidation fronts. Especially in chemically reactive plasmas, a direct link to the exact molecular state in which material arrives at the substrate can be the key to a deeper understanding of growing perfect crystalline thin films as cannot be gained via blind optimization of PLD parameters, but is required for a future upscaling of crystalline growth to larger areas.

Of particular interest is to investigate film growth in a well-known and wellinvestigated PLD reference system, $\mathrm{SrTiO}_{3}$ (STO) on a crystalline STO substrate. This allows sensitive X-ray diffraction measurements of the quality of growth, because even small defects in the film growth significantly change the lattice parameters.

Here we investigate the stoichiometry of the film growth while systematically varying the chemical background gas composition, changing the mixture of $\mathrm{O}_{2}$ and Ar, while maintaining the absolute total pressure constant. For an increasing fraction of $\mathrm{O}_{2}$, we observe a transition from non-stoichiometric to stoichiometric film growth. Specifically, the film quality shows a distinct leap to perfectly stoichiometric STO, which occurs at a certain $\mathrm{O}_{2}$ fraction $(\approx 60 \%)$ of a total pressure of 0.1 mbar. A total background pressure of $0.1 \mathrm{mbar}$ is chosen as the plasma expansion is no longer in the ballistic regime and has a strong interaction with the background gas, moderating the kinetic energy of the ablated species [7]. Argon and oxygen have a similar atomic weight and PLD plasmas exhibit very similar expansion dynamics in either $\mathrm{Ar}$ or $\mathrm{O}_{2}$ [9]. By mixing $\mathrm{Ar}$ and $\mathrm{O}_{2}$, we maintain a constant total pressure in all measurements with comparable gas dynamic processes, such as expansion, propagation speed and collision rates, for all gas mixtures. As the ablation laser fluence is kept constant for all measurements, changes in plasma composition will be primarily caused by a change in chemical processes and not by changing dynamics.

Via comparison with optically recorded $\mathrm{TiO}$ and $\mathrm{SrO}$ spatiotemporal distributions in the plasma we find that the oxidation state of $\mathrm{Ti}$ and $\mathrm{Sr}$ upon arrival at the substrate is correlated with the stoichiometry of growth. The optical measurements show that all Ti needs to be oxidized at the substrate location for achieving stoichiometric growth. Based on the SrO measurements, we conclude that this is 
also the case for Sr. These results provide a direct link and thus predictability for controlling the chemical plasma composition and subsequent growth of complex oxide thin films.

\subsection{Experimental setup and methodology}

The spatiotemporal mapping of the plasma constituents is carried out in a custom built PLD chamber, a detailed description of which can be found in a previous article of ours [10]. We use laser-induced fluorescence (LIF) instead of the more commonly used optical emission spectroscopy (OES) $[7,11,12]$, because OES is limited to the detection of excited particles that happen to spontaneously fluoresce. Another disadvantage of OES is that the excited state populations are orders of magnitude lower than the ground state populations when the plume has expanded close to the substrate[13]. In contrast, LIF enables us to excite and detect ground state species at freely selectable locations and times and in a chemically specific manner. This is of particular importance when selecting long delay times with respect to the moment of ablation, when the plume has cooled down and expanded over typically several centimeters towards the substrate [14-16].

Target ablation is done with laser pulses generated by a KrF excimer laser (248 $\mathrm{nm}, 30 \mathrm{~ns}$ duration FWHM, operating at $2 \mathrm{~Hz}$ ). A mask, placed in the $\mathrm{KrF}$ laser beam to select a spatially uniform beam, is imaged onto the target, resulting in a laser spot of $0.91 \times 2.42 \mathrm{~mm}^{2}$. Through control of the laser output energy, the laser fluence is kept at $1.3 \pm 10 \% \mathrm{~J} / \mathrm{cm}^{2}$ during all measurements. The UV excitation wavelengths for LIF in the range from 250 to $350 \mathrm{~nm}$ are generated by frequency doubling the output of a dye laser pumped with the second harmonic (532 nm) output of a Q-switched Nd:YAG laser (7 ns FWHM). The UV output, which has a pulse duration of $4 \mathrm{~ns}$ FWHM, a bandwidth of $8.1 \mathrm{pm}$ and $75 \mu \mathrm{J}$ per pulse, is transformed into a thin sheet in the plane of the forward propagation of the plasma plume from the ablation spot on the target to the center of the substrate. The sheet has an in-plane focus of approximately $0.4 \mathrm{~mm}$ thickness.

To image the neutral titanium distribution in the plasma plume, we use a transition from the atomic ground state $\left(3 d^{2} 4 s^{2} a^{3} F\right)$ to the $3 d^{2}\left({ }^{1} G\right) 4 s 4 p\left({ }^{3} P^{\circ}\right) v^{3} F^{\circ}$ state at $294.1995 \mathrm{~nm}$. Relaxation occurs to the $3 d^{3}\left({ }^{4} F\right) 4 s b^{3} F$ state via fluorescence at $445.3313 \mathrm{~nm}$ [17]. A narrowband interference filter with a bandpass of 437 to $447 \mathrm{~nm}$ transmits the LIF of Ti while suppressing the thermally induced spontaneous emission (SE) of the plasma. The excitation of titanium oxide is done using a $\mathrm{X}^{3} \Delta \rightarrow \mathrm{D}$ transition at $301.47 \mathrm{~nm}$ and detecting the red-shifted LIF between 301 and $310 \mathrm{~nm}$ [18]. Strontium oxide is excited using a $X^{1} \Sigma \rightarrow C^{1} \Sigma$ transition at $350.38 \mathrm{~nm}$ and detected using the red-shifted LIF from relaxation to various different rotational and vibrational states of $\mathrm{X}^{1} \Sigma$ [18]. For both TiO and SrO, the SE of the plasma is suppressed by a colored glass band-pass filter transmitting 275 to 375 $\mathrm{nm}$. A background subtraction using measurements with the LIF beam blocked is applied to all LIF measurements to remove any residual SE from the plasma that is transmitted by the band-pass filters. To reduce the influence of shot-to-shot fluc- 
tuations, all measurements are averaged over 30 shots. To improve the quality of the spatiotemporal mapping measurements, we decided to carry these out with the substrate removed, because in our setup with an oblique angle of the LIF beam, the substrate caused unwanted optical reflections. This modification avoids artifacts for the relevant observation times, until the plume arrives at the location of the substrate. The films deposited for the XRD measurements are grown on a substrate heated to $710^{\circ} \mathrm{C}$. The influence of substrate heating on the propagation dynamics of the plasma plume [19], present during deposition but absent during LIF measurements, is largely avoided by using laser substrate heating instead of the more commonly used resistive substrate heating. We performed comparative measurements of plume propagation with either a laser heated target at $710^{\circ} \mathrm{C}$ or at room temperature [20], which showed little difference, demonstrating the validity of the LIF measurements without heated substrate. In contrast, plume propagation with a resistive heated substrate at $710^{\circ} \mathrm{C}$ showed, compared to room temperature, large changes in propagation similar as described by Sambri et al [19].

\subsection{Results}

To investigate the chemical composition of the plasma plume and, more specifically, the effects of the background gas composition on the oxidation of the plasma constituents, we attempted to map the spatio-temporal distribution of all relevant and expectantly dominant plasma constituents which are $\mathrm{Ti}, \mathrm{TiO}, \mathrm{TiO}_{2}, \mathrm{Sr}$ and $\mathrm{SrO}$. The location, density and arrival time of these species is mapped in a background gas of which the total pressure is held constant at 0.1 mbar. The partial pressure of $\mathrm{O}_{2}$ is step-wise increased from $0 \%$ to $100 \%$ in order to step-wise increase the chemical reactivity.

The LIF signal of strontium atoms excited at either 293.18 and $689.25 \mathrm{~nm}$ could be detected, unfortunately, only close to the noise level and only at small delay times $(<10 \mu \mathrm{s})$ in pure Ar. Excitation at $460.73 \mathrm{~nm}$ might yield a higher fluorescence signal. However, generating this wavelength is not possible with our current setup. Similarly, measuring $\mathrm{TiO}_{2}$ densities proved difficult with excitation lines at $562.56 \mathrm{~nm}$ and $556.67 \mathrm{~nm}$ as described by Zhuang et. al. [21], due to strong background noise from scattering of the LIF laser, when using the LIF laser in the visible range.

To be able to relate the LIF signal to the material density, all excitation transitions used are driven into saturation. In saturation, a variation in laser power, for example pulse-to-pulse fluctuations or spatial inhomogeneities, will not result in variations of the LIF signal. Thereby, the LIF signal becomes proportional to the density of the excited species. However, since the measurements are not calibrated on an absolute scale, the density of one species can not be absolutely compared to that of another species.

Figure 5.1 shows typical results of distribution measurements for a crosssection of the plasma plume in the propagation plane from the target $(\mathrm{z}=\mathrm{x}=$ $0 \mathrm{~mm}$ ) to where the substrate is positioned during growth experiments $(\mathrm{x}=0 \mathrm{~mm}$, 
(a)

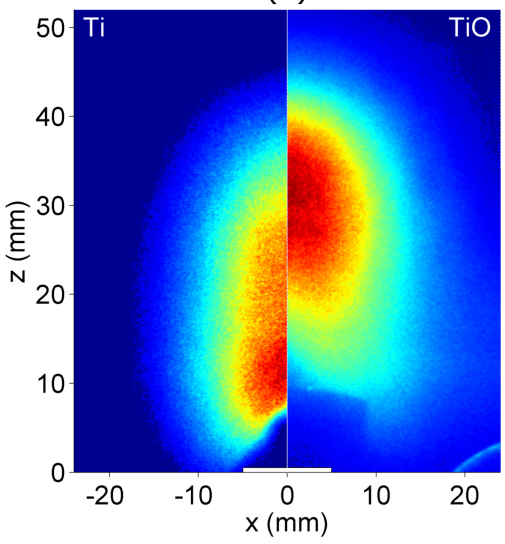

(b)

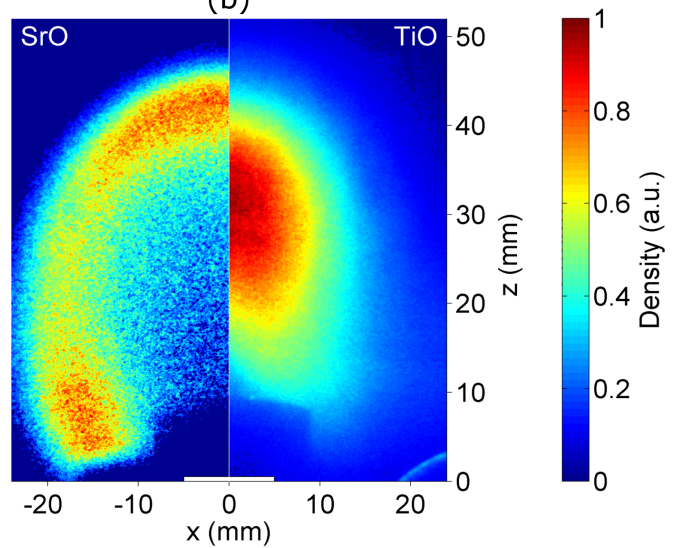

Figure 5.1: Normalized density distributions of several components of an STO plasma plume cross-section in the propagation plane from the target $(z=x=0 \mathrm{~mm})$ to the center of the substrate position ( $x=0 \mathrm{~mm}, z=50 \mathrm{~mm}$ ). The lhs. of (a) displays the $\mathrm{Ti}$ ground state density and the rhs. displays $\mathrm{TiO}$. In (b), respectively, SrO and $\mathrm{TiO}$ are displayed. The densities shown are measured at $20 \mu$ s delay after ablation and obtained with 0.1 mbar pure $\mathrm{O}_{2}$ as background gas. The lack of signal close to the center of the target ( $x=0, z=0)$ is caused by the target holder obscuring one edge of the LIF excitation beam.

$\mathrm{z} \approx 50 \mathrm{~mm}$ ). Two images of half of the plasma plume are shown next to each other for easier comparison. Figure 5.1.a shows the Ti distribution on the left and the TiO distribution on the right as recorded with the 0.1 mbar background consisting of $100 \% \mathrm{O}_{2}$ gas, at $20 \mu$ s delay after ablation, when the front of the plume has not yet reached the substrate region (around $\mathrm{z}=50 \mathrm{~mm}$ ). Figure 5.1.b shows the distribution of SrO (left) next to $\mathrm{TiO}$ (right) under the same conditions. These measurements display a strong spatial separation of the different plasma species. It can be seen that $\mathrm{SrO}$ is located primarily in the outer most edges of the plume, whereas TiO is largely absent from the plasma edges and is concentrated in the center of the plume. Neutral titanium is found to be confined even more to the center, as well as close to the target.

In Fig. 5.2 are shown the ground state population densities of $\mathrm{Ti}$, $\mathrm{TiO}$ and $\mathrm{SrO}$ along the propagation axis (at $\mathrm{x}=0 \mathrm{~mm}$ in Fig. 5.1) as function of the distance from the target, $\mathrm{z}$, at different partial pressures of $\mathrm{O}_{2}$. The densities and oxidation state of plasma species along the propagation axis are of particular interest, especially close to the substrate location, as this displays the material that arrives at the center of the substrate, the location where growth is typically being optimized. Species further away from the propagation axis will either deposit on the outer ranges or edges of the substrate or even disappear into the PLD chamber. These measurements are taken for a delay of $35 \mu$ s between ablation and detection, at which time the plasma plume has expanded to the substrate location, which is typi- 

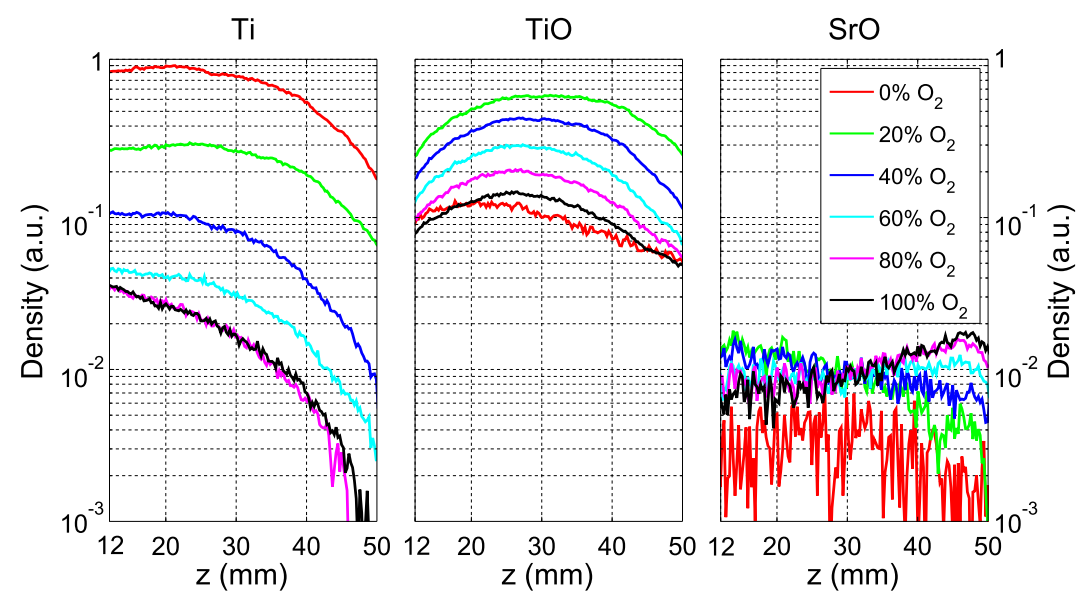

Figure 5.2: The ground state population densities of Ti, TiO and SrO measured along the propagation axis, $z(x=0)$, at different partial pressures of $\mathrm{O}_{2}$. All measurements are done at a delay of $35 \mu$ from target ablation and in 0.1 mbar total background pressure. The first $12 \mathrm{~mm}$ is not shown as it is obstructed by the target holder.

cally located $50 \mathrm{~mm}$ from the target. To emphasize the strong differences in spatial changes of densities, all densities are normalized to the maximum of the Ti, which itself is normalized to unity. Since our approach monitors the relative spatiotemporal distribution for the various species, the ratio in absolute data between different species reflects their ratio in fluorescence yield. The SrO density determined as described above is close to our detection limit $\left(10^{-3}\right.$ in Fig. 5.2), because the LIF signal is only about a factor of ten above the background noise when no $\mathrm{O}_{2}$ is supplied (100\% Ar). The noise is caused by residual spontaneous emission of the plasma that is not removed by the background subtraction. In the Ti distribution, a remarkable drop by almost two orders of magnitude can be seen for all distances from the target when the oxygen fraction is increased but is less than $80 \%$. At $80 \%$ and above (which is pure $\mathrm{O}_{2}$ ) the Ti density does not decrease and the distribution does not change anymore. In other words, the Ti distribution along the z-axis retracts away from the substrate position $(\mathrm{z}=50 \mathrm{~mm})$ with increasing $\mathrm{O}_{2}$ fraction. The on-axis TiO density distribution is low when no $\mathrm{O}_{2}$ is supplied (lowest trace). The highest TiO concentration, at all z-values, is seen with $20 \% \mathrm{O}_{2}$, and decreases moderately with increasing $\mathrm{O}_{2}$ fraction, while the shape of the TiO distribution does not change much except for an overall factor. Another observation is that the density distribution in pure $\mathrm{O}_{2}$ is actually very similar to the case when no $\mathrm{O}_{2}$ is supplied at all (pure Ar). The SrO distribution shows an increase by almost an order of magnitude in density in the front of the expanding plume with increasing $\mathrm{O}_{2}$ fractions, while the density closer to the target slightly decreases. Similar to Ti, the distributions at $80 \%$ and $100 \% \mathrm{O}_{2}$ are nearly identical.

In Fig. 5.3 we show the total amount of $\mathrm{Ti}$, $\mathrm{TiO}$ and $\mathrm{SrO}$ present in the plume 


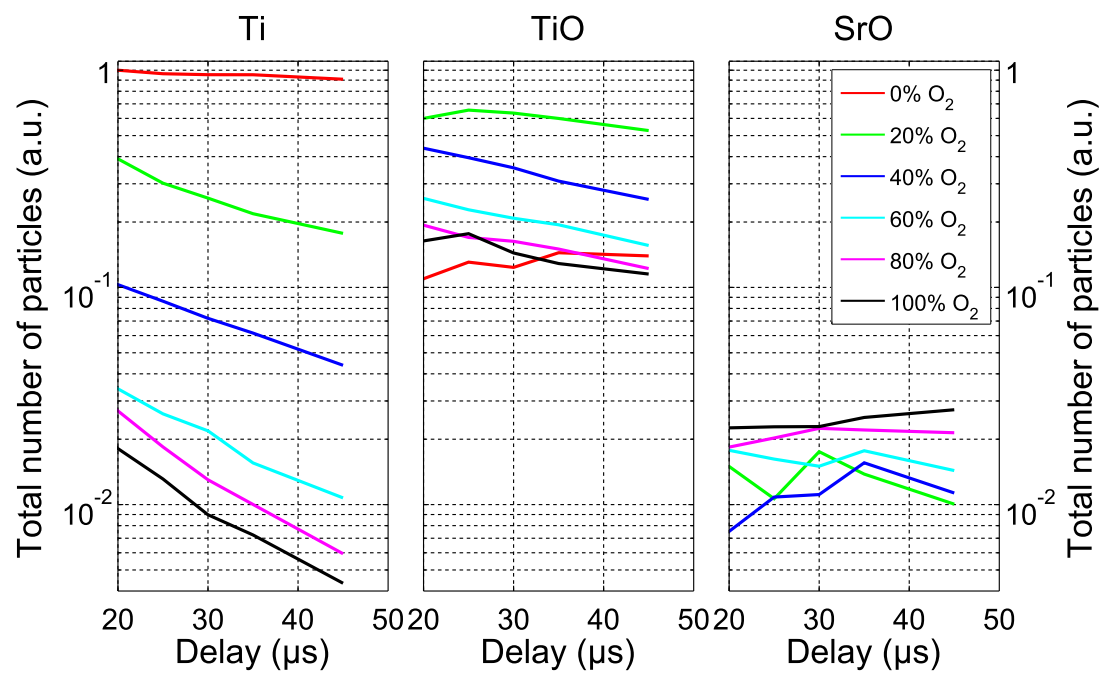

Figure 5.3: Total amount of $\mathrm{Ti}, \mathrm{TiO}$ and $\mathrm{SrO}$ in the plasma plume, acquired by spatially integrating the LIF signal over the entire plume as function of the delay with respect to the moment of target ablation, for different partial pressures of $\mathrm{O}_{2}$. All measurements are at 0.1 mbar total background pressure. In the first $20 \mu \mathrm{s}$, a significant part of the plasma plume is obscured by the target holder and therefore not accessible for LIF measurements.

as function of delay time from target ablation for different partial pressures of $\mathrm{O}_{2}$. This plot allows to identify the chemical processes taking place in the entire plasma plume, and specifically, to separate the influence of the oxygen that is inherently present in the plasma plume, supplied by the target, and the oxygen that is supplied by the background gas. The total number of particles shown in figure 5.3 is acquired by spatially integrating the LIF signal for $\mathrm{Ti}$, $\mathrm{TiO}$ and $\mathrm{SrO}$ over the entire plasma plume, taking the cylindrical symmetry around the $\mathrm{z}$-axis $(\mathrm{x}=\mathrm{y}=0)$ into account. Similar to the density distribution in figure 5.2, all plots of the particle numbers are normalized to the maximum number of particles of $\mathrm{Ti}$, which itself is normalized to unity. The first $20 \mu \mathrm{s}$ is not shown, as part of the plasma plume close to the target is obscured by the target holder and therefore not accessible for LIF measurements. Similar to the density distributions, Ti shows the most prominent change with increasing oxygen fraction. Without any $\mathrm{O}_{2}$ supplied, the total amount of $\mathrm{Ti}$ atoms is nearly constant in time, whereas the Ti amount decreases strongly, by almost two orders of magnitude, when the oxygen fraction is increased. With $\mathrm{O}_{2}$ present, the amount of Ti decreases vs. time with a steepening slope the more $\mathrm{O}_{2}$ is present. In comparison, a large increase in total amount of TiO is visible when a small amount of oxygen is added, but the amount of TiO decreases again with higher $\mathrm{O}_{2}$ fractions, returning to values in pure $\mathrm{O}_{2}$ that are almost identical to those without $\mathrm{O}_{2}$. As function of delay time, the low amount of TiO particles is 
almost constant in a pure Argon background, turning into a weak decrease vs. time with increasing oxygen fraction. The total number of SrO molecules as function of the oxygen fraction in the background gas shows only a small increase. Also, as function of delay time, the number of SrO particles remains almost constant for all oxygen fractions. The strong fluctuations in amount over time are caused by the large amount of noise present close to the detection limit.

Our physical interpretation of the spatiotemporal data in Fig. 5.2 and 5.3, is that a strong mixing of the background gas into the plasma plume takes place, facilitating the oxidation of $\mathrm{Ti}$ to $\mathrm{TiO}$ and of $\mathrm{TiO}$ to $\mathrm{TiO}_{2}$. Both plots show that both the density and amount of titanium steadily decreases with an increasing oxygen fraction in the background gas. Strong mixing of the background gas and plasma plume has been previously concluded by Sasaki et al. [22].

At higher levels of Oxygen ( $80 \%$ and $100 \% \mathrm{O}_{2}$ ) it can be seen that no significant amount of neutral Ti reaches the substrate location. Density distributions at longer delay times even show the front of Ti retracting towards the target. In comparison, the total amount of $\mathrm{Ti}$ in pure Ar remains almost the same over time, indicating that little to no oxidation takes place in the plasma plume with oxygen released from the target. This shows that it is the $\mathrm{O}_{2}$ from the background gas that is responsible for fully oxidizing the plasma species. Interestingly, very little difference is visible between the Ti densities at $80 \%$ and $100 \%$ oxygen, indicating that above a certain threshold, somewhere between $60 \%$ and $80 \%$, the amount of available oxygen no longer is the bottleneck for oxidation of Ti.

The described reaction dynamics are confirmed when inspecting the next species in the oxidation of $\mathrm{Ti}, \mathrm{TiO}$ and $\mathrm{TiO}_{2}$, which is the $\mathrm{TiO}$ concentration. Our measurements show that the density of TiO strongly increases when a small amount of oxygen (20\%) is added to the background gas, but steadily decreases again when the percentage of oxygen is increased further. We conclude that the latter decrease is caused by further oxidation of $\mathrm{TiO}$ to $\mathrm{TiO}_{2}$, because $\mathrm{TiO}$ is an intermediate between $\mathrm{Ti}$ and $\mathrm{TiO}_{2}$ and the Ti density continuously decreases with an increasing $\mathrm{O}_{2}$ fraction, although the $\mathrm{TiO}_{2}$ molecular fluorescence signal turned out to be too weak for detection with our setup.

No SrO is detected in the plasma when ablating in pure argon, or at least only present in small quantities. The density measurement in pure Ar yielded signals of the same strength as the noise, as shown in figure 5.2, making these measurements less reliable. Nevertheless, the lack of $\mathrm{SrO}$ in pure $\mathrm{Ar}$ and the relatively small increase of the amount of $\mathrm{SrO}$ with $\mathrm{O}_{2}$ increasing from $20 \%$ to $100 \%$ suggests that $\mathrm{Sr}$ oxidizes strongly and only requires little $\mathrm{O}_{2}$ to fully oxidize. This is supported by the observation that adding a small amount of oxygen in the background gas, lets $\mathrm{SrO}$ appear in the center of the plasma plume. When increasing the oxygen fraction, an increasing amount of $\mathrm{SrO}$ is formed on the front edge of the plasma plume (figure 5.2), where interaction with the background gas is strongest. A small decrease in SrO amount is visible near the center of the plume. A possible explanation for this behavior is that the reaction $\mathrm{Sr}+\mathrm{O}_{2} \rightleftarrows \mathrm{SrO}+\mathrm{O}$ is strongly dependent on temperature and relative concentration of the four products [23]. As the $\mathrm{O}$ concentration inside the plume will increase due to reactions of $\mathrm{Ti}$ and $\mathrm{TiO}$ 
with $\mathrm{O}_{2}$, the reaction $\mathrm{SrO}+\mathrm{O} \rightarrow \mathrm{Sr}+\mathrm{O}_{2}$ could become more common, leading to a decrease in $\mathrm{SrO}$ concentration in the plume center.

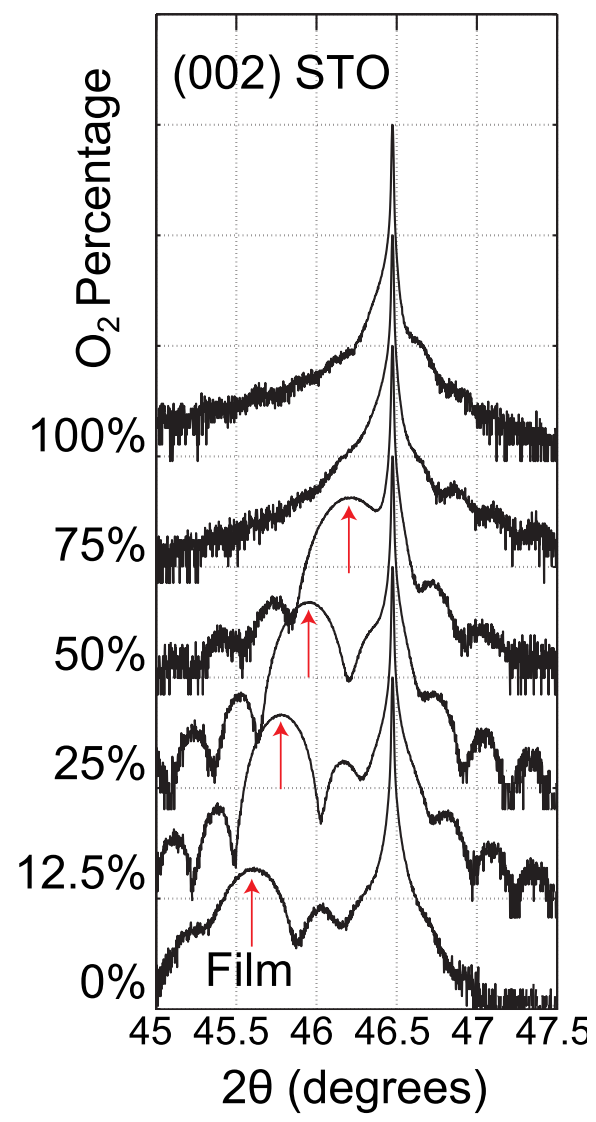

Figure 5.4: XRD scans around the (002) Bragg reflection of STO. It shows scans of samples grown at partial oxygen pressures and a total pressure of $8 \cdot 10^{-2}$ mbar.

The spatiotemporal mapping measurements of $\mathrm{Ti}$, $\mathrm{TiO}$ and $\mathrm{SrO}$ show a significant change in plasma composition of the plume, especially in the front edge of the plume, when it reaches the substrate. To be able to link these plasma composition measurements directly to the thin film growth, we have grown STO films on STO under the same conditions (varying the $\mathrm{Ar} / \mathrm{O}_{2}$ mixture at constant pressure), with the substrate at $\mathrm{z}=50 \mathrm{~mm}$, for a subsequent analysis with X-ray diffraction (XRD). The LIF and XRD measurements are done at slightly different absolute pressures, 0.1 and 0.08 mbar respectively. However, we verified that this small difference does not have a noticeable effect on our results, based on XRD and LIF comparisons of $100 \% \mathrm{O}_{2}$ at 0.08 mbar vs. $80 \% \mathrm{O}_{2}$ at 0.1 mbar. Figure 5.4 shows symmetrical XRD scans around the (002) Bragg reflection of $\mathrm{SrTiO}_{3}$ for these thin films. The film grown in $0 \%$ oxygen (pure Ar) shows a clear film peak, as indi- 
cated by the arrow, aside from the strong substrate peak at 46.5 degrees. This indicates an increased lattice parameter with respect to the substrate[24]. This typically arises from cation defects in the lattice, increasing the volume of the unit cell. For homoepitaxially grown films, the increased volume results in an expansion of the c-axis, since these films are in-plane strained with regard to the $\mathrm{SrTiO}_{3}$ substrate. With increasing $\mathrm{O}_{2}$ fraction, the film peak moves closer to the substrate peak, indicating an improvement in stoichiometry.

A remarkable transition can be seen with further increased oxygen pressure (75\% and above), in that the film peak vanishes. This proves that a significant increase in film quality takes place at a certain oxygen pressure, here at about $75 \%$, where a clear transition takes place from non-stoichiometric to stoichiometric film growth.

At this point we note that a sharp transition to high-quality growth within a narrow range of certain parameter settings is a well-known heuristic observation in PLD. A possible mechanism contributing to the change in stoichiometry might be the effect of preferential scattering, as described by Wicklein et. al. [8]. This effect implies that lighter plasma species scatter stronger than heavier species. In the case of STO, where $\mathrm{Ti}$ is significantly lighter than Sr, the plasma plume would be $\mathrm{Sr}$ rich when it reaches the substrate. However, oxidation of the plasma species increases their mass. Whereas the weight ratio for $\mathrm{Sr} / \mathrm{Ti}$ is 1.8 , for the oxidized species $\left(\mathrm{SrO} / \mathrm{TiO}_{2}\right)$ it is only 1.3 , reducing the effects of preferential scattering. In this paper, we present independent spatiotemporal mapping data of the species in the plasma plume that are responsible for the growth. In fact, when comparing the spectroscopic data with the structural data of the grown material, we find an important correlation: the transition from non-stoichiometric to stoichiometric growth in the XRD measurements coincides with an oxygen pressure where neutral atomic titanium ceases to reach the substrate, while both $\mathrm{Ti}$ and $\mathrm{Sr}$ reach the substrate in their fully oxidized state, which are, respectively, $\mathrm{TiO}_{2}$ and $\mathrm{SrO}$. Furthermore, the decrease of non-stoichiometry aligns with the decrease of $\mathrm{Ti}$ and with the increase of oxidized species. This strongly suggests that full oxidation of plasma constituents is clearly a necessary requirement for stoichiometric growth of STO.

\subsection{Discussion \& Conclusions}

To summarize, we have mapped dynamic oxidation processes of plasma species in the PLD process and have shown the strong influence of the composition of the background gas on the plasma plume composition. Comparing these spectroscopic data with X-ray diffraction data of the grown material quality, we find a clear correlation between the chemical composition of the leading edge of the plasma plume and the stoichiometry of the grown film. Above a specific $\mathrm{O}_{2}$ fraction of the background gas, approximately 75\%, when full oxidation of $\mathrm{Ti}$ and $\mathrm{Sr}$ in the leading edge of the plasma plume is reached, the film growth transides from nonstoichiometric to stoichiometric growth, as determined with X-Ray diffraction. This 
suggests that the oxidation of species of the plasma is a crucial mechanism in the stoichiometric reconstruction of the synthesized oxide thin films.

To illustrate how our findings may be applied in scaling stoichiometric PLD growth, one may consider the following example. If the target-substrate distance is decreased from $50 \mathrm{~mm}$ to $40 \mathrm{~mm}$, while maintaining all other parameter settings, this would prohibit stoichiometric film growth, even in pure $\mathrm{O}_{2}$, as figure 2 shows that the oxidation of Ti would not be complete. Similarly, an increase in targetsubstrate distance would allow for stoichiometric growth in lower oxygen fractions than $75 \%$, as the oxidation is allowed to progress further. Other examples and according spatiotemporal mapping can be devised for transversely upscaling PLD, for increasing the area of stoichiometric growth. 


\section{References}

${ }^{1}$ E. Bousquet, M. Dawber, N. Stucki, C. Lichtensteiger, P. Hermet, S. Gariglio, J. Triscone, and P. Ghosez, "Improper ferroelectricity in perovskite oxide artificial superlattices", Nature 452, 732 (2008).

${ }^{2}$ A. Ohtomo and H. Hwang, "A high-mobility electron gas at the $\mathrm{LaAlO}_{3} / \mathrm{SrTiO}_{3}$ heterointerface", Nature 427, 6973 (2004).

${ }^{3}$ N. Reyren, S. Thiel, A. Caviglia, L. F. Kourkoutis, G. Hammerl, C. Richter, C. Schneider, T. Kopp, A.-S. Rüetschi, D. Jaccard, M. Gabay, D. A. Muller, J.-M. Triscone, and J. Mannhart, "Superconducting interfaces between insulating oxides", Science 317, 1196 (2007).

${ }^{4}$ R. Eason, ed., Pulsed laser deposition of thin films (John Wiley \& Sons, Inc., 2007).

${ }^{5}$ T. Ohnishi, M. Lippmaa, T. Yamamoto, S. Meguro, and H. Koinuma, "Improved stoichiometry and misfit control in perovskite thin film formation at a critical fluence by pulsed laser deposition”, Applied Physics Letters 87, 241919 (2005).

${ }^{6}$ C. Xu, S. Wicklein, A. Sambri, S. Amoruso, M. Moors, and R. Dittmann, "Impact of the interplay between nonstoichiometry and kinetic energy of the plume species on the growth mode of $\mathrm{SrTiO}_{3}$ thin films", Journal of Physics D: Applied Physics 47, 034009 (2014).

${ }^{7}$ S. Amoruso, C. Aruta, P. Aurino, R. Bruzzese, X. Wang, F. M. Granozio, and U. S. di Uccio, "Oxygen background gas influence on pulsed laser deposition process of $\mathrm{LaAlO}_{3}$ and $\mathrm{LaGaO}_{3}$ ", Applied Surface Science 258, 9116 (2012).

${ }^{8}$ S. Wicklein, A. Sambri, S. Amoruso, X. Wang, R. Bruzzese, A. Koehl, and R. Dittmann, "Pulsed laser ablation of complex oxides: the role of congruent ablation and preferential scattering for the film stoichiometry", Applied Physics Letters 101, 131601 (2012).

${ }^{9}$ S. Amoruso, B. Toftmann, and J. Schou, "Thermalization of a uv laser ablation plume in a background gas: from a directed to a diffusionlike flow", Physical Review E: Statistical, Nonlinear, and Soft Matter Physics 69, 056403 (2004).

${ }^{10}$ K. Orsel, R. Groenen, H. Bastiaens, G. Koster, G. Rijnders, and K.-J. Boller, "Spatial and temporal mapping of al and alo during oxidation in pulsed laser ablation of $\mathrm{LaAlO}_{3}$ ", Journal of Instrumentation 8, C10021 (2013).

${ }^{11}$ C. Aruta, S. Amoruso, R. Bruzzese, X. Wang, D. Maccariello, F. M. Granozio, and U. S. di Uccio, "Pulsed laser deposition of $\mathrm{SrTiO}_{3} / \mathrm{LaGaO}_{3}$ and $\mathrm{SrTiO}_{3} / \mathrm{LaAlO}_{3}$ : plasma plume effects", Applied Physics Letters 97, 252105 (2010).

${ }^{12}$ A. Sambri, D. Cristensen, F. Trier, Y. Chen, S. Amoruso, N. Pryds, R. Bruzzese, and $\mathrm{X}$. Wang, "Plasma plume effects on the conductivity of amorphous- $\mathrm{LaAlO}_{3} / \mathrm{SrTiO}_{3}$ interfaces grown by pulsed laser deposition in $\mathrm{O}_{2}$ and Ar", Applied Physics Letters 100, 231605 (2012).

${ }^{13}$ H. Döbele, T. Mosbach, K. Niemi, and V. S.-v. der Gathen, "Laser-induced fluorescence measurements of absolute atomic densities: concepts and limitations", Plasma Sources Science and Technology 14, S31 (2005). 
${ }^{14}$ T. Okada and M. Maeda, "Laser spectroscopic studies of pulsed-laser deposition process for high- $\mathrm{T}_{c}$ thin films", Materials Science \& Engineering, B: Solid-State Materials for Advanced Technology 47, 64 (1997).

${ }^{15}$ C. Dutouquet and J. Hermann, "Laser-induced fluorescence probing during pulsed-laser ablation for three-dimensional number density mapping of plasma species", Journal of Physics D: Applied Physics 34, 3356-3363 (2001).

${ }^{16}$ Y. Nakata, T. Okada, M. Maeda, S. Higuchib, and K. Ueda, "Effect of oxidation dynamics on the film characteristics of Ce:YIG thin films deposited by pulsedlaser deposition", Optics and Lasers in Engineering 44, 147 (2006).

${ }^{17}$ A. Kramida, Y. Ralchenko, J. Reader, and N. A. T. (2013), Nist atomic spectra database (ver. 5.1), National Institute of Standards and Technology, (2013) http: //physics.nist.gov/asd.

${ }^{18} \mathrm{R}$. Pearse and A. Gaydon, The identification of molecular spectra, 3rd ed. (Chapman and Hall, London, 1976).

${ }^{19}$ A. Sambri, S. Amoruso, X. Wang, M. Radovic', F. M. Granozio, and R. Bruzzese, "Substrate heating influence on plume propagation during pulsed laser deposition of complex oxides", Applied Physics Letters 91, 151501 (2007).

${ }^{20} \mathrm{R}$. Groenen, "Understanding thin film growth in pulsed laser deposition; plasma plume chemistry".

${ }^{21}$ X. Zhuang, A. Le, T. C. Steimle, R. Nagarajan, V. Gupta, and J. P. Maier, "Visible spectrum of titanium dioxide", Physical Chemistry Chemical Physics 12, 15018 (2010).

${ }^{22}$ K. Sasaki and H. Watarai, "Dynamics of laser-ablation plume and ambient gas visualized by laser-induced fluorescence imaging spectroscopy", Japanese Journal of Applied Physics 45, L447 (2006).

${ }^{23}$ C. Batalli-Cosmovici and K.-W. Michel, "Molecular beam study on bao and sro formation for clarifying interaction of meta-vapors with upper atmosphere oxygen", Planetary and Space Science 21, 89 (1972).

${ }^{24}$ B. Jalan, P. Moetakef, and S. Stemmer, "Molecular beam epitaxy of $\mathrm{SrTiO}_{3}$ with a growth window", Applied Physics Letters 95, 032906 (2009). 


\section{LASER-INDUCED FLUORESCENCE ANALYSIS OF PLASMAS FOR STOICHIOMETRIC GROWTH OF $\mathrm{YBIO}_{3}$ FILMS WITH PULSED LASER DEPOSITION}

By applying two-dimensional laser-induced fluorescence (LIF) on multiple plasma constituents, we are able to directly link, for the first time in a $\mathrm{YBiO}_{3}(\mathrm{YBO})$ plasma, the influence of oxygen present in the background gas during pulsed laser deposition (PLD) on the oxidation of plasma species as well as the formation of stoichiometric perovskite films. With spatiotemporal LIF mapping of the plasma species in different background gas compositions, we find that little direct chemical interaction appears to take place between the plasma plume constituents and the background gas. However, a strong influence of the background gas composition can be seen on the YBO film growth, as well as a strong correlation between the oxygen fraction in the background gas and the amount of YO in the plasma plume. We believe this is caused not by a mixing and reaction of the plasma plume with the background gas, but by interaction between the background gas and the target as well as the substrate.

\section{Introduction}

It is widely accepted that pulsed laser deposition (PLD) allows for stoichiometric transfer of complex materials, making it a very powerful and universal deposition technique for the growth of thin films of complex crystalline oxides such as to impose ferro-electricity [1], two-dimensional electron gases at heterointerfaces [2] or superconductivity at interfaces [3], among other applications [4]. Recently, a 
new class of materials, so-called topological insulators (TIs), have attracted much of attention $[5,6]$ due to their unique quantum-mechanical properties, that provide time-reversal-symmetry-protected surface states [7]. Many different TI materials have already been discovered [6], specifically materials based on Bismuth such as $\mathrm{BiTe}_{3}[8]$ and $\mathrm{BiSe}_{3}[9,10]$.

Of particular promise, based on first-principles calculations [11] appears to be $\mathrm{YBiO}_{3}$. Although YBO thin films have been grown by chemical solution deposition $[12,13]$, so far very few attempts to grow single-crystalline films with PLD has been made. A first difficulty for growing YBO via PLD may be related to an unfavorable ratio of the ionic radii of the cations, which can prevent the growth of bulk and single crystalline films. A quantitative rule for the latter is known as Goldschmidt's tolerance factor, which depends on the ratio of the radii of the two cations. For growth of a stable perovskite the factor has to be larger than 0.71 [14], while for YBO, this factor is slightly smaller, 0.669 , indicating that the perovskite crystal structure might easily become distorted during growth.

A second problem with stoichiometric growth of YBO in PLD is found in the volatility of $\mathrm{Bi}$, and that $\mathrm{Bi}$ hardly oxidizes. These properties result in a low sticking factor, causing little Bi to be available for synthesizing a stoichiometric YBO film.

The actual degree of influence of these factors on the much reduced reliability and predictability of appropriate growth parameters for PLD yielding stoichiometric YBO is unknown. A question is, e.g., to what degree $\mathrm{Bi}$ is oxidized by the $\mathrm{O}_{2}$ background gas before deposition onto the substrate or whether the oxygen background rather leads to oxidation only at the substrate, after an atomic deposition of $\mathrm{Bi}$ and Y. In our previous work involving $\mathrm{LaAlO}_{3}[15]$ and $\mathrm{SrTiO}_{3}$ [15] we have found that the plasma dynamics and chemistry and, particularly, where and when in the plume oxidation occurs, is of decisive influence on the morphology of the grown films.

Using laser-induced fluorescence spectroscopy, spatially and temporally resolved distributions of constituents of the plasma can be obtained that allow to trace the underlying dynamical and chemical processes, such as the propagation and oxidation of the most relevant plasma species. The research in this paper focuses on the propagation and oxidation of the constituents in an YBO plasma. We record the spatiotemporal distributions of $\mathrm{Y}, \mathrm{Bi}$ and $\mathrm{YO}$ while systematically varying the chemical composition of the background gas. The latter is achieved via changing the relative concentrations of $\mathrm{O}_{2}$ and $\mathrm{Ar}$, while maintaining the absolute total pressure constant. The composition of the plasma plume is then linked to the composition, stoichiometry and morphology of the film growth, using sensitive X-ray diffraction (XRD) measurements.

We find that little direct chemical interaction appears to take place between the plasma plume constituents and the background gas. However, a strong influence of the background gas composition can be seen on the YBO film growth, as well as a strong correlation between the oxygen fraction in the background gas and the amount of YO in the plasma plume. We believe this is caused not by a mixing and reaction of the plasma plume with the background gas, but by interaction between the background gas and the target as well as the substrate. 


\subsection{Experimental setup and methodology}

The spatiotemporal mapping of the plasma constituents is carried out in a custom built PLD chamber, a detailed description of which can be found in previous articles of ours $[15,16]$ and in chapter 3 . The target ablation is carried out with laser pulses generated by a $\mathrm{KrF}$ excimer laser ( $248 \mathrm{~nm}, 30 \mathrm{~ns}$ duration FWHM, operating at $2 \mathrm{~Hz}$ ). A mask with a rectangular hole, placed in the $\mathrm{KrF}$ laser beam to select a spatially uniform beam, is imaged onto the target, resulting in a laser spot of $0.91 \times 2.42 \mathrm{~mm}^{2}$ in size. Through control of the laser output energy, the laser fluence at the target is kept constant at $1.3 \pm 10 \% \mathrm{~J} / \mathrm{cm}^{2}$. A sintered target consisting of $99.99 \%$-purity $\mathrm{Y}_{2} \mathrm{O}_{3}$ and $\mathrm{Bi}_{2} \mathrm{O}_{3}$ in a one-to-one molar ratio was used during all measurements.

A fixed value for the total background pressure of $0.1 \mathrm{mbar}$ is chosen. At this pressure the plasma expansion is no longer in the ballistic regime but undergoes a strong interaction with the background gas, moderating the kinetic energy of the ablated species [17]. The choice of a mixture of argon and oxygen as background gas is motivated by their similar atomic weight. Thereby the PLD plasmas exhibit very similar expansion dynamics, propagation speed and collision rates, for all gas mixtures, ruled by the total pressure and rather independent of the partial pressures of $\mathrm{Ar}$ and $\mathrm{O}_{2}$ [18]. As the ablation laser fluence is kept constant for all measurements, changes in plasma composition will be primarily caused by a change in chemical processes and not by any changes in dynamics.

We use laser-induced fluorescence (LIF), which enables us to excite and detect ground state species at freely selectable locations and times, and in a chemically specific manner. The UV excitation wavelengths we use for generating LIF in the YBO plasma range from 250 to $350 \mathrm{~nm}$ and are generated by frequency doubling the output of a dye laser, pumped with the second harmonic (532 nm) output of a Q-switched Nd:YAG laser (7 ns FWHM). The UV output has a pulse duration of $4 \mathrm{~ns}$ FWHM, a bandwidth of $1.6 \mathrm{pm}$ and an energy of $80 \mu \mathrm{J}$ per pulse. Using cylindrical telescope optics the LIF excitation beam is transformed into a thin sheet. It illuminates a cross-section of the plasma plume in the plane of the forward propagation from the ablation spot on the target to the center of the deposition substrate. The sheet has an in-plane focus of approximately $0.4 \mathrm{~mm}$ thickness.

To image the neutral yttrium distribution in the plasma plume, we use a transition from the atomic ground state $\left(d 5 s^{2} a^{2} D\right)$ to the $d 25 p x^{2} D$ state at 294.8403 $\mathrm{nm}$. Relaxation occurs to the $d 25 s a^{2} F, d 25 s a^{4} P$ and $d 25 s b^{2} D$ states via fluorescence at several lines between $535 \mathrm{~nm}$ and $565 \mathrm{~nm}$ [19]. A colored glass filter with a bandpass of 525 to $575 \mathrm{~nm}$ transmits the LIF of Y while suppressing most of the thermally induced spontaneous emission (SE) and blackbody radiation of the plasma.

The excitation of yttrium oxide is achieved using a $A^{2} \Pi \rightarrow X^{3} \Sigma$ transition at $597.22 \mathrm{~nm}$ [20], following detection of red-shifted fluorescence (around $600 \mathrm{~nm}$ ) through a 575 to 625 transmission filter.

The excitation of atomic bismuth is done at the transition from the ground state $\left(6 p^{3}{ }^{4} S^{\circ}\right)$ to the $6 p^{2}\left({ }^{3} P_{0}\right) 7 s$ excited state at a wavelength of $306.7700 \mathrm{~nm}$ [21]. 
Relaxation to the $6 p^{3}{ }^{2} D^{\circ}$ state via emitting fluorescence at $472.25 \mathrm{~nm}$ is used for detection. A dielectric filter with a bandpass of 465 to $485 \mathrm{~nm}$ transmits the LIF. To remove residual spontaneous emission from the plasma that is transmitted by the band-pass filters, a background subtraction using measurements with the LIF beam blocked is applied to all LIF measurements. The influence of shot-toshot fluctuations is reduced by averaging the recorded fluorescence images over 30 shots. Averaging over more than 30 shots was found to not significantly improve the measurements.

To improve the sensitivity and accuracy of the spatiotemporal mapping, we decided to carry out the measurements with the substrate removed, to avoid unwanted reflections at the substrate of the LIF beam that traverses the plasma plume at an oblique angle with respect to the axis between target and substrate. This modification prevents artifacts in the spatial distribution mapping for the relevant observation times, when the plume arrives at the location of the substrate.

The films deposited for the XRD measurements are grown on a substrate heated to $670^{\circ} \mathrm{C}$. The influence of substrate heating on the propagation dynamics of the plasma plume [22], present during deposition but absent during LIF measurements, is largely avoided by using laser substrate heating instead of the more commonly used resistive substrate heating. We performed comparative measurements of plume propagation with either a laser heated target at $710^{\circ} \mathrm{C}$ or at room temperature [23], which showed little difference, demonstrating the validity of the LIF measurements without heated substrate. In contrast, plume propagation with a resistively heated substrate at $710^{\circ} \mathrm{C}$ showed, compared to room temperature, large changes in propagation similar as described by Sambri et al. [22].

To be able to relate the LIF signal to the material density, all excitation transitions used are driven into saturation. In saturation, a variation in laser power, for example, pulse-to-pulse fluctuations, typically around 10\%, or spatial inhomogeneities, will result in only small variations in the LIF signal $(<2 \%$, see section 3.4). Thereby, the LIF signal becomes close to proportional to the density of the excited species. However, a calibration factor is required to convert the relative density maps recorded by the iCCD camera into maps that display absolute values of the particle density.

As the method to provide this calibration factor we have chosen absorption spectroscopy (AS), similar to the method described by Dutouquet et al. [24]. AS can be used to measure the spatially integrated density of particles along the LIF excitation beam path, i.e., it does not provide any information on the spatial shape of the density distribution. However, as is described in section 2.3, the spatially integrated number of particles excited by the LIF beam is proportional to the spatially integrated LIF signal, thus providing an absolute calibration of the density distribution. In section 3.5 we show how AS is implemented into the experimental setup.

In our experiments the absolute calibration of LIF with AS was only applicable to the atomic species and not to molecules for several reasons. Firstly, molecules typically have an absorption cross-section that is several orders of magnitude lower than the absorption cross-section of atoms, which rendered the absorption to be 
lower than our detection limit. Secondly, for the molecules investigated here, the absorption cross-sections are not well known, such that it would be difficult to translate absorption signals into molecular densities. Lastly, for atoms it can be assumed that the vast majority is in the ground state, as the plasma temperature (approximately $5.000 \mathrm{~K}$ after $<10 \mu \mathrm{s}$ ) is too low to thermally excite electronic transitions in the atoms. However, molecules typically have transitions of much lower excitation energies in the form of rotational and vibrational transitions. Therefore, a significant fraction is expected to be in an excited state due to thermal excitation. Because the temperature distribution of the plasma plume is not known, it is not possible, without additional information, to correct for the ratio between the ground-state and excited-state populations to obtain a measurement of the absolute density.

\subsection{Results}

To investigate the chemical composition of the plasma plume and, more specifically, the effects of the background gas composition on the oxidation of the plasma constituents, we aimed to map the spatiotemporal distribution of all relevant and expectantly dominant plasma constituents which are Y, YO, Bi and BiO. From the distribution we can determine plasma composition when it reaches the substrate position. All species are mapped in a background gas of which the total pressure is held constant at 0.1 mbar. The partial pressure of $\mathrm{O}_{2}$ is step-wise increased from $0 \%$ to $100 \%$ in order to step-wise increase the oxidation of $\mathrm{Bi}$ and Y in the plasma plume. Measuring BiO densities, however, proved impossible using the excitation lines at $338.10 \mathrm{~nm}, 343.44 \mathrm{~nm}, 670.88 \mathrm{~nm}$ and $693.65 \mathrm{~nm}$ described by Pearse and Gaydon [20]. As several of these transitions are described as strong resonance lines, we suspect that the concentrations of $\mathrm{BiO}$ are below the detection limit of our setup. Unfortunately, as the detection limit of our setup is dependent on several species-specific factors, such as the strength of the transition being used and the wavelength being detected, no upper limit can be given for the BiO density.

Figure 6.1 shows typical results of distribution measurements for a crosssection of the plasma plume in the propagation plane from the target $(\mathrm{z}=\mathrm{x}=$ $0 \mathrm{~mm}$ ) to where the substrate is positioned during growth experiments ( $\mathrm{x}=0 \mathrm{~mm}$, $\mathrm{z} \approx 50 \mathrm{~mm}$ ). Two images of half of the plasma plume are shown next to each other for easy comparison. The lhs. of each image displays the ground state density distribution as recorded with a 0.1 mbar background consisting of $100 \% \mathrm{O}_{2}$, whereas the rhs. displays the distribution recorded with a pure Ar background gas. All measurements shown in Fig. 6.1 are carried out at $35 \mu$ s delay after ablation, when the front of the plume starts reaching the region of the substrate (around $\mathrm{z}=50 \mathrm{~mm}$ ). All images are normalized to unity, with the normalization factor displayed in the bottom, which corresponds to the highest density present in each picture. Figure 6.1(a) shows the Bi distribution for YBO ablation in pure $\mathrm{O}_{2}$ and Ar, side by side. Although visually appearing quite similar in spatial shape, a factor of 2.5 difference in absolute density is present between the two cases. It should 
(a) Bi LIF

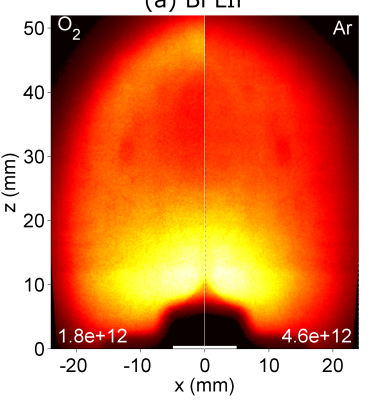

(b) Y LIF

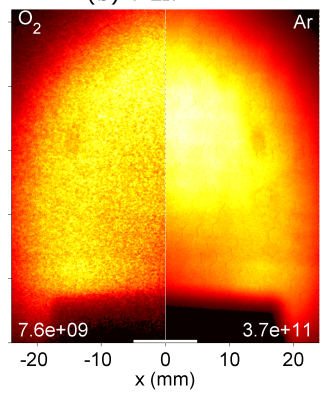

(c) YO LIF

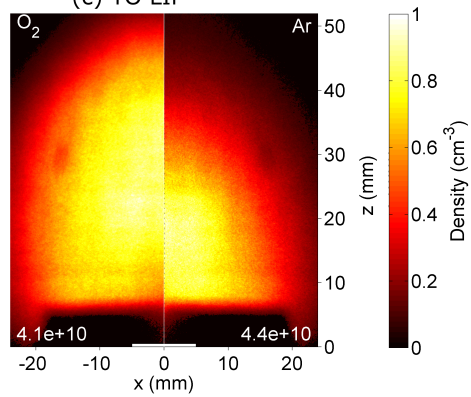

Figure 6.1: Density distributions of several components of an YBO plasma plume cross-section in the propagation plane from the target $(z=x=0 \mathrm{~mm})$ to the center of the substrate position $(x=0 \mathrm{~mm}, z=50 \mathrm{~mm}$ ). The lhs. of each picture displays the ground state density in 0.1 mbar of pure $\mathrm{O}_{2}$ background gas and the rhs. displays the density in a 0.1 mbar pure Ar background. All pictures are normalized, with the normalization factor shown in the bottom. The densities shown are measured at 35 $\mu$ s delay after ablation. The lack of signal close to the center of the target ( $x=0, z=$ 0 ) is caused by the target holder obscuring one edge of the LIF excitation beam.

be noted that a significant amount of Bi appears to remain close to the ablation target, while all other species we have studied propagate towards the substrate, similar to $\mathrm{Y}$ in this figure. The distribution of $\mathrm{Y}$, as shown in Fig. 6.1(b), displays little difference in relative spatial distribution again, but does show a very large difference in absolute density by almost two orders of magnitude.

The largest difference in the spatial shape of the distribution for a species formed after ablation in either pure $\mathrm{O}_{2}$ or Ar is visible in YO (Fig. 6.1(c)). Although the maximum absolute density changes only slightly for the two different background gas compositions, in pure $\mathrm{O}_{2}$ the $\mathrm{YO}$ distribution has propagated significantly more towards the substrate location $(z=50 \mathrm{~mm})$ as compared to the distribution in pure Ar.

To reveal more details, Fig. 6.2(a) shows the ground state population densities of $\mathrm{Y}, \mathrm{YO}$, and $\mathrm{Bi}$ along the propagation axis, $\mathrm{z}$ (at $\mathrm{x}=0 \mathrm{~mm}$ in Fig. 6.1), at $50 \mathrm{~mm}$ from the target, as a function of different partial pressures of $\mathrm{O}_{2}$ in the background gas mixture. The measurements are taken at $35 \mu$ s delay from target ablation. It can be seen that the density of $\mathrm{Y}$ decreases by almost two orders of magnitude with an increase of $\mathrm{O}_{2}$ up to $60 \%$, while the density remains stable with further increasing the $\mathrm{O}_{2}$ concentration. The density of $\mathrm{YO}$ only increases significantly from $0 \% \mathrm{O}_{2}$ to $20 \% \mathrm{O}_{2}$, after which an increase in oxygen fraction has very little influence on the $\mathrm{YO}$ density. In contrast, the Bi density is almost independent of the composition of the background gas and shows only a marginal decrease for increasing $\mathrm{O}_{2}$ fractions.

In Fig. 6.2(b) we show the total (i.e., spatially integrated) amount of Y, YO, and $\mathrm{Bi}$ present in the plume as function of partial pressures of $\mathrm{O}_{2}$ as measured at $35 \mu$ s delay from target ablation. This plot allows to identify the chemical pro- 

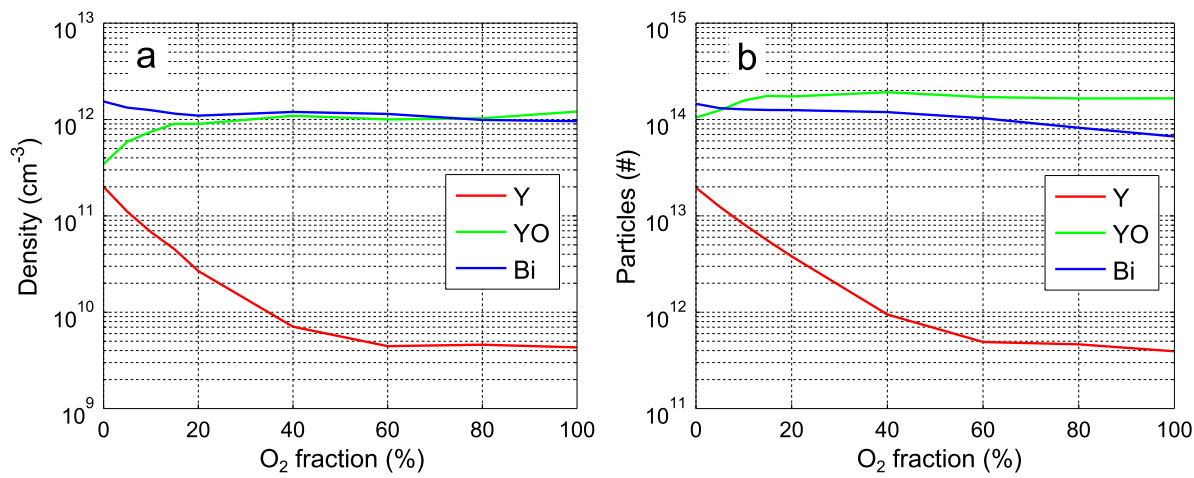

Figure 6.2: (a) The ground state population densities of $Y, Y O$ and Bi measured along the propagation axis, $z(x=0)$, at $50 \mathrm{~mm}$ from the target, at different partial pressures of $\mathrm{O}_{2}$. (b) Total number of ground state particles of $Y$, YO and Bi present in the plasma plume at different partial pressures of $\mathrm{O}_{2}$. All measurements are done at a delay of $35 \mu$ from target ablation and in 0.1 mbar total background pressure.

cesses taking place anywhere in the entire plasma plume, and specifically, it allows to separate the influence of oxygen that is inherently present in the plasma plume (supplied by the $\mathrm{Y}_{2} \mathrm{O}_{3} / \mathrm{Bi}_{2} \mathrm{O}_{3}$-target), and the oxygen that is supplied via the background gas.

The total number of particles shown in Fig. 6.2(b), determined by spatially integrating the LIF signal for $\mathrm{Y}$ and $\mathrm{Bi}$ over the entire plasma plume, assumes a cylindrical symmetry around the $\mathrm{z}$-axis $(\mathrm{x}=\mathrm{y}=0)$. To calculate the amount of YO present in the plume, we assume that the primary oxidation product for $\mathrm{Y}$ is YO. This assumption is supported by the fact that the total number of Y-containing particles, i.e., $\mathrm{N}_{Y}+\mathrm{N}_{Y O}$, remains nearly constant for all oxygen fractions.

To determine the total number of Y-containing particles present in the YBO plasma plume with an independent approach, we determined the total amount of ablated material using a measurement of weight reduction of the target. This measurement yielded a number of around $1 \times 10^{15}$ particles per pulse. Since one fifth of the particles in a plasma produced by ablating $\mathrm{YBiO}_{3}$ is $\mathrm{Y}, \mathrm{N}_{Y}+\mathrm{N}_{Y O}=$ $2 \cdot 10^{14}$ per pulse.

Comparing this number with Fig. 6.2(b) shows that even in pure Ar, a significant amount of $\mathrm{Y}$ is oxidized $(>50 \%)$. When more oxygen is supplied via the background gas, the amount of $Y$ further decreases until the oxidation appears to reach a chemical equilibrium between loss to $\mathrm{YO}$ and the reverse reaction for an oxygen fraction of $60 \%$ and above. The amount of YO increases slightly when going from pure Ar to $20 \% \mathrm{O}_{2}$, beyond which it remains the same for higher oxygen fractions. The amount of $\mathrm{Bi}$ displays a steady decrease with an increase in $\mathrm{O}_{2}$ to half the amount of $\mathrm{Bi}$ in pure oxygen as compared to pure Ar. As oxidation of half of the amount of $\mathrm{Bi}$ to $\mathrm{BiO}$ would lead to a $\mathrm{BiO}$ density that we believe should fall well within the detection limits of our setup, we do not believe this to be the 
primary channel for loss of Bi.

Our physical interpretation of the spatiotemporal data in Fig. 6.1 and 6.2, is that only limited chemical interaction takes place between the plasma plume and the background gas. Thereby the measurements in the YBO plasma show remarkable differences in comparison to oxidation studies done on LAO [16] and STO [15].

To better describe the difference to those observations, in the oxidation studies of $\mathrm{Ti}$ and $\mathrm{Al}$, we recall three distinct features that can be found as characteristic for oxidation of a plasma species by $\mathrm{O}_{2}$ from the background gas. Firstly, oxidation should be largely absent from the plasma for ablation in pure Ar, only appearing when oxygen is added to the background gas. Secondly, when increasing the oxygen fraction, species should become oxidized primarily on the outer edges of the plume, since in this region the interaction between the plasma plume and the background gas is strongest. Only in a background gas close to pure oxygen can oxidized species also appear in the center of the plasma plume. And thirdly, a loss of the atomic species on the edges of the plasma plume should be accompanied by the appearance of the oxidized species in these regions, following a chemical reaction converting the atomic species (e.g., A) to the oxidized species (A $+\mathrm{O}_{2} \rightarrow$ $\mathrm{AO}+\mathrm{O})$.

None of these features typical for oxidation by reactions with the background gas appear to be present in the oxidation of Y. From Fig. 6.1 it is evident that large amounts of YO are present in the plasma plume when ablating in pure Ar, while the oxidized species appear to emanate from the center of the plume, and the $Y$ density decreases homogeneously, instead of primarily at the edges.

These differences lead us to conclude that, in an YBO plasma, the atomic O provided to the plasma from the target during ablation is the primary source of oxygen for the oxidation of $\mathrm{Y}$. The increase of $\mathrm{YO}$ with an increase of the $\mathrm{O}_{2}$ background fraction might be caused by oxidation of the target surface in-between ablation pulses. As a supporting argument for this suggestion, it has previously been shown that repeated ablation can lead to a change in target composition on the surface [25]. Here, in the case of an YBO target, repeated ablation in pure Ar might have led to partial depletion of oxygen at the surface of the target. However, when ablating the target in a background with $\mathrm{O}_{2}$ present, the surface of the target can oxidize in-between ablation pulses, thereby replenishing the oxygen content of the target. Bismuth appears to hardly oxidize at all, which is consistent with the observation that the reaction between $\mathrm{Bi}$ and $\mathrm{O}_{2}$ is endothermic [26].

To be able to link the changes in plasma composition directly to the thin film growth, we have grown YBO films on Lanthanum Strontium Aluminium Tantalum Oxide (LSAT) under the same conditions, again varying the $\mathrm{Ar} / \mathrm{O}_{2}$ mixture at constant pressure, with the substrate placed at $\mathrm{z}=50 \mathrm{~mm}$, for a subsequent analysis with X-ray diffraction (XRD).

Figure 6.3 shows symmetrical XRD scans of YBO films deposited with a total of 900 ablation pulses on LSAT. The strong peaks visible at all oxygen fractions at $23.0^{\circ}$ and $46.9^{\circ}$ correspond to the (001) and (002) Bragg reflections of the LSAT substrate, respectively. A weak Bragg reflection can be seen at $29.0^{\circ}$ for deposition 


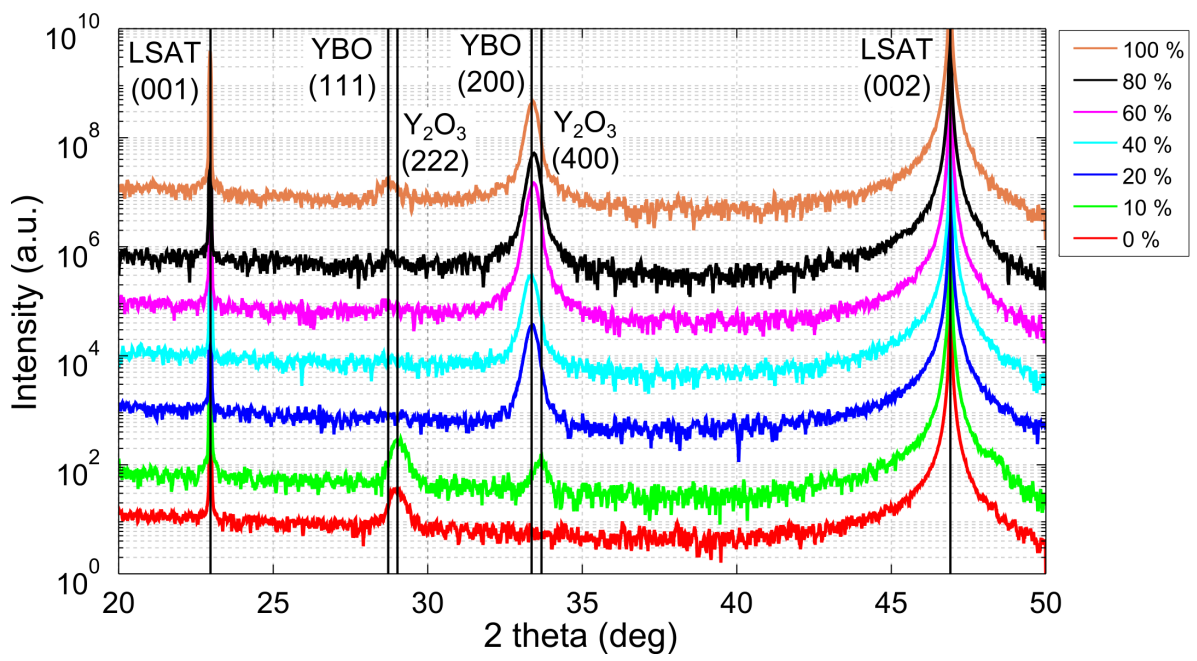

Figure 6.3: XRD scans of YBO grown on LSAT. The vertical black lines indicate several Bragg reflections of the substrate and the thin film. These XRD scans are carried out on samples grown with a total of 900 ablation pulses at different partial oxygen pressures while maintaining a total pressure of $1 \cdot 10^{-1}$ mbar. At oxygen fractions below 20\%, only peaks corresponding to poly-crystalline $\mathrm{Y}_{2} \mathrm{O}_{3}$ are visible. For fractions of 20\% $\mathrm{O}_{2}$ and higher, an epitaxial YBO layer of approximately $35 \mathrm{~nm}$ thick is grown, as indicated by the (200) peak. At oxygen fractions higher than 60\%, a small amount of YBO appears to grow in a different, non-epitaxial orientation, corresponding to the (111) peak (Bragg reflection at $28.7^{\circ}$ ), indicating an increase in nucleation rate of $Y B O$ at the substrate surface.

in pure $\mathrm{Ar}$ and $10 \% \mathrm{O}_{2}$. Additionally, at an oxygen fraction of $10 \%$, a peak at $33.7^{\circ}$ appears. These two peaks correspond to poly-crystalline $\mathrm{Y}_{2} \mathrm{O}_{3}$, at (222) and (400), respectively [27]. The peak at $33.4^{\circ}$, visible for all oxygen fractions of $20 \%$ and above, corresponds to epitaxially grown YBO ((200) Bragg reflection). At $80 \%$ and $100 \% \mathrm{O}_{2}$, a weak YBO peak at $28.7^{\circ}$ is present, which indicates the presence of a small amount of non-epitaxial YBO in the (111) orientation [28]. Using atomic force microscopy, all YBO films were determined to be approximately $35 \mathrm{~nm}$ thick.

At low oxygen fractions ( $0 \%$ and $10 \%$ ), only small amounts of $\mathrm{Y}_{2} \mathrm{O}_{3}$ form on the surface of the substrate. When increasing the $\mathrm{O}_{2}$ to $20 \%$, an epitaxial layer of YBO suddenly appears. For higher oxygen fractions, additional growth of YBO in a non-epitaxial orientation appears, as indicated by the (111) peak at $28.7^{\circ}$. We believe this to be caused by an increase in nucleation rate of YBO with increasing availability of $\mathrm{O}_{2}$ on the substrate surface.

The remarkable transition from the absence of $\mathrm{YBO}$ growth in pure Ar to the growth of a full epitaxial layer in $20 \% \mathrm{O}_{2}$ can be partially explained by the increase in available $\mathrm{YO}$ at the substrate position, as shown in figure 6.2(a). However, already at $10 \% \mathrm{O}_{2}$ a significant amount of $\mathrm{YO}$ is available, yet no YBO appears to 
grow. In previous research [15] we found that the oxidation state in which the plasma constituents arrive at the substrate can influence the stoichiometry of the film growth, yet, film growth would still take place. Other research where the background gas is modified, either its pressure of its composition, to investigate its influence on the film growth $[29,30]$ shows similar results. That is, a change in stoichiometry is present for certain background gas compositions and pressures, but film growth is still present. We therefore suspect that, here, an additional mechanism is responsible for the sudden appearance of an YBO film at $20 \%$ oxygen. Since $\mathrm{Bi}$ appears to arrive largely in its atomic, non-oxidized form, an additional source of oxygen is necessary to form $\mathrm{YBiO}_{3}$ from $\mathrm{YO}$ and $\mathrm{Bi}$. We believe that the oxygen from the background gas, after the arrival of $\mathrm{Bi}$ on the substrate, is an essential component for the synthesis of YBO on the substrate surface.

\subsection{Discussion \& Conclusions}

We have mapped the dynamic oxidation and propagation processes of $\mathrm{Y}$ and $\mathrm{Bi}$ in the plasma plume during PLD of YBO. From the optically recorded Y, YO and Bi spatiotemporal distributions in the plasma, we find that little direct chemical interaction appears to take place between the plasma plume constituents and the background gas. However, a strong influence of the background gas composition can be seen on the plasma composition, which appears not to be caused by a mixing and reaction of the plasma plume with the background gas, but by reactions between the background gas and the target.

Via comparison of the spatiotemporal distributions in the plasma with X-ray diffraction data of the grown material quality, we find a strong increase in nucleation rate of $\mathrm{YBO}$ on the substrate surface with an increase in oxygen fraction in the background gas. Below $20 \% \mathrm{O}_{2}$, no YBO is deposited, only poly-crystalline $\mathrm{Y}_{2} \mathrm{O}_{3}$. At oxygen fractions of $20 \%$ and above, the nucleation rate of $\mathrm{YBO}$ is high enough for a stoichiometric film to grown on the substrate. Around $80 \% \mathrm{O}_{2}$, the nucleation rate has increase enough for a non-epitaxial orientation of YBO to appear, suggesting that the oxygen fraction can be used to fine-tune the film growth.

Remarkably, these transitions in film growth do not coincide with a significant change in plasma composition, as we find that the plasma composition only slightly changes with background gas composition. This has led us to conclude that the strong influence of the background gas composition seen in the XRD measurements is on the actual synthesis of YBO film from the plasma constituents, not on the plasma plume composition. $\mathrm{O}_{2}$ from the background gas appears to be a crucial building block for forming, at the surface of the substrate, $\mathrm{YBiO}_{3}$ from the $\mathrm{Bi}$ and YO provided by the plasma plume. 


\section{References}

${ }^{1}$ E. Bousquet, M. Dawber, N. Stucki, C. Lichtensteiger, P. Hermet, S. Gariglio, J. Triscone, and P. Ghosez, "Improper ferroelectricity in perovskite oxide artificial superlattices", Nature 452, 732 (2008).

${ }^{2}$ A. Ohtomo and H. Hwang, "A high-mobility electron gas at the $\mathrm{LaAlO}_{3} / \mathrm{SrTiO}_{3}$ heterointerface", Nature 427, 6973 (2004).

${ }^{3}$ N. Reyren, S. Thiel, A. Caviglia, L. F. Kourkoutis, G. Hammerl, C. Richter, C. Schneider, T. Kopp, A.-S. Rüetschi, D. Jaccard, M. Gabay, D. A. Muller, J.-M. Triscone, and J. Mannhart, "Superconducting interfaces between insulating oxides", Science 317, 1196 (2007).

${ }^{4}$ R. Eason, ed., Pulsed laser deposition of thin films (John Wiley \& Sons, Inc., 2007).

${ }^{5} \mathrm{X}$. Fa-Xian and Z. Tong-Tong, "Topological insulator nanostructures and devices", Chinese Physics B 22, 096104 (2013).

${ }^{6}$ Y. Ando, "Topological insulator materials", Journal of the Physical Society of Japan 82, 102001 (2013).

${ }^{7}$ J. Moore, "Topological insulators: the next generation", Nature Physics 5, 378 (2009).

${ }^{8}$ S. Zhang, L. Yan, J. Qi, M. Zhuo, Y.-Q. Wang, R. Prasankumar, Q. Jia, and S. Picraux, "Epitaxial thin films of topological insulator Bi2 $\mathrm{Te}_{3}$ with two-dimensional weak anti-localization effect grown by pulsed laser deposition", Thin Solid Films 520, 6459 (2012).

${ }^{9}$ L. Meng, H. Meng, W. Gong, W. Liu, and Z. Zhang, "Growth and characterization of $\mathrm{Bi}_{2} \mathrm{Se}_{3}$ thin films by pulsed laser deposition using alloy target", Thin Solid Films 519, 7627 (2011).

${ }^{10}$ P. Le, K. Wu, C. Luo, and J. Leu, "Growth and characterization of topological insulator $\mathrm{Bi}_{2} \mathrm{Se}_{3}$ thin films on $\mathrm{SrTiO}_{3}$ using pulsed laser deposition", Thin Solid Films 534, 659 (2013).

${ }^{11}$ H. Jin, S. Rhim, J. Im, and A. Freeman, "Topological oxide insulator in cubic perovskite structure", Scientific Reports 3, 1651 (2013).

${ }^{12}$ G. Pollefeyt, S. Rottiers, P. Vermeir, P. Lommens, R. Hühne, K. D. Buyssera, and I. V. Driessche, "Feasibility study of the synthesis of $\mathrm{YBiO}_{3}$ thin films by aqueous chemical solution deposition as an alternative for $\mathrm{CeO}_{2}$ buffer layers in coated conductors", Journal of Materials Chemistry A 1, 3613 (2013).

${ }^{13}$ J. Zhang, Z. Zhang, Y. Zhang, C. Li, X. Sun, H. Si, Z. Peng, and Y. Li, "Preparation of novel $\mathrm{YBiO}_{3} / \mathrm{Bi}_{2} \mathrm{O}_{3}$ heterostructured composite with high visible light photocatalytic activity", Materials Express 5, 336 (2015).

${ }^{14} \mathrm{~V}$. Goldschmidt, "Die gesetze der krystallochemie", Die Naturwissenschaften 21, 477 (1926).

${ }^{15}$ K. Orsel, R. Groenen, H. Bastiaens, G. Koster, G. Rijnders, and K.-J. Boller, “Optimum oxidation state of $\mathrm{srtio}_{3}$ plasmas for stoichiometric growth of pld films identified by laser induced fluorescence", APL Materials 3, 106103 (2015). 
${ }^{16}$ K. Orsel, R. Groenen, H. Bastiaens, G. Koster, G. Rijnders, and K.-J. Boller, "Spatial and temporal mapping of al and alo during oxidation in pulsed laser ablation of $\mathrm{LaAlO}_{3}$ ", Journal of Instrumentation 8, C10021 (2013).

${ }^{17}$ S. Amoruso, C. Aruta, P. Aurino, R. Bruzzese, X. Wang, F. M. Granozio, and U. S. di Uccio, "Oxygen background gas influence on pulsed laser deposition process of $\mathrm{LaAlO}_{3}$ and $\mathrm{LaGaO}_{3}$ ", Applied Surface Science 258, 9116 (2012).

${ }^{18}$ S. Amoruso, B. Toftmann, and J. Schou, "Thermalization of a uv laser ablation plume in a background gas: from a directed to a diffusionlike flow", Physical Review E: Statistical, Nonlinear, and Soft Matter Physics 69, 056403 (2004).

${ }^{19}$ R. Kurucz and B. Bell, Atomic line data kurucz cd-rom no. 23, Cambridge, Mass.: Smithsonian Astrophysical Observatory, (1995) https://www.cfa.harvard.edu/ amp/ampdata/kurucz23/sekur.html.

${ }^{20} \mathrm{R}$. Pearse and A. Gaydon, The identification of molecular spectra, 3rd ed. (Chapman and Hall, London, 1976).

${ }^{21}$ A. Kramida, Y. Ralchenko, J. Reader, and N. A. T. (2013), Nist atomic spectra database (ver. 5.1), National Institute of Standards and Technology, (2013) http: //physics.nist.gov/asd.

${ }^{22}$ A. Sambri, S. Amoruso, X. Wang, M. Radovic', F. M. Granozio, and R. Bruzzese, "Substrate heating influence on plume propagation during pulsed laser deposition of complex oxides", Applied Physics Letters 91, 151501 (2007).

${ }^{23} \mathrm{R}$. Groenen, "Understanding thin film growth in pulsed laser deposition; plasma plume chemistry".

${ }^{24}$ C. Dutouquet and J. Hermann, "Laser-induced fluorescence probing during pulsed-laser ablation for three-dimensional number density mapping of plasma species", Journal of Physics D: Applied Physics 34, 3356-3363 (2001).

${ }^{25}$ S. Wicklein, A. Sambri, S. Amoruso, X. Wang, R. Bruzzese, A. Koehl, and R. Dittmann, "Pulsed laser ablation of complex oxides: the role of congruent ablation and preferential scattering for the film stoichiometry", Applied Physics Letters 101, 131601 (2012).

${ }^{26} \mathrm{C}$. Bell and D. Husain, "Kinetic studies of $\operatorname{Bi}\left(6^{4} \mathrm{~S}_{3 / 2}\right)$ by time-resolved atomic resonance fluorescence ii: an invesitgation of the third-order reactions $\mathrm{Bi}+\mathrm{O}_{2}$ $+\mathrm{M}$ and $\mathrm{Bi}+\mathrm{NO}+\mathrm{M}\left(\mathrm{M} \equiv \mathrm{He}, \mathrm{Nz}\right.$ and $\left.\mathrm{CF}_{4}\right)$ ", Journal of Photochemistry 24, 223 (1984).

${ }^{27}$ H. Qin, X. Zhang, D. Sun, T. Zhang, H. Jiang, H. Liu, Y. Sang, and J. Wanga, "Charge conversion and mass transfer on surface of $\mathrm{Al}_{2} \mathrm{O}_{3}$ nanoparticles in $\mathrm{Y}_{2} \mathrm{O}_{3}-\mathrm{Al}_{2} \mathrm{O}_{3}$ colloidal system", Design and understanding of solid-state and crystalline materials 15, 4335 (2013).

${ }^{28} \mathrm{X}$. Zhang, W. Jin, S. Hao, Y. Zhao, and H. Zhang, "Study of the crystal structures of new buffer materials $\mathrm{Bi}_{1-x} \mathrm{Y}_{x} \mathrm{O}_{1.5}$ ", Journal of Superconductivity and Novel Magnetism 23, 1011 (2010). 
${ }^{29}$ C. Aruta, S. Amoruso, R. Bruzzese, X. Wang, D. Maccariello, F. M. Granozio, and U. S. di Uccio, "Pulsed laser deposition of $\mathrm{SrTiO}_{3} / \mathrm{LaGaO}_{3}$ and $\mathrm{SrTiO}_{3} / \mathrm{LaAlO}_{3}$ : plasma plume effects", Applied Physics Letters 97, 252105 (2010).

${ }^{30}$ C. Xu, S. Wicklein, A. Sambri, S. Amoruso, M. Moors, and R. Dittmann, "Impact of the interplay between nonstoichiometry and kinetic energy of the plume species on the growth mode of $\mathrm{SrTiO}_{3}$ thin films", Journal of Physics D: Applied Physics 47, 034009 (2014). 


\section{CONCLUSIONS AND DISCUSSION}

The goal of the work presented in this thesis is to realize a spectroscopic spatiotemporal mapping of selected species in a specific transient plasma for a better understanding of the internal plasma dynamics and chemistry. The type of plasma under investigation, a laser-induced plasma for pulsed laser deposition (PLD), has been selected in view of its significant relevance for material science. Especially unravelling the detailed influence of global external parameters in PLD, such as the fluence of the ablation laser and the composition of the background gas and its pressure, on the plasma dynamics and chemistry are of interest, because this can be the key to an improved understanding and control of stoichiometric film growth.

The spectroscopic approach that we have chosen is a combination of laserinduced fluorescence (LIF) and absorption spectroscopy (AS). LIF enables the detection of the constituents even in "dark" plasmas, i.e., also when the plasma plume has reached the substrate, where it has cooled down and no longer spontaneously fluoresces. AS provides a means to calibrate the relative density distribution maps generated by LIF measurements for obtaining absolute density distributions.

This research was carried out in parallel and in close collaboration with a second set of investigations performed by a colleague $\mathrm{PhD}$ student (Rik Groenen) in the IMS group (UT) who focused his PLD work on the morphology of the grown films. In that part of the work, the properties of grown films are investigated, both during deposition with RHEED, as well as after film growth with X-ray diffraction measurements and atomic force microscopy.

The combination of spectroscopic (also called chemical) in-situ spatiotemporal mapping of individual plasma constituents with a detailed analysis of the grown films has enabled an unparalleled insight in the influence of the external PLD parameters on the plasma propagation, chemical evolution and material deposition. Having investigated different types of plasmas for growing different types of perovskites has shown that the choice of materials and background gas is of decisive influence. 
The first material we chose to investigate was $\mathrm{LaAlO}_{3}$ (LAO) motivated by one of its interesting but only incompletely understood growth features. Specifically, when LAO is grown on a substrate of $\mathrm{SrTiO}_{3}$ (STO) under certain conditions, a thin film oxide heterostructure is formed, which exhibits a high concentration of charge carriers with high mobility confined to the interface of LAO and STO, also referred to as a two-dimensional electron gas (2DEG). One of the conditions for the formation of this 2DEG was found to be film growth at low oxygen pressures $\left(10^{-6}\right.$ mbar). A possible explanation is that, at these pressures, the LAO film extracts oxygen from the STO, creating conducting vacancies. We mapped the oxidation of aluminium spatially and temporally in an oxygen background of $1 \cdot 10^{-1}$ mbar. Oxidation of $\mathrm{Al}$ in the front of the plume has been observed, where direct mixing of the plasma with the background gas takes place. Oxidation of ground state $\mathrm{Al}$ also takes place in the center of the plume, but at a lower rate. An increase in $\mathrm{Al}$ density together with the absence of $\mathrm{AlO}$ in a pure Ar background (oxygen absent) confirms that aluminium is indeed oxidizing. The absence of $\mathrm{Al}$ in the front of the plasma plume in $\mathrm{O}_{2}$ compared to Ar, which was visible at various different oxygen pressures $\left(1 \cdot 10^{-1}, 1 \cdot 10^{-2}\right.$ and $\left.1 \cdot 10^{-3} \mathrm{mbar}\right)$, suggest that oxidation still takes place at these settings, but at a lower rate. This would support the observations of increased oxygen vacancies in the boundary layer between STO and LAO at lower background pressures.

For the next step of investigations, we chose for a material/substrate combination that might provide more fundamental insight in the growth process through minimum complexity, such as growing a stoichiometric film on a crystalline substrate of the very same material. Although this might appear less interesting due to its apparent predictability, a remarkably strong influence is found of the external parameters of PLD, such as the ablation laser fluence and background gas composition, on the stoichiometry of the film growth. As a deposition material, we chose STO, as this is a well-known and well-investigated PLD reference system. As such, much research has already been done as to quantifying the heuristic influence of the external PLD parameters on the film growth. However, much of this research treats the PLD plasma as a "black box", in that it only investigates the influence of the global input parameters on the output, which in this case is the grown film. Especially in chemically reactive plasmas, a direct link to the exact molecular state in which material arrives at the substrate can be the key to a deeper understanding of growing perfect crystalline thin films but is difficult to gain via blind optimization of PLD parameters. Using an STO plasma, we have mapped dynamic oxidation processes of plasma species and have shown the strong influence of the composition of the background gas on the plasma plume composition. Comparing these spectroscopic data with X-ray diffraction data of the grown material quality, we find a clear correlation between the chemical composition of the leading edge of the plasma plume and the stoichiometry of the grown film. Above a specific $\mathrm{O}_{2}$ fraction of the background gas, approximately $75 \%$, when full oxidation of $\mathrm{Ti}$ and $\mathrm{Sr}$ in the leading edge of the plasma plume is reached, the film growth transitions from 
non-stoichiometric to stoichiometric growth, as determined with X-Ray diffraction. This suggests that the oxidation of species of the plasma-before the constituents reach the substrate-is a crucial mechanism in the stoichiometric reconstruction of the synthesized oxide thin films.

For the third material, we chose a much less understood type of bismuth-based perovskite that has piqued interest lately, but which has only been grown a few times using PLD and which also appears problematic with regards to epitaxial growth. The material chosen, $\mathrm{YBiO}_{3}(\mathrm{YBO})$, has recently been predicted to be a topological insulator (TI), a new class of materials with unique quantum-mechanical properties, as they present time-reversal-symmetry-protected surface states while having electrically insulating bulk states. A major difficulty for growing YBO may originate from the unfavorable ratio of ionic radii of the cations, which might have prevented the straightforward growth of bulk YBO and single crystalline films with PLD. A secondary problem with the stoichiometric growth of YBO in PLD is found in the volatility of $\mathrm{Bi}$, which hardly oxidizes. This would result in a low sticking factor, causing little $\mathrm{Bi}$ to be available for synthesizing a stoichiometric YBO film. With our research on YBO plasma and film growth, we try to better clarify the actual influence of these factors on the much reduced reliability and predictability of appropriate growth parameters for PLD yielding stoichiometric YBO.

We have mapped the dynamic oxidation and propagation processes of $\mathrm{Y}$ and $\mathrm{Bi}$ in the plasma plume during PLD of YBO, and find that little direct chemical interaction appears to take place between the plasma plume constituents and the background gas. However, a strong influence of the background gas composition can be seen on the plasma composition, which appears not to be caused by a mixing and reaction of the plasma plume with the background gas, but by reactions between the background gas and the substrate. X-ray diffraction data of the grown material quality shows a strong increase of the YBO nucleation rate with an increase in $\mathrm{O}_{2}$ background fraction. Below $20 \% \mathrm{O}_{2}$, only a small amount of poly-crystalline $\mathrm{Y}_{2} \mathrm{O}_{3}$ is deposited. At $20 \%$ oxygen, epitaxial $\mathrm{YBO}$ grows, and around $80 \% \mathrm{O}_{2}$, the nucleation rate has increase enough for a non-epitaxial orientation of YBO to also appear. Remarkably, these transitions in film growth do not coincide with a significant change in plasma composition, as we find that the plasma composition only slightly changes with background gas composition. This has led us to conclude that the strong influence of the background gas composition seen in the XRD measurements is on the actual synthesis of YBO film from the plasma constituents, not on the plasma plume composition. $\mathrm{O}_{2}$ from the background gas appears to be a crucial building block for forming, at the surface of the substrate, $\mathrm{YBiO}_{3}$ from the $\mathrm{Bi}$ and YO provided by the plasma plume.

From the three materials we have investigated, it can already be seen that spatiotemporally resolved LIF is a highly valuable analytic instrument for understanding PLD growth processes. Even when only mapping a select number of plasma constituents of a deposition material, much additional insight can be obtained. Having applied LIF in various different material systems, here, LAO on STO, STO 
on STO, and YBO on LSAT, has clearly shown that there are no universal plasma and growth dynamics. Instead, each material system, due to its individual chemical and physical properties, provides its own dynamics in the plasma, both at the substrate and at the target. This observed wide span in variety promises that there is much more to discover and understand through LIF and possibly also other types of spectroscopy. Also, it might be attractive to consider LIF as an additional standard monitoring tool, e.g., for achieving additional control in growth or for upscaling PLD growth in speed or area.

During our research, we have also come across many interesting issues for future research and actions for improvement.

- A strong influence on the plasma plume propagation velocity can be seen in spontaneous emission measurements when using a resistively heated substrate, as compared to plasma propagation with the substrate at room temperature. A question that remains open is whether the substrate temperature only influence the propagation velocity, or whether it also changes the plume composition or oxidation processes. A mapping of, for instance, $\mathrm{Al}$ and $\mathrm{AlO}$ with a substrate in place at room temperature and at $700^{\circ} \mathrm{C}$ would allow for insight in this question.

- During our measurements of YBO (chapter 6) we found that much of the atomic Bi remained near the ablation target, whereas one typically observes most other materials propagating towards the substrate. Related to this observation might be that a significant increase in growth speed per ablation pulse of YBO films is observed when the target is ablated in a so-called burst mode (a one-second-burst of pulses at $50 \mathrm{~Hz}$ ) as compared to a constant ablation rate of $2 \mathrm{~Hz}$. A possible explanation for faster growth per pulse in burst mode could be that with an increased repetition rate the pulses propel part of the Bi that lingers close to the target from previous pulses and thus provide a higher concentration of $\mathrm{Bi}$ in the front of the plume. A measurement of the Bi density directly after the first ablation pulse of a $50 \mathrm{~Hz}$ burst, followed by a measurement of the Bi density at a later time during the burst might provide an answer.

- In none of our experiments have we observed the so-called snowplough effect reported by others in measurements with high background gas pressures ( $>0.1$ mbar). The explanation given for this effect is that during plasma propagation in a background gas, a significant increase in density occurs in the leading edge of the plasma plume due to collisions with the background gas. Most of these measurements are carried out using an iCCD camera, which spectrally integrates images over its entire spectral sensitivity range. We wonder whether a more appropriate interpretation of the observations is possible via the chemically-specific nature of our measurements. An apparent increase in measured intensity in certain regions of a plasma plume could for instance be caused by a shift of emission wavelength due to chemical reactions taking place here. If an oxidized species emits light at a wavelength 
for which the iCCD camera is much more sensitive compared to the wavelength emitted by the corresponding atomic species, this would give rise to an increase of the output signal of the camera in this region. We have observed an increase in oxidized molecular species in the outer edges of the plasma plume, which can lead to an increase or a spectral shift of spontaneous emission in these regions.

Regarding the instrumentation, several improvements and additions can be considered for future research.

- The sensitivity of the resonance fluorescence measurements (i.e. detection of fluorescence at the same wavelength as the excitation wavelength) can still be improved significantly by further reducing scattering of the probe laser at various inner surfaces of the PLD chamber. Particularly, coating the inner surface of the PLD chamber and the tubing and flanges of LIF excitation laser ports with a light-absorbing material would significantly reduce probe beam scattering.

- The range of dyes that can be used in the dye laser can be extended to also include the shorter wavelengths in the visible and near UV. This would give access to the wavelengths in the gap between the direct output of the current dye laser and its second harmonic, allowing for the excitation of a larger variety of species or transitions. Furthermore, the frequency doubled output of from blue and UV dyes towards the range of 250 to $200 \mathrm{~nm}$ would open up the possibility to excite LIF via two-photon absorption (TALIF). This would allow the investigation of densities of background gasses such as Ar, as well as measurements of atomic oxygen in the plasma plume. Tracking an inert gas allows for investigating the influx of the background gas into the propagating plasma plume. Especially mapping of atomic oxygen is of particular interest, as very little is known about the behavior of oxygen ablated from a perovskite target. 


\section{List of Publications}

\section{Papers}

- K. Orsel, H.M.J. Bastiaens, R. Groenen, G. Koster, A.J.H.M. Rijnders, K.-J. Boller, Laser-induced fluorescence alanysis of plasmas for stoichiometric growth of $\mathrm{YBiO}_{3}$ films with pulsed laser deposition, In preparation

- K. Orsel, H.M.J. Bastiaens, R. Groenen, G. Koster, A.J.H.M. Rijnders, K.-J. Boller, Influence of the oxidation state of $\mathrm{SrTiO}_{3}$ plasmas for stoichiometric growth of pulsed laser deposition films identified by laser induced fluorescence, APL Materials, vol. 3, pp. 106103, 2015

- R. Groenen, J. Smit, K. Orsel, A. Vailionis, H.M.J. Bastiaens, M. Huijben, K.-J. Boller, A.J.H.M. Rijnders, G. Koster, Stoichiometry controlled oxide thin film growth by pulsed laser deposition, APL Materials, vol. 3, pp. 070701, 2015

- K. Orsel, H.M.J. Bastiaens, R. Groenen, G. Koster, A.J.H.M. Rijnders, K.-J. Boller, Temporal and spatial mapping of oxidized species in pulsed laser deposition plasmas, Journal of Instrumentation, vol. 8, pp. C10021, 2013

\section{Oral presentations}

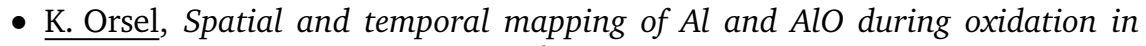
pulsed laser ablation of $\mathrm{LaAlO}_{3}, 37^{\text {th }}$ Annual Meeting NNV Division of Atomic, Molecular and Optical Physics, Lunteren, The Netherlands (1 and 2 October 2013)

- K. Orsel, Spatial and temporal mapping of Al and AlO during oxidation in pulsed laser ablation of $\mathrm{LaAlO}_{3}, 16^{\text {th }}$ Laser Aided Plasma Diagnostics Symposium, Madison, United States (23 - 26 September 2013)

- K. Orsel, Applied laser spectroscopy for plasmas, MESA+ Annual Meeting, Enschede, The Netherlands (16 September 2013)

- K. Orsel, Spatial and temporal mapping of plasma species, ASML Meeting at LPNO, Enschede, The Netherlands (17 April 2013) 


\section{Poster presentations}

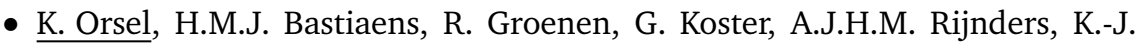
Boller, Transient composition changes in a pulsed laser ablation plasma, $38^{\text {th }}$ Annual Meeting NNV Division of Atomic, Molecular and Optical Physics, Lunteren, The Netherlands (7 and 8 October 2014)

- K. Orsel, H.M.J. Bastiaens, R. Groenen, G. Koster, K. Beks-Peerenboom, J. van Dijk, A.J.H.M. Rijnders, K.-J. Boller, Oxidation and stoichiometry studies of pulsed laser ablation plasmas, $26^{\text {th }}$ Symposium Plasma Physics \& Radiation Technology, Lunteren, The Netherlands (11 and 12 March 2014)

- K. Orsel, H.M.J. Bastiaens, R. Groenen, G. Koster, K. Beks-Peerenboom, J. van Dijk, A.J.H.M. Rijnders, K.-J. Boller, Spatial and temporal evolution of PLD plasmas, $25^{\text {th }}$ Symposium Plasma Physics \& Radiation Technology, Lunteren, The Netherlands (5 and 6 March 2013)

- R. Groenen, G. Koster, D.H.A. Blank, A.J.H.M. Rijnders, K. Orsel, H.M.J. Bastiaens, K.-J. Boller, The influence of laser parameters in Pulsed Laser Deposition on thin film growth, $25^{\text {th }}$ Symposium Plasma Physics \& Radiation Technology, Lunteren, The Netherlands (5 and 6 March 2013)

- K. Orsel, H.M.J. Bastiaens, R. Groenen, G. Koster, K. Beks-Peerenboom, J. van Dijk, A.J.H.M. Rijnders, K.-J. Boller, Laser Induced Fluorescence (LIF) in laser produced plasmas, Physics@FOM, Veldhoven, The Netherlands (22 and 23 January 2013)

- K. Orsel, H.M.J. Bastiaens, R. Groenen, G. Koster, K. Beks-Peerenboom, J. van Dijk, A.J.H.M. Rijnders, K.-J. Boller, Laser Induced Fluorescence (LIF) in laser produced plasmas, $36^{\text {th }}$ Annual Meeting NNV Division of Atomic, Molecular and Optical Physics, Lunteren, The Netherlands (9 and 10 October 2012)

- K. Orsel, H.M.J. Bastiaens, R. Groenen, G. Koster, K. Beks-Peerenboom, J. van Dijk, A.J.H.M. Rijnders, K.-J. Boller, Laser Induced Fluorescence (LIF) in laser produced plasmas, MESA+ Annual Meeting, Enschede, The Netherlands (18 September 2012)

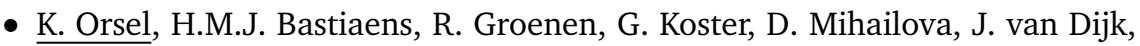
A.J.H.M. Rijnders, K.-J. Boller, In-situ mapping of $3 D$ material flux in PLD plasmas, $24^{\text {th }}$ Symposium Plasma Physics \& Radiation Technology, Lunteren, The Netherlands (6 and 7 March 2012)

- R. Groenen, G. Koster, D.H.A. Blank, A.J.H.M. Rijnders, K. Orsel, H.M.J. Bastiaens, K.-J. Boller, The influence of laser parameters in Pulsed Laser Deposition on thin film growth, $24^{\text {th }}$ Symposium Plasma Physics \& Radiation Technology, Lunteren, The Netherlands (6 and 7 March 2012) 
- K. Orsel, H.M.J. Bastiaens, R. Groenen, G. Koster, D. Mihailova, J. van Dijk, A.J.H.M. Rijnders, K.-J. Boller, Large-Area Pulsed Laser Deposition of thin films with atomic precision, $35^{\text {th }}$ Annual Meeting NNV Division of Atomic, Molecular and Optical Physics, Lunteren, The Netherlands (11 and 12 oktober 2011)

- K. Orsel, H.M.J. Bastiaens, R. Groenen, G. Koster, J. van Dijk, A.J.H.M. Rijnders, K.-J. Boller, Large-Area Pulsed Laser Deposition of thin films with atomic precision, Ph.D. Network Workshop "Materials for Energy", Hollum, The Netherlands (19 - 24 June 2011)

- R. Groenen, G. Koster, D.H.A. Blank, A.J.H.M. Rijnders, K. Orsel, H.M.J. Bastiaens, K.-J. Boller, The influence of laser parameters in Pulsed Laser Deposition (PLD) on kinetic and stoichiometric growth characteristics, Ph.D. Network Workshop "Materials for Energy", Hollum, The Netherlands (19 - 24 June 2011)

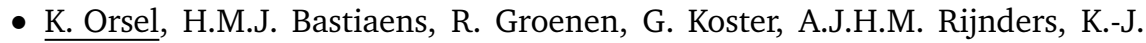
Boller, Large Area Pulsed Laser Deposition, $23^{\text {rd }}$ Symposium Plasma Physics \& Radiation Technology, Lunteren, The Netherlands (15 and 16 March 2011)

- R. Groenen, G. Koster, D.H.A. Blank, A.J.H.M. Rijnders, K. Orsel, H.M.J. Bastiaens, K.-J. Boller, The influence of laser parameters in Pulsed Laser Deposition (PLD) on kinetic and stoichiometric growth characteristics, $23^{\text {rd }}$ Symposium Plasma Physics \& Radiation Technology, Lunteren, The Netherlands (15 and 16 March 2011)

- R. Groenen, G. Koster, D.H.A. Blank, A.J.H.M. Rijnders, K. Orsel, H.M.J. Bastiaens, K.-J. Boller, The influence of laser parameters in Pulsed Laser Deposition on thin film growth, MAMA conference, Ercolano, Italy (15 and 16 March 2011)

\section{Conference proceedings}

- K. Orsel, H.M.J. Bastiaens, R. Groenen, G. Koster, A.J.H.M. Rijnders, K.-J. Boller, Temporal and spatial mapping of oxidized species in pulsed laser deposition plasmas, Journal of Intrumentation, vol. 8, pp. C10021, 2013

\section{Other publications}

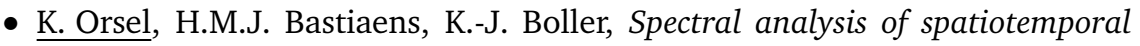
and chemical dynamics in transient laser plasmas, Research highlight in the MESA+ Annual report 2013 


\section{ACKNOWLEDGMENTS}

Before you lies my PhD thesis, the culmination of five years of work. A PhD research project is rarely easy, but fortunately, I could always count on the support of many people in the past years, whether it was in the form of scientific assistance whenever I got stuck, or moral support when I needed a short break, or simply a push in the back.

First of all I want to thank my professor and promoter Klaus Boller for giving me the opportunity to do this research and accepting me into the LPNO group. Your vast knowledge of seemingly everything optics and ability to explain any of it with just a pen and piece of paper will never cease to amaze me.

My supervisor, Bert Bastiaens, also deserves my gratitude. Despite how busy you often were, the open door to your office was always inviting. Your tendency to always criticize and deliberately misinterpret anything I wrote could be frustrating at times, but in the end, it always improved it and has tremendously improved my scientific writing skills. Our many discussions have helped me to grow, both as a scientist and on a personal level.

Everyone at the Laser Physics and Nonlinear Optics group deserves thanks for many fruitful discussions and a great working atmosphere over the past years. The PhD-dinners, the Wednesday-meeting-jokes, interesting discussions at the coffee table, Friday-afternoon-drinks and group retreats made my time spent on this research project highly enjoyable. So thank you: Ab, Ale, Carin, CaterinA, CaterinB, Chris, Edith, Hannah, Ingrid, Jean, Jesse, Jörn, Joanna, Jonathan, Marco, Mathijs, Muharrem, Peter, Reinier, Robert, Ron, Ruud, Simone, Thomas and Youwen.

My paranymphs Tom and Yin deserve special thanks. Tom was always willing to help me, whether it was with something scientific, with getting coffee for the group, or with some crazy idea for a Friday-afternoon-experiment. Yin and I started at the same time, we went to many of the same conferences and we both worked on setups that, to put it mildly, were technically challenging. I also want to thank Gerard and Ramon, without who's technical expertise and experience the resurrection of the $\mathrm{XeCl}$ excimer laser would never have been possible.

I want to thank the members of the Inorganic Materials Science group. In particular their professor, Guus Rijnders, who is one of my promoters, and Gertjan Koster, who's knowledge of PLD and thin films was very helpful. Rik Groenen 
worked on a $\mathrm{PhD}$ project parallel and connected to mine. I am grateful for our discussions that greatly helped me understand the PLD and thin-film parts of my project, and for allowing me to use parts of his research in my papers and this thesis.

I also want to extend my gratitude to the members of the Complex Photonic Systems group and the Optical Sciences group, for the use of their facilities and the lending of optical equipment.

Aside from my colleagues, I also have many friends who I want to thank for making my spare time much more interesting and fun. The UT swim group "De vroege vogels", where I greatly improved my swimming skills and became addicted to the sport, even though it was always too early in the morning and the outdoor pool at times came close to answering the question "What does hypothermia feel like?". Musilon, Twents Studentenkoor, and especially my barbershop quintet "Het overmatig kwartet" (Henk, Huub, Nico and Pim), for many hours of singing and fun. My gaming groups, for many enjoyable evenings, and in particular Danny, Kevin and Oboema, for DM'ing fantastic D\&D games.

Finally, I would like to thank my family, who have always supported me in everything I have done, and were always willing to lend me a helping hand or listening ear whenever needed. 
Cover: Stylized photo of laser-induced fluorescence of yttrium in a plasma generated by ablating an yttria target. The picture is taken inplane with the excitation light sheet, which was tuned to an excitation wavelength of $Y$ at $294.85 \mathrm{~nm}$ (invisible in this picture). The excitation by the UV light results in fluorescence at several wavelengths between 535 and $565 \mathrm{~nm}$, visible in this picture as the thin green line through the plasma.

ISBN: $978-90-365-4052-0$ 